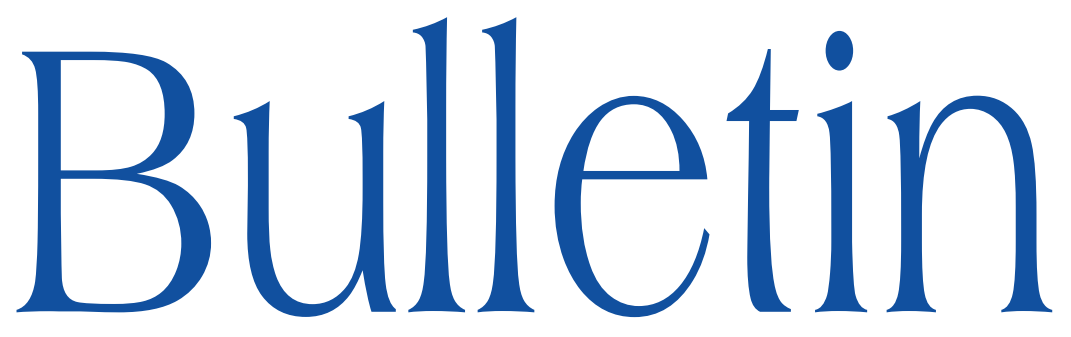

de la SOCIÉTÉ MATHÉMATIQUE DE FRANCE

\title{
SINGULARITÉS À L'INFINI ET INTÉGRATION MOTIVIQUE
}

\author{
Michel Raibaut
}

Tome 140

Fascicule 1

2012 


\title{
SINGULARITÉS À L'INFINI ET INTÉGRATION MOTIVIQUE
}

\author{
PAR MICHEL RAIBAUT
}

RÉsumé. - Soit $k$ un corps de caractéristique nulle et $f$ une fonction non constante définie sur une variété lisse. Nous définissons dans cet article une fibre de Milnor motivique à l'infini qui appartient à un anneau de Grothendieck des variétés. Elle est définie en termes d'une compactification choisie, non nécessairement lisse, mais est indépendante de ce choix. Lorsque $k$ est le corps des nombres complexes, en utilisant le morphisme de réalisation de Hodge, elle se réalise en le spectre à l'infini de $f$. Nous la calculons par exemple, dans le cas d'un polynôme non dégénéré pour son polyèdre de Newton à l'infini.

Pour toute valeur $a$, nous définissons une fibre de Milnor motivique complète $S_{f, a}$ qui prolonge la fibre de Milnor motivique usuelle $S_{f-a}$. Ceci permet d'introduire des valeurs motiviquement atypiques, un ensemble de bifurcation motivique de $f$ et une notion de fonction motiviquement modérée.

Abstract (Singularities at infinity and motivic integration). - Let $k$ be a field of characteristic zero and $f$ be a non constant function defined on a smooth variety. We construct in this article a motivic Milnor fiber at infinity which belongs to a Grothendieck ring of varieties. It is defined in terms of a chosen compactification, not necessary smooth, but is shown to be independent of this choice. When $k$ is the field of complex numbers, using the Hodge realization morphism, it specializes to the

Texte reçu le 29 novembre 2010, accepté le 29 avril 2011.

Michel Raibaut, Laboratoire Jean-Alexandre Dieudonné, Université de Nice, Parc Valrose, 06108 NICE Cedex 2, France and University catholic of Leuven, Department of Mathematics, Celestijnenlaan 200 B,B-3001 Leuven (Heverlee), Belgium E-mail : raibaut@unice.fr

Ce travail a bénéficié d'une aide des Agences Nationales de la Recherche portant les références ANR-08-JCJC-0118-01, et ANR-06-BLAN-0183, d'une aide du Projet de Recherche G.0318.06N de la Fondation de Recherche - Flandre (FWO) et d'une aide du projet ERC Advanced Grant NMNAG. 
spectrum at infinity of $f$. As an example, we compute it in the case of a Laurent polynomial non-degenerated with respect to its Newton polyhedron at infinity.

For each value $a$, we define a complete motivic Milnor fiber $S_{f, a}$. This object is an extension of the usual motivic Milnor fiber $S_{f-a}$. Then we introduce motivic atypical values, a motivic bifurcation set of $f$ and a notion of motivically tame function.

\section{Introduction}

Soit $U$ une variété algébrique complexe lisse et $f: U \rightarrow \mathbb{A}_{\mathbb{C}}^{1}$ une application régulière non constante. Il existe un ensemble fini minimal $\mathscr{B}$, appelé ensemble de bifurcation tel que $f: U \backslash f^{-1}(\mathscr{B}) \rightarrow \mathbb{A}_{\mathbb{C}}^{1} \backslash \mathcal{B}$ soit une fibration topologique localement triviale. L'ensemble des valeurs critiques de $f$ est contenu dans l'ensemble de bifurcation, mais l'inclusion peut être stricte. Par exemple le polynôme $x(x y-1)$ considéré par Broughton [4] est une fonction lisse sur $\mathbb{C}^{2}$ et toutes ses fibres sont connexes exceptée celle en 0 . Les valeurs de bifurcation non critiques sont dues à des «singularités à l'infini ». Hà et Lê [45] donnent une description complète de l'ensemble de bifurcation dans le cas des courbes. Précisément, une valeur $a$ est de bifurcation si et seulement si la caractéristique d'Euler de sa fibre diffère de la caractéristique d'Euler de la fibre générique. En dimension quelconque le problème est ouvert, voir Broughton [4], CassouNoguès et Dimca [6], Némethi et Zaharia [28], Parusiński [30], Tibăr [41] et [42], et Zaharia [46].

De nombreuses définitions de bon comportement à l'infini ont été introduites pour les fonctions polynomiales. Broughton [4] définit les polynômes modérés, généralisés ensuite par Némethi et Zaharia [29] en les polynômes $M$-modérés, puis en les polynômes sans singularités à l'infini par Siersma et Tibăr [39]. Pour une large classe de polynômes contenant ceux cités ci-dessus, il existe une analogie forte entre les propriétés des singularités isolées et les propriétés de ces polynômes. La fibre générique de la fibration a par exemple, comme pour les singularités isolées, le type d'homotopie d'un bouquet de sphères de dimension $\operatorname{dim} U-1$. Parusiński [31] et Sabbah [36] ont enfin introduit la classe des polynômes cohomologiquement modérés pour traîter des questions cohomologiques sur $\mathbb{Q}$. Les différentes classes introduites ci-dessus ne se contiennent pas entre elles et on appelle polynôme faiblement modéré l'un de leurs éléments [27].

Les espaces de cohomologie à support compact $H_{c}^{*}\left(f^{-1}(t), \mathbb{Q}\right)$ de la fibre en $t$ sont munis d'une structure de Hodge mixte naturelle [8]. Steenbrink et Zucker [40] puis M. Saito [38] ont montré comment construire une structure de Hodge mixte limite lorsque $t$ tend vers l'infini. Sabbah [35] l'a retrouvée en considérant la transformation de Fourier sur des modules convenables sur

TOME $140-2012-\mathrm{N}^{\mathrm{O}} 1$ 
l'anneau des opérateurs différentiels. En particulier si $(X, i, \hat{f})$ est une compactification lisse de $f$, et $1 / \hat{f}$ prolonge $1 / f$, la structure de Hodge mixte limite sur $H_{c}^{k}\left(f^{-1}(t), \mathbb{Q}\right)$ s'identifie alors à la structure de Hodge mixte du $i$-ème groupe d'hypercohomologie à support compact $\mathbb{H}_{c}^{k}\left(\hat{f}^{-1}(\infty), \psi_{1 / \hat{f}}\left(R i_{!} \underline{\mathbb{Q}}_{U}\right)\right)$ de la fibre à l'infini, à coefficients dans le faisceau des cycles proches de $1 / \hat{f}$ évalué sur l'extension par $0 \mathrm{du}$ faisceau constant sur $U$. On pourra consulter [35, (5.5)]. Le spectre de cette structure limite est un invariant du polynôme $f$ appelé spectre à l'infini, il a notamment été étudié (souvent pour la cohomologie sans support) par Brélivet [2] et [3], Dimca [12], García-López et Némethi [14], [16] et [15], Némethi et Sabbah [27] et Sabbah [35] et [37].

Considérons maintenant une fonction $g: X \rightarrow \mathbb{A}^{1}$ définie sur une variété algébrique lisse. Soit $x$ un point fermé de $g^{-1}(0)$. En utilisant l'intégration motivique, Denef et Loeser [9] [11] introduisent la fibre de Milnor motivique $S_{g, x}$. Cet objet appartient à un anneau de Grothendieck des variétés virtuelles munies d'une action du groupe multiplicatif $\mathbb{G}_{m}$. Il se réalise, dans l'anneau de Grothendieck des structures de Hodge munies d'un endomorphisme quasiunipotent noté $K_{0}\left(S H^{\text {mon }}\right)$, en la classe de la structure de Hodge mixte limite de la fibre de Milnor de $g$ en $x$. Il permet en particulier de retrouver le spectre de Hodge-Steenbrink $\operatorname{Sp}(g, x)$ [9].

Pour toute immersion ouverte $i: U \rightarrow X$, Bittner [1] grâce au théorème de factorisation faible, puis Guibert, Loeser et Merle [19] à l'aide d'arcs raisonnablement tangents au fermé complémentaire, construisent $S_{g, U}$, un analogue motivique du faisceau $\Psi_{g}\left(R i_{!} \mathbb{Q}_{U}\right)$. Ils étendent ainsi la fibre de Milnor motivique en un morphisme additif défini sur un anneau de Grothendieck des variétés relatives $Y \rightarrow X$. Dans la partie 1, nous rappelerons cela et nous fixerons les notations utilisées par la suite.

Ainsi, pour un morphisme $f: U \rightarrow \mathbb{A}_{\mathbb{C}}^{1}$ à source lisse, en considérant $U$ comme ouvert dans une compactification, nous définissons dans la partie 2 une fibre de Milnor motivique à l'infini $S_{f, \infty}$. Cet objet est un nouvel invariant de $f$ qui ne dépend d'aucune compactification et se réalise dans l'anneau $K_{0}\left(S H^{\mathrm{mon}}\right)$ en la classe de la structure de Hodge mixte à l'infini. En particulier cette variété virtuelle munie d'une action de $\mathbb{G}_{m}$ permet de retrouver le spectre à l'infini de $f$.

Pour une fonction polynomiale $f$ s'annulant en 0 et non dégénérée pour son polyèdre de Newton $\Gamma$ en 0 , Guibert [17] calcule la fibre de Milnor motivique de $f$ au point 0 , en termes du polyèdre $\Gamma$. Ce calcul se fait sans résolution des singularités et est valable sur tout corps de caractéristique 0. Dans la partie 3, pour $f$ un polynôme non dégénéré pour son polyèdre de Newton à l'infini $\Gamma$, nous calculons de manière similaire, la fibre de Milnor motivique de $f$ à l'infini, en termes de $\Gamma$. Nous obtenons ainsi, une décomposition du spectre à l'infini 
de $f$ en termes de spectres de variétés quasi-homogènes. Ces résultats étaient annoncés dans [34].

Enfin dans la partie 4, nous définissons pour toute valeur a, une fibre de Milnor motivique «complète » $S_{f, a}$ qui ne dépend pas de la compactification et qui prolonge la fibre de Milnor motivique classique $S_{f-a}$. En particulier dans le cas d'un polynôme homogène, $S_{f, 0}$ et $S_{f, \infty}$ s'échangent par une inversion. Ceci nous permet de définir des cycles évanescents motiviques à l'infini et un ensemble de bifurcation motivique contenu dans un discriminant universel, obtenu en considérant toutes les compactifications et leurs log-résolutions. En particulier, nous dirons qu'une fonction est motiviquement modérée si et seulement si elle n'a pas de cycles évanescents motiviques à l'infini. Cette propriété est un analogue motivique de la notion de polynôme cohomologiquement modéré. Dans ce cas là l'ensemble de bifurcation motivique est égal au discriminant du polynôme. Nous montrons par exemple, qu'un polynôme commode et non dégénéré pour son polyèdre de Newton à l'infini est motiviquement modéré.

Signalons les travaux en cours de Matsui-Takeuchi [26] et [25]. Les auteurs introduisent une fibre de Milnor motivique à l'infini à l'aide de résolutions des singularités, sans prouver toutefois l'indépendance de l'objet vis à vis de la compactification. Dans le cas d'un polynôme non dégénéré pour son polyèdre de Newton à l'infini, ils décrivent les blocs de Jordan de la monodromie à l'infini en termes du polyèdre de Newton.

L'auteur tient à remercier les professeurs Johannes Nicaise et Wim Veys pour leur invitation pendant l'automne 2010 au département de mathématiques de l'université de Leuven où cet article a été rédigé.

L'auteur tient particulièrement à remercier Michel Merle, pour l'avoir intéressé aux questions ci-dessus, pour ses conseils et enfin pour toute l'attention qu'il a portée à ce travail au cours de sa réalisation.

L'auteur témoigne enfin toute sa gratitude au referee pour ses suggestions qui lui ont permis d'améliorer ce texte.

\section{Fibre de Milnor motivique}

Dans cette partie nous présentons les outils utilisés par la suite. On pourra se référer aux articles [11], [23], [24] puis [19] et [18].

\subsection{Anneaux de Grothendieck}

1.1.1. Variétés. - Dans tout ce qui suit, $k$ est un corps de caractéristique 0 et $\mathbb{G}_{m}$ est son groupe multiplicatif. On appelle $k$-variété, un schéma séparé réduit de type fini sur $k$. Si $X$ est un schéma, on notera $X_{\text {red }}$ le schéma réduit associé. On note $\operatorname{Var}_{k}$ la catégorie des $k$-variétés et pour une $k$-variété $S$ on note $\operatorname{Var}_{S}$ la catégorie des $S$-variétés, c'est à dire des morphismes $X \rightarrow S$ dans $\operatorname{Var}_{k}$. Soit 
$G$ un groupe algébrique, et $X$ une variété sur laquelle $G$ agit. L'action est dite bonne, si toute $G$-orbite est contenue dans un ouvert affine de $X$.

Soit $X$ une variété sur $k$, et $p: A \rightarrow X$ un fibré affine pour la topologie de Zariski. Les fibres de $p$ sont des espaces affines et les morphismes de transition entre les cartes sont des applications affines. Soit $G$ un groupe algébrique linéaire. Une bonne action de $G$ sur $A$ est dite affine si et seulement si elle relève une bonne action de $G$ sur $X$ et sa restriction à toutes les fibres est affine. Dans toute la suite nous supposerons les actions bonnes.

1.1.2. Anneaux de Grothendieck. - Soit $S$ une $k$-variété munie de l'action triviale de $\mathbb{G}_{m}$ et $X$ une $S$-variété munie d'une bonne action de $\mathbb{G}_{m}$ notée $\sigma$. Un morphisme $\pi: X \rightarrow \mathbb{G}_{m}$ est dit homogène de poids $n$, si $\pi(\sigma(\lambda, x))$ est égal à $\lambda^{n} \pi(x)$ pour tout $\lambda$ dans $\mathbb{G}_{m}$ et pour tout $x$ dans $X$. Pour un entier $n$ strictement positif, notons $\operatorname{Var}_{S \times \mathbb{G}_{m}}^{\mathbb{G}_{m}, n}$ la catégorie des variétés relatives $X \rightarrow S \times \mathbb{G}_{m}$ munies d'une bonne action de $\mathbb{G}_{m}$, telle que la projection sur $S$ soient $\mathbb{G}_{m}$-équivariante et la projection sur $\mathbb{G}_{m}$ soit homogène de poids $n$.

DÉfinition 1.1. - Pour tout entier $n$ strictement positif, l'anneau de Grothendieck noté $K_{0}\left(\operatorname{Var}_{S \times \mathbb{G}_{m}}^{\mathbb{G}_{m}, n}\right)$ est défini dans [19, 2.5, 2.7] et [18, 2.2] comme le groupe abélien libre engendré par les classes d'isomorphismes des variétés $\left(X \rightarrow S \times \mathbb{G}_{m}, \sigma\right)$ de la catégorie $\operatorname{Var}_{S \times \mathbb{G}_{m}}^{\mathbb{G}_{m}, n}$, modulo les relations :

1. $\left[X \rightarrow S \times \mathbb{G}_{m}, \sigma\right]=\left[X^{\prime} \rightarrow S \times \mathbb{G}_{m}, \sigma\right]+\left[X \backslash X^{\prime} \rightarrow S \times \mathbb{G}_{m}, \sigma\right]$ pour $X^{\prime}$ un fermé de $X$ invariant sous $\mathbb{G}_{m}$,

2. $\left[X \times \mathbb{A}_{k}^{n} \rightarrow S \times \mathbb{G}_{m}, \sigma\right]=\left[X \times \mathbb{A}_{k}^{n} \rightarrow S \times \mathbb{G}_{m}, \sigma^{\prime}\right]$

lorsque $\sigma$ et $\sigma^{\prime}$ sont deux actions qui relèvent la même $\mathbb{G}_{m}$-action sur $X$ en actions affines sur le fibré

3. $\left[X \rightarrow S \times \mathbb{G}_{m}, \sigma\right]=\left[X \rightarrow S \times \mathbb{G}_{m}, \sigma_{l}\right]$ où $\sigma_{l}(\lambda, x)$ est égal à $\sigma\left(\lambda^{l}, x\right)$ pour tout $\lambda$ dans $\mathbb{G}_{m}$, pour tout $x$ dans $X$ et pour tout entier $l$ strictement positif.

Pour tout entier $n$ strictement positif, le produit fibré au dessus de $S \times \mathbb{G}_{m}$ muni de l'action diagonale, induit naturellement un produit dans la catégorie $\operatorname{Var}_{S \times \mathbb{G}_{m}}^{\mathbb{G}_{m}, n}$ et une structure d'anneau sur le groupe $K_{0}\left(\operatorname{Var}_{S \times \mathbb{G}_{m}}^{\mathbb{G}_{m}, n}\right)$. L'élément unité pour le produit, noté $1_{S \times \mathbb{G}_{m}}$, est la classe du morphisme identité sur $S \times \mathbb{G}_{m}$ où $\mathbb{G}_{m}$ agit trivialement sur $S$ et par translation sur lui même. En particulier, l'anneau $K_{0}\left(\operatorname{Var}_{S \times \mathbb{G}_{m}}^{\mathbb{G}_{m}, n}\right)$ a une structure de $K_{0}\left(\operatorname{Var}_{k}\right)$-module.

1.1.3. Localisation. - Pour tout entier $n$ strictement positif, dans l'anneau $K_{0}\left(\operatorname{Var}_{S \times \mathbb{G}_{m}}^{\mathbb{G}_{m}, n}\right)$ on note $\mathbb{L}$ la classe $\left[\mathbb{A}_{k}^{1} \times S \times \mathbb{G}_{m}, \pi_{S \times \mathbb{G}_{m}}, \tau_{n}\right]$ où $\pi_{S \times \mathbb{G}_{m}}$ est la projection sur $S \times \mathbb{G}_{m}$ et $\tau_{n}(\lambda,(a, x, \mu))$ est égal à $\left(a, x, \lambda^{n} \mu\right)$, pour tout $\lambda$ dans $\mathbb{G}_{m}$ et $(a, x, \mu)$ dans $\mathbb{A}_{k}^{1} \times S \times \mathbb{G}_{m}$. On note alors $\mathcal{M}_{S \times \mathbb{G}_{m}}^{\mathbb{G}_{m}, n}$ l'anneau localisé $K_{0}\left(\operatorname{Var}_{S \times \mathbb{G}_{m}}^{\mathbb{G}_{m}, n}\right)\left[\mathbb{L}^{-1}\right]$. 
1.1.4. Image directe, image inverse. - Soit $f: S \rightarrow S^{\prime}$ un morphisme de variétés. La composition par $f$ fournit le morphisme de groupes image directe $f_{!}: \mathcal{M}_{S \times \mathbb{G}_{m}}^{\mathbb{G}_{m}, n} \rightarrow \mathcal{M}_{S^{\prime} \times \mathbb{G}_{m}}^{\mathbb{G}_{m}, n}$. Le produit fibré au dessus de $S^{\prime}$ fournit le morphisme d'anneaux image inverse $f^{-1}: \mathcal{M}_{S^{\prime} \times \mathbb{G}_{m}}^{\mathbb{G}_{m}, n} \rightarrow \mathcal{M}_{S \times \mathbb{G}_{m}}^{\mathbb{G}_{m}, n}$. On peut définir ces morphismes au niveau du $K_{0}$.

1.1.5. Limite inductive. - Les catégories $\operatorname{Var}_{S \times \mathbb{G}_{m}}^{\mathbb{G}_{m}, n}$ et les anneaux $K_{0}\left(\operatorname{Var}_{S \times \mathbb{G}_{m}}^{\mathbb{G}_{m}, n}\right)$ et $\mathcal{M}_{S \times \mathbb{G}_{m}}^{\mathbb{G}_{m}, n}$ forment un système inductif induit par l'ordre partiel donné par la divisibilité entre les entiers. On note alors $\operatorname{Var}_{S \times \mathbb{G}_{m}}^{\mathbb{G}_{m}}, K_{0}\left(\operatorname{Var}_{S \times \mathbb{G}_{m}}^{\mathbb{G}_{m}}\right)$ et $\mathcal{M}_{S \times \mathbb{G}_{m}}^{\mathbb{G}_{m}}$ les colimites. On remarquera en particulier que toutes les classes $\left[\mathbb{A}_{k}^{1} \times S \times \mathbb{G}_{m}, \pi_{S \times \mathbb{G}_{m}}, \tau_{n}\right]$ sont identifiées en un élément noté $\mathbb{L}$ par la suite.

1.1.6. Séries rationnelles. - Soit $A$ l'un des anneaux $\mathbb{Z}\left[\mathbb{L}, \mathbb{L}^{-1}\right], \mathcal{M}_{S \times \mathbb{G}_{m}}^{\mathbb{G}_{m}}$ et $\mathbb{Z}\left[\mathbb{L}, \mathbb{L}^{-1},\left(1 /\left(1-\mathbb{L}^{-i}\right)\right)_{i>0}\right]$. On note $A[[T]]_{s r}$ le sous $A$-module de $A[[T]]$ engendré par 1 et par un produit fini de termes $\mathbb{L}^{e} T^{i} /\left(1-\mathbb{L}^{e} T^{i}\right)$ notés $p_{e, i}(T)$ avec $e$ dans $\mathbb{Z}$ et $i$ dans $\mathbb{N}_{>0}$. Il existe un unique morphisme $A$-linéaire

$$
\lim _{T \rightarrow \infty}: A[[T]]_{s r} \rightarrow A
$$

tel que pour toute famille $\left(e_{i}, j_{i}\right)_{i \in I}$ de $\mathbb{Z} \times \mathbb{N}_{>0}$ avec $I$ fini ou vide, $\lim _{T \rightarrow \infty}\left(\prod_{i \in I} p_{e_{i}, j_{i}}(T)\right)$ vaut $(-1)^{|I|}$.

1.1.7. Cône convexe polyédral. - Soit $I$ un ensemble fini. Un cône convexe polyédral rationnel de $\mathbb{R}^{*|I|}$ est une partie convexe de $\mathbb{R}^{*|I|}$ définie par un nombre fini d'inégalités linéaires à coefficients entiers du type $a \leq 0$ et $b>0$ et stable sous la multiplication de $\mathbb{R}_{>0}$.

1.1.8. Lemme de rationnalité. - Le lemme suivant est bien connu, on peut se référer à [17, 2.1.5] et [19, 2.9].

Lemme 1.2. - Soit I un ensemble fini. Soit $\Delta$ un cône polyédral rationnel convexe dans $\mathbb{R}_{>0}^{I}$. On note $\bar{\Delta}$ l'adhérence de $\Delta$ dans $\mathbb{R}_{\geq 0}^{I}$. Soit l et $\nu$ deux formes linéaires sur $\mathbb{Z}^{I}$, supposons $l$ strictement positive et $\nu$ positive sur $\bar{\Delta} \backslash\{0\}$. Dans l'anneau $\mathbb{Z}\left[\mathbb{L}, \mathbb{L}^{-1}\right][[T]]$ notons $S_{\Delta, l, \nu}(T)$ les séries $\sum_{k \in \Delta \cap \mathbb{N}_{>0}^{I}} T^{l(k)} \mathbb{L}^{-\nu(k)}$. Lorsque $\Delta$ est ouvert dans son propre espace vectoriel engendré et $\bar{\Delta}$ est engendré par une famille de vecteurs que l'on peut compléter en une $\mathbb{Z}$-base $d u \mathbb{Z}$-module $\mathbb{Z}^{I}$, les séries $S_{\Delta, l, \nu}(T)$ sont rationnelles et leur limite $\lim _{T \rightarrow \infty} S_{\Delta, l, \nu}(T)$ est égale à $(-1)^{\operatorname{dim}(\Delta)}$. Dans le cas général, par additivité par rapport à l'union disjointe des cônes, grâce à l'hypothèse de positivité, les séries $S_{\Delta, l, \nu}(T)$ sont rationnelles et leur limite est égale à la caractéristique d'Euler à support compact du cône $\Delta$. 
Notons que dans $[19,2.9]$, les formes linéaires $l$ et $\nu$ sont supposées strictement positives sur $\bar{\Delta} \backslash\{0\}$, mais la même preuve permet de supposer seulement $l$ strictement positive sur $\bar{\Delta} \backslash\{0\}$.

\subsection{Arcs}

1.2.1. Espaces d'arcs. - Soit $X$ une $k$-variété. On note $L_{n}(X)$ l'espace des $n$-jets. Cet objet est un $k$-schéma dont les $K$-points rationnels, sont les morphismes Spec $K[t] / t^{n+1} \rightarrow X$, pour tout corps $K$ contenant $k$. Il existe des morphismes canoniques $L_{n+1}(X) \rightarrow L_{n}(X)$. Ces morphismes sont des fibrés en $\mathbb{A}_{k}^{d}$ quand $X$ est lisse de dimension pure $d$. L' espace des arcs noté $L(X)$ est la limite projective de ce système. On note $\pi_{n}: L(X) \rightarrow L_{n}(X)$ les morphismes canoniques. On pourra par exemple se référer à [11] et [24].

1.2.2. Action et coefficient angulaire. - Le groupe $\mathbb{G}_{m}$ agit canoniquement sur $L_{n}(X)$ et sur $L(X)$ par $\lambda . \varphi(t)$ égal à $\varphi(\lambda t)$. Dans toute la suite de l'article on note $\sigma$ cette action.

Pour un élément $\varphi$ de $K[[t]]$ ou de $K[t] / t^{n+1}$, on désigne par $\operatorname{ord}_{t}(\varphi)$ la valuation de $\varphi$ et $\operatorname{par} \operatorname{ac}(\varphi)$ son premier coefficient non nul. Par convention $\operatorname{ac}(0)$ est nul. Le scalaire $\operatorname{ac}(\varphi)$ est appelé coefficient angulaire de $\varphi$.

1.2.3. Ordre de contact. - Soit $X$ une variété et $F$ un sous-schéma fermé de $X$. On note $I_{F}$ l'idéal des fonctions s'annulant sur $F$. On désigne par $\operatorname{ord}_{t} F$ la fonction qui a tout arc $\varphi$ dans $L(X)$ associe la borne inférieure $\inf \operatorname{ord}_{t} g(\varphi)$ où $g$ parcourt les sections locales de $I_{F}$ en l'origine de $\varphi$.

1.3. Le morphisme fibre de Milnor motivique. - Considérons $X$ une variété et un morphisme $f: X \rightarrow \mathbb{A}_{k}^{1}$. Grâce au théorème de factorisation faible, Bittner [1] étend la fibre de Milnor motivique en un morphisme défini sur tout l'anneau de Grothendieck $M_{X}$. Guibert, Loeser et Merle [19] donnent une construction différente basée sur l'intégration motivique. Nous l'expliquons ci-dessous et nous l'utiliserons par la suite.

DÉfinition 1.3. - Pour une variété $X$ lisse de dimension $d, U$ un ouvert partout dense de $X, F$ le complémentaire de $U$ dans $X$ et $f: X \rightarrow \mathbb{A}_{k}^{1}$ un morphisme, on considère pour deux entiers $n$ et $\gamma$ strictement positifs, l'espace d'arcs

$$
\mathscr{X}_{n}^{\gamma}(f):=\left\{\varphi \in L(X) \mid \operatorname{ord}_{t} f(\varphi)=n, \operatorname{ord}_{t} F \cdot \varphi \leq n \gamma\right\}
$$

muni de la flèche « origine, coefficient angulaire» notée $\left(\pi_{0}, \operatorname{ac}(f)\right)$ vers le produit $X_{0}(f) \times \mathbb{G}_{m}$ et de l'action standard de $\mathbb{G}_{m}$ sur les arcs. On considère ensuite la fonction zêta modifiée

$$
Z_{f, U}^{\gamma}(T):=\sum_{n \geq 1} \mu\left(\mathscr{X}_{n}^{\gamma}(f)\right) T^{n} \in \mathcal{M}_{X_{0}(f) \times \mathbb{G}_{m}}^{\mathbb{G}_{m}}[[T]] .
$$



$3.8]$

On dispose du théorème de rationalité à la Denef-Loeser démontré en [19,

Proposition 1.4. - Soit $U$ un ouvert dense d'une variété $X$ lisse et de dimension pure d. Soit $f: X \rightarrow \mathbb{A}_{k}^{1}$ un morphisme. Il existe un entier $\gamma_{0}$ tel que pour tout entier $\gamma$ supérieur à $\gamma_{0}$, les séries $Z_{f, U}^{\gamma}(T)$ sont rationnelles et leur limite est indépendante de $\gamma$. On désignera par $S_{f, U}$ la limite $-\lim _{T \rightarrow \infty} Z_{f, U}^{\gamma}(T)$. De plus si $X_{0}(f)$ est nulle part dense dans $X$, et $h: Y \rightarrow X$ est une logrésolution de $\left(X, F \cup X_{0}(f)\right)$ on dispose alors d'une formule d'image directe

$$
S_{f, U}=h_{!}\left(S_{f \circ h, h^{-1}(U)}\right) \in \mathcal{M}_{X_{0}(f) \times \mathbb{G}_{m}}^{\mathbb{G}_{m}} .
$$

Bittner, puis Guibert, Loeser et Merle prouvent alors le théorème d'extension à tout le groupe de Grothendieck ([1], [19, 3.9]) :

THÉORÈme 1.5. - Soit $X$ une variété et $f: X \rightarrow \mathbb{A}_{k}^{1}$ un morphisme. Il existe un unique morphisme de $\mathcal{M}_{k}$-modules $S_{f}: \mathcal{M}_{X} \rightarrow \mathcal{M}_{X_{0}(f) \times \mathbb{G}_{m}}^{\mathbb{G}_{m}}$ tel que pour tout morphisme propre $p: Z \rightarrow X$ avec $Z$ lisse, et pour toute partie $U$ ouverte et dense dans $Z, S_{f}([p: U \rightarrow X])$ vaut $p_{!}\left(S_{f \circ p, U}\right)$.

Remarque 1.6. - Soit $X$ une variété, $U$ un ouvert partout dense, $F$ son complémentaire et $f: X \rightarrow \mathbb{A}_{k}^{1}$ un morphisme. Si $f$ est lisse sur $X$ alors $S_{f}(X)$ est égal à la classe $\left[X_{0}(f) \times \mathbb{G}_{m}, i d, \tau\right]$ où $\tau$ est la translation. Si $F$ un fermé lisse de $X$, l'injection $i: F \rightarrow X$ est propre donc l'image $S_{f}([i: F \rightarrow X])$ est égale à $i_{!} S_{f \circ i}(F)$ c'est à dire à $i_{!} S_{f_{\mid F}}$. Par additivité, le résultat est vrai pour tout fermé de $X$. Par conséquent, si $f$ est lisse sur $X$ et en restriction au fermé $F$ alors $S_{f}(U)$ vaut $\left[U \cap X_{0}(f) \times \mathbb{G}_{m}, i d, \tau\right]$ où $\tau$ est la translation.

DÉfinition 1.7. - Considérons $X$ une variété de dimension $d$ et $f: X \rightarrow \mathbb{A}_{k}^{1}$ un morphisme. Pour $U$ un ouvert de $X$ et $F$ le fermé complémentaire on appelle cycles proches motiviques l'objet $S_{f}(U)$ et cycles évanescents motiviques l'objet

$$
S_{f}^{\Phi}(U):=(-1)^{d-1}\left(S_{f}(U)-\left[U \cap X_{0}(f) \times \mathbb{G}_{m}, i d, \tau\right]\right) .
$$

Ce sont des éléments de l'anneau $\mathcal{M}_{X_{0}(f) \times \mathbb{G}_{m}}^{\mathbb{G}_{m}}$. Par la remarque précédente, si $f$ est lisse sur $X$ et en restriction au fermé $F$ alors les cycles évanescents motiviques $S_{f}^{\Phi}(U)$ sont nuls.

1.4. Réalisations. - On se référera à [11], [19, 3.16, 6.1] et [32]. On suppose ici $k$ égal à $\mathbb{C}$. 
1.4.1. Réalisation de Hodge. - On notera $S H$ la catégorie abélienne des structures de Hodge et $K_{0}(S H)$ son anneau de Grothendieck correspondant. Rappelons qu'une structure de Hodge mixte $V$ de filtration par le poids $W \bullet$ admet dans l'anneau $K_{0}(S H)$ une classe canonique [V] égale à $\sum_{m}\left[G r_{m}^{W}(V)\right]$ où pour tout $m,\left[G r_{m}^{W}(V)\right]$ est la classe de la structure de Hodge du gradué $G r_{m}^{W}(V)$ égal à $W_{m}(V) / W_{m-1}(V)$. Il existe un morphisme canonique

$$
\chi_{h}: M_{\mathbb{C}} \rightarrow K_{0}(S H)
$$

qui associe à la classe d'une variété $X$, l'élément $\sum_{i}(-1)^{i}\left[H_{c}^{i}(X, \mathbb{Q})\right]$ dans $K_{0}(S H)$ où pour tout $i$, la classe $\left[H_{c}^{i}(X, \mathbb{Q})\right]$ est la classe de la structure de Hodge mixte de Deligne sur $H_{c}^{i}(X, \mathbb{Q})$.

On notera $S H^{\text {mon }}$ la catégorie abélienne des structures de Hodge munies d'un endomorphisme quasi-unipotent et $K_{0}\left(S H^{\mathrm{mon}}\right)$ son anneau de Grothendieck. Il existe un morphisme canonique

$$
\chi_{h}: \mathcal{M}_{\mathbb{G}_{m}}^{\mathbb{G}_{m}} \rightarrow K_{0}\left(S H^{\mathrm{mon}}\right)
$$

défini comme suit. Si $[X]$ est la classe de $f: X \rightarrow \mathbb{G}_{m}$ dans $\mathcal{M}_{\mathbb{G}_{m}}^{\mathbb{G}_{m}}$ avec $X$ connexe, et $f$ homogène par rapport à l'action $\sigma$ de $\mathbb{G}_{m}$, alors $f$ est une fibration localement triviale pour la topologie complexe. De plus si le poids est $n$ alors pour tout $x$ dans $X$ l'application

$$
\begin{array}{ccc}
{[0,1]} & \rightarrow & X \\
t & \mapsto \sigma(\exp (2 i \pi t / n), x)
\end{array}
$$

est une monodromie géométrique d'ordre fini autour de l'origine. La fibre de $f$ en 1 que l'on note $X_{1}$ est munie d'un automorphisme d'ordre fini $T_{f}$, et on pose

$$
\chi_{h}\left(\left[f: X \rightarrow \mathbb{G}_{m}, \sigma\right]\right):=\left(\sum_{i}(-1)^{i}\left[H_{c}^{i}\left(X_{1}, \mathbb{Q}\right), T_{f}\right]\right)
$$

Il existe une application linéaire appelée spectre de Hodge

$$
\begin{aligned}
\operatorname{sph}: K_{0}\left(S H^{\text {mon }}\right) & \rightarrow \mathbb{Z}[\mathbb{Q}] \quad:=\cup_{n \geq 1} \mathbb{Z}\left[t^{1 / n}, t^{-1 / n}\right] \\
{[H] } & \mapsto \operatorname{sph}([H]):=\sum_{\alpha \in \mathbb{Q} \cap[0,1[} t^{\alpha}\left(\sum_{p, q \in \mathbb{Z}} \operatorname{dim} H_{\alpha}^{(p, q)} t^{p}\right)
\end{aligned}
$$

où $H_{\alpha}^{p, q}$ est l'espace caractéristique de $H^{p, q}$ par rapport à la valeur propre $e^{2 i \pi \alpha}$, pour une structure de Hodge munie d'un endomorphisme quasi-unipotent $H$. On note alors Sp la composée sph $\circ \chi_{h}$. 
1.4.2. Réalisation au niveau des modules de Hodge mixtes. — On peut se référer au chapitre 14 de [32]. Pour toute variété algébrique complexe $X$, on note $M H M_{X}$ la catégorie abélienne des modules de Hodge mixtes sur $X$ et l'on note $K_{0}\left(M H M_{X}\right)$ l'anneau de Grothendieck correspondant. Nous considérerons aussi $M H M_{X}^{\text {mon }}$ la catégorie abélienne des modules de Hodge mixtes sur $X$ munis d'un automorphisme d'ordre fini et $K_{0}\left(M H M_{X}^{\text {mon }}\right)$ son anneau de Grothendieck correspondant.

1.4.2.1. Lien avec les faisceaux pervers. - Par définition des modules de Hodge mixtes, il existe un foncteur fidèle $\operatorname{rat}_{X}: D^{b}(M H M(X)) \rightarrow D_{c}^{b}(X, \mathbb{Q})$ tel que la catégorie $M H M(X)$ corresponde à la catégorie $\operatorname{Perv}(X, \mathbb{Q})$. Pour tout module de Hodge mixte $M$ sur $X$, on appelle $\operatorname{rat}_{X} M$ son faisceau pervers rationnel sous jacent. On pourra consulter l'axiome A [32, 14.1].

1.4.2.2. Lien avec les structures de Hodge mixtes. - Par construction des modules de Hodge mixtes, il existe une équivalence de catégories entre la catégorie $M H M_{\mathrm{SpecC}}^{\mathrm{mon}}$ des modules de Hodge mixtes supportés par le point et munis d'un endomorphisme quasi-unipotent et la catégorie $S H M^{\text {mon }}$ des structures de Hodge mixtes munies d'un endomorphisme quasi-unipotent. On notera alors $\Phi: K_{0}\left(M H M_{\text {Spec }}^{\text {mon }}\right) \rightarrow K_{0}\left(S H^{\text {mon }}\right)$ le morphisme d'anneaux induit par l'application canonique qui a une structure de Hodge mixte munie d'un endomorphisme quasi-unipotent associe sa classe dans l'anneau $K_{0}\left(S H^{\mathrm{mon}}\right)$. On pourra consulter l'axiome B de [32, 14.1, 14.61].

1.4.2.3. Réalisation au niveau des modules de Hodge mixtes. - Il existe un morphisme de réalisation entre les variétés et les modules de Hodge mixtes

$$
H: K_{0}\left(\operatorname{Var}_{X}\right) \rightarrow K_{0}\left(M H M_{X}\right)
$$

En effet, par additivité, il existe un unique morphisme d'anneaux $H$ tel que pour tout $p: Z \rightarrow X$ avec $Z$ lisse, $H([p: Z \rightarrow X])$ est la classe de l'image directe à support compact $R p_{!}\left(\mathbf{Q}_{Z}\right)$ dans $K_{0}\left(M H M_{X}\right)$ avec $\mathbf{Q}_{Z}$ le module de Hodge trivial sur $Z$. L'image de la classe $\mathbb{L}$ est égale à la classe $\left[\mathbf{Q}_{X}(-1)\right]$. Par conséquent ce morphisme s'étend en un morphisme $\mathcal{M}_{k}$-linéaire

$$
H: \mathcal{M}_{X} \rightarrow K_{0}\left(M H M_{X}\right)
$$

avec $K_{0}\left(M H M_{X}\right)$ vu comme un $\mathcal{M}_{k}$-module via sa structure de $K_{0}\left(M H M_{\text {Spec }}\right)$-module, et la réalisation de Hodge $H: \mathcal{M}_{k} \rightarrow K_{0}\left(M H M_{\text {Spec }}\right)$. Il existe de même un morphisme naturel compatible dans le cas équivariant (voir [19, 2.6, 3.16])

$$
H: \mathcal{M}_{X \times \mathbb{G}_{m}}^{\mathbb{G}_{m}} \rightarrow K_{0}\left(M H M_{X}^{\text {mon }}\right) .
$$

Notons que la composée des morphismes $\Phi$ et $H$ redonne les morphismes d'anneaux $\chi_{h}$. 
1.4.2.4. Cycles proches. - Soit $f: X \rightarrow \mathbb{A}_{\mathbb{C}}^{1}$ une fonction. Dans [38] M.Saito construit un foncteur cycles proches $\Psi_{f}$ et un foncteur cycles évanescents $\Phi_{f}$ de la catégorie $M H M_{X}$ vers la catégorie $M H M_{X_{0}(f)}^{\operatorname{mon}}$ tel que les faisceaux pervers sous jacents $\operatorname{rat}_{X} \Psi_{f}\left(\mathbf{Q}_{X}\right)$ et $\operatorname{rat}_{X} \Phi_{f}\left(\mathbf{Q}_{X}\right)$ soient égaux à $\psi_{f}\left(\mathbb{Q}_{X}\right)[-1]$ et $\phi_{f}\left(\mathbb{Q}_{X}\right)[-1]$. On dispose alors du morphisme cycles proches

$$
\Psi_{f}: K_{0}\left(M H M_{X}\right) \rightarrow K_{0}\left(M H M_{X_{0}(f)}^{\operatorname{mon}}\right) .
$$

1.4.2.5. Compatibilité entre la fibre de Milnor motivique et le foncteur cycles proches. — Denef et Loeser [9, 4.2.1], puis Guibert, Loeser et Merle [19, 3.17] montrent le théorème de compatibilité entre la fibre de Milnor motivique et le morphisme cycles proches, modulo la réalisation de Hodge :

THÉORÈME 1.8. - Le diagramme commutatif suivant est commutatif :

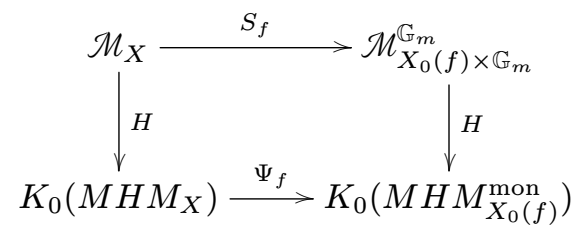

Par composition avec le morphisme rat $_{X}$, on obtient un morphisme $\mathcal{M}_{X} \rightarrow$ $K_{0}\left(\operatorname{Perv}_{X_{0}}^{\text {mon }}\right)$ tel que $S_{f}(X)$ et $S_{f}^{\phi}(X)$ se réalisent en la classe $\left[\psi_{f}\left(\mathbb{Q}_{X}\right)[-1]\right]$ et $\left[\phi_{f}\left(\mathbb{Q}_{X}\right)[-1]\right]$ et pour tout ouvert $U$ lisse de $X, S_{f}(U)$ et $S_{f}^{\phi}(U)$ se réalisent en la classe $\left[\psi_{f}\left(R i_{!} \mathbb{Q}_{U}\right)[-1]\right]$ et $\left[\phi_{f}\left(R i_{!} \mathbb{Q}_{U}\right)[-1]\right]$ où $i$ est l'injection de $U$ dans $X$.

\section{Fibre de Milnor motivique à l'infini}

Soit $k$ un corps de caractéristique 0 , soit $U$ une variété algébrique lisse sur $k$ et $f: U \rightarrow \mathbb{A}_{k}^{1}$ une application régulière.

On considère $j: \mathbb{A}_{k}^{1} \rightarrow \mathbb{P}_{k}^{1}$ l'injection qui a un élément $a$ associe $[1: a]$. On désignera par $\infty$ l'élément $[0: 1]$ et on notera par abus $a$ l'élément $[1: a]$.

2.1. Compactification. - On appelle compactification de $f$ la donnée d'une variété $X$, d'une immersion ouverte dominante $i$ et d'une application propre $\hat{f}$ telles que le diagramme suivant commute

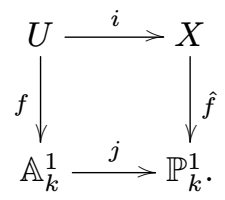

L'existence d'une compactification est assurée par un théorème de Nagata, on peut par exemple se référer à [7]. 
2.1.1. Notations. - Pour une compactification $(X, i, \hat{f})$ on note $U_{X}$ l'ouvert $i(U), U_{X}^{*}$ l'ouvert $i(U) \backslash i\left(f^{-1}(0)\right), F_{X}$ le fermé à l'infini $X \backslash U_{X}$ et $X_{a}$ la fibre $\hat{f}^{-1}(a)$ pour tout $a$ dans $\mathbb{P}_{k}^{1}$. On notera enfin par abus $1 / \hat{f}$ l'application régulière qui prolonge $1 / f$ définie sur $X \backslash X_{0}$ et à valeurs dans $\mathbb{A}_{k}^{1}$.

2.1.2. Compactifications lisses. - Soit $(X, i, \hat{f})$ une compactification. L'ouvert $U$ est lisse et $i$ est une immersion ouverte, le lieu singulier $X_{\text {Sing }}$ est donc contenu dans le fermé à l'infini $F_{X}$. De plus, par le théorème de résolution des singularités d'Hironaka [20], on peut déduire de $(X, i, \hat{f})$ une compactification $\left(Z, i_{Z}, f_{Z}\right)$ où $Z$ est lisse sur $k$ et est birationnellement équivalente à $X$. On considère pour cela $(Z, E, h)$ une log-résolution de $\left(X, X_{\text {Sing }}\right)$. La variété $Z$ est lisse, l'ouvert $i(U)$ est inclus dans $X \backslash X_{\text {Sing }}$, donc $h$ est inversible sur $i(U)$, le morphisme $h^{-1} \circ i$ est une immersion ouverte, comme composée d'immersions ouvertes, l'ouvert $i(U)$ est dense et $h$ est un isomorphisme de $Z \backslash E$ dans $X \backslash X_{\text {Sing }}$, donc $h^{-1}(i(U))$ est un ouvert dense dans $Z \backslash E$ donc dans $Z$. Le morphisme $\hat{f} \circ h$ est propre comme composée d'applications propres. Enfin la composée $(\hat{f} \circ h) \circ\left(h^{-1} \circ i\right)$ est égale à $\hat{f} \circ i$ elle même égale à $j \circ f$.

2.2. Fibre de Milnor à l'infini. - On suppose, dans cette sous section, $k$ égal à $\mathbb{C}$.

2.2.1. Fibre de Milnor à l'infini et ensemble de bifurcation. — Le théorème suivant est un corollaire bien connu de l'existence de stratifications de Whitney et du théorème de fibration de Thom-Mather [33], [43] et [44].

ThÉORÈme 2.1. - Pour toute variété algébrique complexe lisse $U$, pour toute application régulière $f: U \rightarrow \mathbb{A}_{\mathbb{C}}^{1}$, il existe un ensemble fini minimal $\mathcal{B}$, appelé ensemble de bifurcation tel que $f: U \backslash f^{-1}(\mathcal{B}) \rightarrow \mathbb{A}_{\mathbb{C}}^{1} \backslash \mathcal{B}$ soit une fibration topologique localement triviale. En particulier, il existe $R>0$ tel que $f$ de $U \backslash f^{-1}(\bar{D}(0, R))$ vers $\mathbb{A}_{\mathbb{C}}^{1} \backslash \bar{D}(0, R)$ est une fibration topologique localement triviale. On appelle fibre de Milnor à l'infini la fibre générale de cette dernière fibration.

L'ensemble de bifurcation contient les valeurs critiques du morphisme $f$. Néanmoins l'inclusion peut-être stricte. On peut considérer l'exemple de Broughton [4], $x(x y-1)$ : cette fonction est lisse sur $\mathbb{C}^{2}$ et toutes les fibres sont lisses et connexes exceptée la fibre en 0 . On appelle valeur atypique toute valeur de bifurcation non contenue dans le discriminant. Nous définirons dans la partie 4 des valeurs motiviquement atypiques et un ensemble de bifurcation motivique. 
2.2.2. Structure de Hodge mixte limite. - Pour tout $t$, les espaces de cohomologie à support compact $H_{c}^{*}\left(f^{-1}(t), \mathbb{Q}\right)$ de la fibre en $t$ sont munis d'une structure de Hodge mixte naturelle [8]. Ces structures de Hodge mixtes sont les fibres d'une variation de structures de Hodge mixtes sur le complémentaire de l'union $\mathscr{B} \cup\{\infty\}$ dans $\mathbb{P}_{\mathbb{C}}^{1}$. Steenbrink et Zucker [40] puis M. Saito [38] ont montré comment construire une structure de Hodge mixte limite lorsque $t$ tend vers l'infini ou vers une valeur de bifurcation. Sabbah [35] l'a retrouvée en considérant la transformation de Fourier sur des modules convenables sur l'anneau des opérateurs différentiels. Une bonne référence pour cela est [32].

En particulier cette structure de Hodge mixte peut être obtenue de la manière suivante. Soit $(X, i, \hat{f})$ une compactification lisse et $\Psi_{1 / \hat{f}}\left(R i_{!} \mathbf{Q}_{U}\right)$ le module de Hodge mixte sur le faisceau des cycles proches en $t=\infty$ et évalué sur le module $R i_{!} \mathbf{Q}_{U}$. La structure de Hodge mixte limite sur $H_{c}^{k}\left(f^{-1}(t), \mathbb{Q}\right)$ s'identifie à la structure de Hodge mixte de $\mathbb{H}_{c}^{k}\left(\hat{f}^{-1}(\infty), \psi_{1 / \hat{f}}\left(R i_{!} \mathbb{Q}_{U}\right)\right)[35,5.5]$. On obtient ce groupe d'hypercohomologie en prenant le faisceau pervers sous jacent du module de Hodge mixte $R p_{!}\left(\Psi_{1 / \hat{f}}\left(R i_{!} \mathbf{Q}_{U}\right)\right)$ où $R p_{\text {! }}$ est l'image directe sur le point (voir 1.4.2.1).

Le spectre de cette structure limite est un invariant de $f$ appelé spectre à l'infini (voir le paragraphe 1.4.1). Il a notamment été étudié (souvent pour la cohomologie sans support) par Brélivet [2] et [3], Dimca [12], Garcia-Lopez et Némethi [14], [16] et [15], Némethi et Sabbah [27] et Sabbah [35] et [37].

REMARQUE 2.2. - La monodromie naturelle du foncteur des cycles proches $\psi_{1 / \hat{f}}$ est l'inverse de la monodromie le long d'un grand cercle orienté positivement dans $\mathbb{A}_{\mathbb{C}}^{1} \operatorname{voir}(1.9)[35]$.

2.3. Fibre de Milnor motivique à l'infini. - Considérons $(X, i, \hat{f})$ une compactification de $f$. Pour tout couple d'entiers strictement positifs $n$ et $\gamma$, considérons l'espace d'arcs

$$
\mathscr{X}_{n}^{\gamma}(\hat{f})=\left\{\begin{array}{l|l}
\varphi \in L(X) \backslash L(F) & \begin{array}{l}
\operatorname{ord}_{t} F . \varphi \leq n \gamma \\
\operatorname{ord}_{t} 1 / \hat{f}(\varphi(t))=n
\end{array}
\end{array}\right\} .
$$

Cet espace d'arcs est mesurable, même si la compactification est singulière. L'ordre de contact des arcs avec le lieu singulier étant borné, sa mesure appartient à l'anneau de Grothendieck $\mathcal{M}_{X_{\infty} \times \mathbb{G}_{m}}^{\mathbb{G}_{m}}$, sans nécessité de complétion [10, Lemme 4.1].

On considère alors la fonction zêta motivique modifiée :

$$
Z_{1 / \hat{f}, U_{X}^{*}}^{\gamma}(T)=\sum_{n \geq 1} \mu\left(\mathscr{X}_{n}^{\gamma}(\hat{f})\right) T^{n} \in \mathcal{M}_{X_{\infty} \times \mathbb{G}_{m}}^{\mathbb{G}_{m}}[[T]] .
$$


Proposition 2.3. - Pour toute compactification $(X, i, \hat{f})$ de $f$, il existe un entier $\gamma_{0}$, tel que pour tout entier $\gamma$ supérieur à $\gamma_{0}$, la fonction zêta modifée $Z_{1 / \hat{f}, U_{X}^{*}}^{\gamma}(T)$ est rationnelle et sa limite ne dépend pas de $\gamma$. L'opposé de cette limite est $S_{1 / \hat{f}}\left(U_{X}^{*}\right)$ qui appartient à l'anneau $\mathcal{M}_{X_{\infty} \times \mathbb{G}_{m}}^{\mathbb{G}_{m}}$ (cf théorème 1.5). De plus si $(Z, E)$ est une log-résolution du couple $\left(X, F_{X}\right)$ alors

$$
S_{1 / \hat{f}}(i: U \rightarrow X)=h_{!} S_{1 / \hat{f} \circ h}\left(h^{-1}\left(U_{X}^{*}\right)\right) .
$$

Démonstration. - Si la compactification $(X, i, \hat{f})$ est singulière, son lieu singulier est alors contenu dans le fermé à l'infini $F_{X}$. La fibre de la valeur infini est elle même contenue dans $F_{X}$. La preuve de la proposition 1.4 (voir $[19,3.8]$ ) montre qu'il existe un entier $\gamma_{0}$, tel que pour tout entier $\gamma$ supérieur à $\gamma_{0}$, la fonction zêta modifée $Z_{1 / \hat{f}, U_{X}^{*}}^{\gamma}(T)$ est rationnelle et sa limite ne dépend pas de $\gamma$ : En effet, elle utilise une log-résolution $(Z, E)$ du couple $\left(X, F_{X}\right)$ et repose essentiellement sur la formule de changement de variables de Denef et Loeser [10] valable pour $Y$ lisse et qui garde un sens pour $X$ singulier. En particulier la série $Z_{1 / \hat{f}, U_{X}^{*}}^{\gamma}(T)$ a une limite quand $T$ tend vers l'infini, dont l'opposé est égal à $h_{!} S_{1 / \hat{f} \circ h}\left(h^{-1}\left(U_{X}^{*}\right)\right)$, lui-même égal à $S_{1 / \hat{f}}\left(i_{X}: U \rightarrow X\right)$ qui appartient à l'anneau $\mathcal{M}_{X_{\infty} \times \mathbb{G}_{m}}^{\mathbb{G}_{m}}$

En prenant l'image directe sur le point on obtient le résultat d'indépendance suivant

THÉORÈme 2.4. - Soient $\left(X, i_{X}, f_{X}\right)$ et $\left(Y, i_{Y}, f_{Y}\right)$ deux compactifications. Après image directe, on dispose de l'égalité

$$
f_{Y !} S_{1 / f_{Y}}\left(U_{Y}^{*}\right)=f_{X !} S_{1 / f_{X}}\left(U_{X}^{*}\right) \in \mathcal{M}_{\{\infty\} \times \mathbb{G}_{m}}^{\mathbb{G}_{m}}
$$

Cette valeur est appelée fibre de Milnor motivique à l'infini du morphisme $f$, elle est notée $S_{f, \infty}$ et est indépendante de toute compactification.

REMARQUe 2.5. - La fibre de Milnor motivique à l'infini est construite dans une compactification fixée à partir d'arcs dont l'origine appartient à la fibre à l'infini et est indépendante de la compactification. Cet objet ne dépend donc pas de l'ouvert $U$ initial, mais seulement de la restriction de $f$ au dessus d'un voisinage de l'infini. En particulier lorsque l'on retire à $U$ les fibres des valeurs de bifurcation, le morphisme $f: U \backslash f^{-1}(B) \rightarrow \mathbb{A}_{k}^{1}$ est lisse mais sa fibre de Milnor motivique à l'infini est celle de $f: U \rightarrow \mathbb{A}_{k}^{1}$.

REMARQUe 2.6. - Par la suite s'il n'y a aucun risque de confusion on notera $\mathcal{M}_{\mathbb{G}_{m}}^{\mathbb{G}_{m}}$ l'anneau $\mathcal{M}_{\{\infty\} \times \mathbb{G}_{m}}^{\mathbb{G}_{m}}$. 
REmARque 2.7. - Lorsque $k$ est le corps des nombres complexes, si l'on souhaite considérer la monodromie à l'infini le long d'un grand cercle orienté positivement dans $\mathbb{A}_{\mathbb{C}}^{1}$, il faut appliquer à $S_{f, \infty}$ l'opérateur inv de $\mathcal{M}_{\mathbb{G}_{m}}^{\mathbb{G}_{m}}$ sur lui même qui a une classe $\left[A \stackrel{l}{\rightarrow} \mathbb{G}_{m}, \sigma\right]$ associe la classe $\left[A \stackrel{\text { invol }}{\rightarrow} \mathbb{G}_{m}, \sigma\right]$ avec pour tout $\lambda$ dans $\mathbb{G}_{m}, \operatorname{inv}(\lambda)$ est égal à $\lambda^{-1}(\operatorname{cf} 2.2)$.

Démonstration. - La preuve de ce théorème d'indépendance repose sur un corollaire bien connu du théorème d'Hironaka :

ThÉORÈme 2.8. - Soit $X$ et $Y$ deux variétés, munies d'un morphisme propre $f_{X}: X \rightarrow \mathbb{P}_{k}^{1}$ et $f_{Y}: Y \rightarrow \mathbb{P}_{k}^{1}$. Soit $F_{X}$ un fermé de $X$ et $F_{Y}$ un fermé de $Y$, tels qu'il existe un isomorphisme $\Psi$ de $X \backslash F_{X}$ vers $Y \backslash F_{Y}$ vérifiant l'égalité $f_{X}=f_{Y} \circ \Psi$.

Il existe un quadruplet $\left(Z, E, h_{X}, h_{Y}\right)$ tel que le triplet $\left(Z, E, h_{X}\right)$ est une logrésolution du couple $\left(X, F_{X}\right)$, le triplet $\left(Z, E, h_{Y}\right)$ est une log-résolution du couple $\left(Y, F_{Y}\right)$ et la composée $f_{X} \circ h_{X}$ est égale à la composée $f_{Y} \circ h_{Y}$.

Démonstration. - On note $\Gamma$ le graphe de $\Psi$. Comme le morphisme $f_{X}$ est égal à la composée $f_{Y} \circ \Psi$, ce graphe est contenu dans le produit fibré $\left(X \times_{\mathbb{P}_{k}^{1}}\right.$ $\left.Y, p_{X}, p_{Y}\right)$. On considère $\bar{\Gamma}$, son adhérence dans $X \times_{\mathbb{P}_{k}^{1}} Y$. Le graphe $\Gamma$ est un ouvert lisse de $\bar{\Gamma}$ pour la topologie induite. En effet $\Gamma$ est égal à $p_{X}^{-1}\left(X \backslash F_{X}\right) \cap$ $p_{Y}^{-1}\left(Y \backslash F_{Y}\right) \cap \bar{\Gamma}$ et par continuité des projections, $p_{X}^{-1}\left(X \backslash F_{X}\right)$ et $p_{Y}^{-1}\left(Y \backslash F_{Y}\right)$ sont ouverts. Par le théorème de résolution des singularités d'Hironaka, considérons $(Z, E, h)$ une log-résolution de $(\bar{\Gamma}, \bar{\Gamma} \backslash \Gamma)$. Notons alors $i_{\bar{\Gamma}}$ l'immersion fermée $\bar{\Gamma} \rightarrow X \times_{\mathbb{P}_{k}^{1}} Y$ puis $h_{X}$ et $h_{Y}$ les composées $p_{X} \circ i_{\bar{\Gamma}} \circ h$ et $p_{Y} \circ i_{\bar{\Gamma}} \circ h$. Comme $f_{X}$ et $f_{Y}$ sont propres, le produit fibré est lui-même propre au dessus de $\mathbb{P}_{k}^{1}$, donc les projections sont propres. De plus, l'immersion fermée $i_{\bar{\Gamma}}$ est propre, donc par composition $h_{X}$ et $h_{Y}$ sont propres. Le graphe $\Gamma$ est isomorphe à $X \backslash F_{X}\left(\right.$ par $\left.p_{X} \circ i_{\bar{\Gamma} \mid \Gamma}\right)$, donc à $Y \backslash F_{Y}$ (par composition avec $\left.\Psi^{-1}\right)$. Ainsi $X \backslash F_{X}$ et $Y \backslash F_{Y}$ sont isomorphes à $(Z \backslash E)$ via $h_{X}$ et $h_{Y}$ et $\left(Z, E, h_{X}\right)$ (resp $\left.\left(Z, E, h_{Y}\right)\right)$ est une log-résolution de $\left(X, F_{X}\right)\left(\operatorname{resp}\left(Y, F_{Y}\right)\right)$. Enfin $i_{\bar{\Gamma}} \circ h$ étant à valeurs dans le produit fibré la composée $f_{X} \circ h_{X}$ est égale à $f_{Y} \circ h_{Y}$.

Prouvons 2.4. Soit $\left(X, i_{X}, f_{X}\right)$ et $\left(Y, i_{Y}, f_{Y}\right)$ deux compactifications de $f$. Rappelons que les fermés à l'infini $F_{X}$ et $F_{Y}$ contiennent les lieux singuliers $X_{\text {Sing }}$ et $Y_{\text {Sing }}$ ainsi que les fibres de l'infini $X_{\infty}$ et $Y_{\infty}$. Les ouverts $U_{X}$ et $U_{Y}$ sont isomorphes par l'isomorphisme $i_{X} \circ i_{Y}^{-1}$ que nous notons $\Psi$. Le morphisme $f_{Y}$ est alors la composée $f_{X} \circ \Psi$. Par le théorème de coiffage 2.8, il existe un quadruplet $\left(Z, E, h_{X}, h_{Y}\right)$ tel que la composée $f_{X} \circ h_{X}$ est égale à la composée $f_{Y} \circ h_{Y}$, le triplet $\left(Z, E, h_{X}\right)$ est une log-résolution du couple $\left(X, F_{X}\right)$ et le triplet $\left(Z, E, h_{Y}\right)$ est une log-résolution du couple $\left(Y, F_{Y}\right)$. Notons que $Z_{\infty}$, la fibre à l'infini des morphismes égaux $f_{X} \circ h_{X}$ et $f_{Y} \circ h_{Y}$, est un diviseur contenu 
dans $E$, donc naturellement à croisements normaux. On peut utiliser ces résolutions pour calculer et comparer les fibres de Milnor motiviques $S_{1 / f_{X}}\left(U_{X}^{*}\right)$ et $S_{1 / f_{Y}}\left(U_{Y}^{*}\right)$. On note $Z_{0}$ la fibre en zéro des morphismes égaux $f_{X} \circ h_{X}$ et $f_{Y} \circ h_{Y}$. Ainsi, le triplet $\left(Z \backslash Z_{0}, E \backslash Z_{0}, h_{X}\right)$ est une log-résolution du couple $\left(X \backslash X_{0}, F_{X} \backslash X_{0}\right)$ et $\left(Z \backslash Z_{0}, E \backslash Z_{0}, h_{Y}\right)$ est une log-résolution de $\left(Y \backslash Y_{0}, F_{Y} \backslash Y_{0}\right)$. Les ouverts $h_{X}^{-1}\left(U_{X}^{*}\right)$ et $h_{Y}^{-1}\left(U_{Y}^{*}\right)$ sont tous deux égaux à l'ouvert $(Z \backslash E) \backslash Z_{0}$. Par conséquent, par égalité des fonctions et des ouverts, $S_{1 / f_{X} \circ h_{X}}\left(h_{X}^{-1}\left(U_{X}^{*}\right)\right)$ est égale à $S_{1 / f_{Y} \circ h_{Y}}\left(h_{Y}^{-1}\left(U_{Y}^{*}\right)\right)$ dans l'anneau de Grothendieck $\mathcal{M}_{Z_{\infty} \times \mathbb{G}_{m}}^{\mathbb{G}_{m}}$. Par le théorème de rationalité 2.3 , en appliquant la composition des images directes $f_{X !} \circ h_{X !}$ et $f_{Y !} \circ h_{Y !}$, tous deux égaux à $\left(f_{X} \circ h_{X}\right)$ ! et $\left(f_{Y} \circ h_{Y}\right)$ ! , on obtient dans $\mathcal{M}_{\{\infty\} \times \mathbb{G}_{m}}^{\mathbb{G}_{m}}$ l'égalité voulue.

2.4. Réalisation de Hodge. - On suppose ici $k$ égal à $\mathbb{C}$. Soit $f: U \rightarrow \mathbb{A}_{\mathbb{C}}^{1}$ une application régulière à source lisse. Soit $(X, i, \hat{f})$ une compactification de

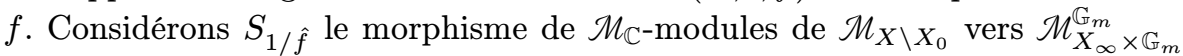
rappelé en 1.5. En appliquant le théorème 1.8 et la compatibilité des modules de Hodge mixtes avec l'image directe (ici sur le point) $R \hat{f}_{!}$nous obtenons avec les notations ci-dessus :

THÉORÈme 2.9. - Pour une compactification $(X, i, \hat{f})$, le diagramme suivant est commutatif :

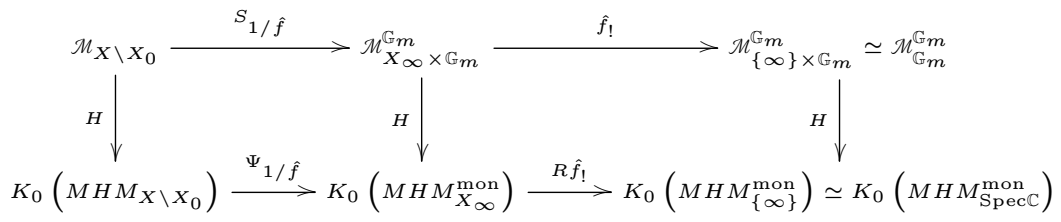

En particulier $H\left(S_{f, \infty}\right)=R \hat{f}_{!}\left(\Psi_{1 / \hat{f}}\left(R i_{!} \mathbf{Q}_{U}\right)\right)$.

Avec les notations de la partie 1.4.2 on déduit alors du théorème précédent ThÉORÈme 2.10. - Pour une application régulière $f: U \rightarrow \mathbb{A}_{\mathbb{C}}^{1}$, la classe de la structure de Hodge mixte limite de $f$ à l'infini est $\chi_{h}\left(S_{f, \infty}\right)$. En particulier, elle ne dépend pas de la compactification. Le spectre à l'infini de $f$ est alors égal à $\operatorname{Sp}\left(S_{f, \infty}\right)$.

Démonstration. - La structure de Hodge mixte limite sur $H_{c}^{k}\left(f^{-1}(t), \mathbb{Q}\right)$ s'identifie à la structure de Hodge mixte de $\mathbb{H}_{c}^{k}\left(\hat{f}^{-1}(\infty), \psi_{1 / \hat{f}}\left(R i_{!} \underline{\mathbb{Q}}_{U}\right)\right)$. On obtient ce groupe d'hypercohomologie en prenant le faisceau pervers sous jacent du module de Hodge mixte $R \hat{f}_{!}\left(\Psi_{1 / \hat{f}}\left(R i_{!} \mathbf{Q}_{U}\right)\right)$. La classe de la structure de Hodge mixte limite de $f$ à l'infini est par définition, la somme 
alternée $\sum_{k}(-1)^{k}\left[\mathbb{H}_{c}^{k}\left(\hat{f}^{-1}(\infty), \psi_{1 / \hat{f}}\left(R i_{\underline{\mathbb{Q}}} \underline{\underline{Q}}_{U}\right)\right)\right]$ dans $K_{0}\left(S H^{\text {mon }}\right)$. Elle vaut $\Phi\left(R \hat{f}_{!}\left(\Psi_{1 / \hat{f}}\left(R i_{!} \mathbf{Q}_{U}\right)\right)\right)$ égal à $\Phi\left(H\left(S_{f, \infty}\right)\right)$ par les paragraphes 1.4.2.2, 1.4.2.3 et le théorème 2.9 .

\section{Fibre de Milnor motivique à l'infini d'un polynôme non dégénéré}

3.1. Polyèdres de Newton et non dégénerescence. - Dans cette partie $k$ est un corps de caractéristique 0 et nous fixons un entier strictement positif $d$ et un polynôme de Laurent $f$ appartenant à l'anneau $k\left[x_{1}, x_{1}^{-1}, \ldots, x_{d}, x_{d}^{-1}\right]$. Nous noterons $\left(\alpha_{k}\right)_{k \in \mathbb{Z}}$ ses coefficients. Il définit une fonction $f: \mathcal{U} \rightarrow \mathbb{A}_{k}^{1}$ où $\mathcal{U}$ est son domaine de définition. Notons que $\mathcal{U}$ contient le tore $\mathbb{G}_{m}^{d}$. L'ensemble $\left\{\underline{k} \in \mathbb{Z}^{d} \mid \alpha_{\underline{k}} \neq 0\right\}$ est appelé support de $f$ et on le note $\operatorname{supp}(f)$. L'enveloppe convexe dans $\mathbb{R}^{d}$ de $\operatorname{supp}(f) \cup\{0\}$ est un polyèdre appelé polyèdre de Newton à l'infini de $f$ et noté $\Gamma_{-}$. On note $\Gamma$ les faces de $\Gamma_{-}$ne contenant pas l'origine. On notera enfin $\Gamma^{\circ}$ l'ensemble des faces de $\Gamma_{-}$qui ne sont pas contenues dans une face distincte de $\Gamma_{-}$et contenant 0 .

REMARQUe 3.1. - Cette définition du polyèdre de Newton correspond à celle de Kouchnirenko [22] pour les éléments de $k\left[x_{1}, \ldots, x_{d}\right]$ mais pas pour les polynômes de Laurent qui ne sont pas des polynômes. Nous justifierons ce choix à la remarque 3.7 .

Pour une face $\gamma$ de $\Gamma_{-}$on note $f_{\gamma}$ le polynôme $\sum_{\underline{k} \in \gamma} \alpha_{\underline{k}} \underline{x} \underline{\underline{k}}$. Au sens de Kouchnirenko [22], on dit que le polynôme $f$ est non dégénéré pour son polyèdre de Newton à l'infini, si pour toute face $\gamma$ de $\Gamma$ le polynôme $f_{\gamma}$ est lisse sur $\mathbb{G}_{m}^{d}$. Nous supposons dans toute la suite que le polynôme de Laurent $f$ est non dégénéré pour $\Gamma$.

Un polynôme $f$ dans $k\left[x_{1}, \ldots, x_{d}\right]$ est dit commode si et seulement si son polyèdre de Newton rencontre chaque axe de coordonnée. Un polynôme de Laurent $f$ dans $k\left[x_{1}, \ldots, x_{d}\right]\left[x_{1}^{-1}, \ldots, x_{d}^{-1}\right]$ et de polyèdre de Newton $\Gamma_{-}$est dit commode si et seulement si aucune face de $\Gamma_{-}$distincte de $\Gamma_{-}$ne contient 0 , autrement dit 0 appartient à l'intérieur du polyèdre $\Gamma_{-}$.

Enfin pour toute face $\gamma$ de $\Gamma$, on introduit la variété

$$
X_{\gamma}(0):=\left\{x \in \mathbb{G}_{m}^{d} \mid f_{\gamma}(x)=0\right\} .
$$

3.2. Compactification. - Il existe deux polynômes premiers entre eux $P$ et $Q$ appartenant à $k\left[x_{1}, \ldots, x_{d}\right]$ tels que $Q$ soit monomial et $f$ égal à la fraction rationnelle $P / Q$.

Notation 3.2. - Pour un polynôme de Laurent $\mathscr{P}$ en $d$ variables, on note $\tilde{\mathscr{P}}\left(\underline{x_{i, 0}, x_{i, 1}}\right)$ le polynôme homogène $\left(\prod_{i=1}^{d} x_{i, 0} \operatorname{deg}_{i} \mathscr{D}\right) \mathscr{P}\left(\underline{x_{i, 1} / x_{i, 0}}\right)$ où $\operatorname{deg}_{i} \mathscr{P}$ est le degré en la variable $x_{i}$. 
Dans $\left(\mathbb{P}^{1}\right)^{d} \times \mathbb{P}^{1}$, on considère alors la variété

$$
\begin{aligned}
& X:=\left\{\underline{\left(\left[x_{i, 0}: x_{i, 1}\right]\right.},[\alpha: \beta]\right) \\
&\left.\left|\alpha \tilde{P}\left(\underline{x_{i, 0}, x_{i, 1}}\right) \prod_{i=1}^{d} x_{i, 0} \operatorname{deg}_{i}(Q)=\beta \prod_{i=1}^{d} x_{i, 0}^{\operatorname{deg}_{i}(P)} \tilde{Q}\left(\underline{x_{i, 0}, x_{i, 1}}\right)\right|\right\}_{\text {red }},
\end{aligned}
$$

l'immersion ouverte dominante $i$ de $\mathcal{U}$ dans $X$ qui associe à $x$ de $\mathcal{U}$, l'élément $\left(\left[1: x_{i}\right],[1: f(x)]\right)$ et le morphisme propre $\hat{f}$ de $X$ vers $\mathbb{P}_{k}^{1}$ induit par la deuxième projection.

3.3. Résultats. - Nous commençons par traîter le cas d'un polynôme restreint au tore :

ThÉORÈme 3.3. - Pour un polynôme de Laurent $f: \mathbb{G}_{m}^{d} \rightarrow \mathbb{A}_{k}^{1}$ non dégénéré pour son polyèdre de Newton la fibre de Milnor motivique à l'infini est égale dans l'anneau $\mathcal{M}_{\mathbb{G}_{m}}^{\mathbb{G}_{m}} \grave{a}$

$$
S_{f, \infty}=-\sum_{\gamma \in \Gamma^{\circ}}(-1)^{d-\operatorname{dim} \gamma}\left[\mathbb{G}_{m}^{d} \backslash X_{\gamma}(0), f_{\gamma}^{-1}, \sigma_{\gamma}\right] .
$$

où $\Gamma^{\circ}$ est l'ensemble des faces du polyèdre de Newton non contenues dans les faces distinctes de $\Gamma_{-}$qui contiennent 0 , et pour toute face $\gamma, \sigma_{\gamma}$ est l'action du groupe $\mathbb{G}_{m}$ sur $X_{\gamma}(0)$ définie par $\sigma_{\gamma}\left(\lambda,\left(\underline{x_{i}}\right)\right)$ égal à $\left(\lambda^{-\omega_{i}} x_{i}\right)$ où $\omega$ est un élément du cône dual de la face $\gamma$.

EXEMPLE 3.4. - Si $f: \mathbb{G}_{m}^{d} \rightarrow \mathbb{A}_{k}^{1}$ est polynôme de Laurent non dégénéré et commode alors :

$$
S_{f, \infty}=-\sum_{\gamma \in \Gamma}(-1)^{d-\operatorname{dim} \gamma}\left[\mathbb{G}_{m}^{d} \backslash X_{\gamma}(0), f_{\gamma}^{-1}, \sigma_{\gamma}\right] .
$$

Si de plus ce polynôme de Laurent est un polynôme commode alors :

$$
S_{f, \infty}=-\sum_{\gamma \in \Gamma^{\circ}}(-1)^{d-\operatorname{dim} \gamma}\left[\mathbb{G}_{m}^{d} \backslash X_{\gamma}(0), f_{\gamma}^{-1}, \sigma_{\gamma}\right] .
$$

où $\Gamma^{\circ}$ est l'ensemble des faces du polyèdre de Newton qui ne sont pas contenues dans un hyperplan de coordonnées.

Nous traîtons ensuite le cas général des polynômes non dégénérés mais considérés sur leur ensemble de définition $\mathcal{U}$ (théorème 3.24). Par additivité du morphisme $S_{1 / \hat{f}}$ (théorème 1.5 ), on calcule $S_{1 / \hat{f}}(\mathcal{U})$ comme somme de $S_{1 / \hat{f}}$ évalué sur des sous tores de $\mathcal{U}$ puis on applique le théorème 3.3.

En particulier, par le corollaire 2.10 nous obtenons une décomposition du spectre à l'infini de $f$ en termes de spectres de variétés quasi-homogènes : 
THÉORÈme 3.5. - Lorsque $k$ est le corps des nombres complexes, si $f$ est un polynôme de Laurent non dégénéré pour son polyèdre de Newton alors le spectre à l'infini du morphisme $f: \mathbb{G}_{m}^{d} \rightarrow \mathbb{A}_{\mathbb{C}}^{1}$ est donné par la formule

$$
\operatorname{Sp}(f, \infty)=-\sum_{\gamma \in \Gamma^{\circ}}(-1)^{d-\operatorname{dim} \gamma} \operatorname{Sp}\left(\left[f_{\gamma}^{-1}(1), \sigma_{\hat{\mu}, \gamma}\right]\right)
$$

où $\Gamma^{\circ}$ est l'ensemble des faces du polyèdre de Newton, non contenues dans les faces distinctes de $\Gamma_{-}$qui contiennent 0 , et pour toute face $\gamma, \sigma_{\hat{\mu}, \gamma}$ est l'action du groupe $\hat{\mu}$ sur $f_{\gamma}^{-1}(1)$, définie par $\sigma_{\hat{\mu}, \gamma}\left(\lambda,\left(\underline{x_{i}}\right)\right)$ égal $\grave{a}\left(\lambda^{-\omega_{i}} x_{i}\right)$ où $\omega$ est un élément du cône dual de la face $\gamma$.

3.4. Calcul de la fibre de Milnor motivique à l'infini au dessus du tore. - Commençons par calculer la fibre de Milnor motivique de $f: \mathbb{G}_{m}^{d} \rightarrow \mathbb{A}_{k}^{1}$ (théorème 3.3). Dans cette sous-partie, nous notons U l'ouvert $i\left(\mathbb{G}_{m}^{d} \backslash f^{-1}(0)\right)$ de $X \backslash X_{0}$ et $F$ son complémentaire.

3.4.1. Arcs tracés sur $X$. - Les arcs tracés sur $X$ sont de la forme $\left(\left[x_{i, 0}(t): x_{i, 1}(t)\right],[\alpha(t): \beta(t)]\right)$ où $\alpha(t), \beta(t), x_{i, 0}(t)$ et $x_{i, 1}(t)$, pour tout $i$ appartenant $\{1, \ldots, d\}$, sont des séries formelles vérifiant dans l'anneau $k[[t]]$, l'équation

$$
\alpha(t) \underline{\tilde{P}}\left(\underline{\left.x_{i, 0}(t), x_{i, 1}(t)\right)} \prod_{i=1}^{d} x_{i, 0}(t)^{\operatorname{deg}_{i}(Q)}=\beta(t) \prod_{i=1}^{d} x_{i, 1}(t)^{\operatorname{deg}_{i}(P)} \underline{\tilde{Q}} \underline{\left(x_{i, 0}(t), x_{i, 1}(t)\right)}\right.
$$

et telles que les couples $(\alpha(0), \beta(0))$ et $\left(x_{i, 0}(0), x_{i, 1}(0)\right)$ pour tout $i$ appartenant $\{1, \ldots, d\}$, sont non nuls.

Remarque 3.6. - Dans toute la suite nous considérerons des arcs dont le point générique appartient à l'ouvert. Ces arcs ne sont donc pas tracés dans les hyperplans de coordonnées. Par conséquent, pour tout $i$ dans $\{1, \ldots, d\}$, les ordres $\operatorname{ord}_{t} x_{i, 0}(t)$ et $\operatorname{ord}_{t} x_{i, 1}(t)$ sont finis.

REMARQUE 3.7. - Tout arc $\varphi$, non tracé dans les hyperplans de coordonnées peut s'écrire sous la forme $\left(P_{i}(t) / t^{\omega_{i}}\right)$ où $\omega_{i}$ appartient à $\mathbb{Z}$ et $P_{i}(t)$ est une série formelle inversible pour tout $i$ dans $\{1, \ldots, d\}$. Dans toute la suite nous considérerons les arcs écrits sous cette forme. Notons $\alpha_{k}$ les coefficients de $f$. L'évaluation de $f$ sur un tel arc est :

$$
f(\varphi(t))=\sum_{k \in \operatorname{supp}(f)} \alpha_{k} P(t)^{k} t^{-(k \mid \omega)}
$$

où $P(t)^{k}$ est le produit $\prod_{i=1}^{d} P_{i}(t)^{k_{i}}$ et $(k \mid \omega)$ la somme $\sum_{i=1}^{d} k_{i} \omega_{i}$ pour tout $k$ dans $\operatorname{supp}(f)$. Pour calculer l'ordre de $f(\varphi(t))$, nous calculerons le maximum $\max \{(k \mid \omega) \mid k \in \operatorname{supp}(f)\}$ et nous considérerons les $\omega$ pour lesquels cette 
quantité est strictement positive. Nous serons donc amener à utiliser $\Gamma_{-}$l'enveloppe convexe de l'union $\operatorname{supp}(f) \cup\{0\}$. Pour tout $\omega$ dans $\mathbb{Z}^{d}$, notons $l_{\omega}$ la forme linéaire $\left(\omega \mid\right.$.) définie sur $\mathbb{R}^{d}$. Le maximum de la restriction $l_{\omega \mid \Gamma_{-}}$sera noté $m_{\Gamma}(\omega)$. Il est positif et atteint sur une face de $\Gamma_{-}$notée $\gamma(\omega)$. En particulier $\left(\omega \mid\right.$.) est constante sur $\gamma(\omega)$, car les lignes de niveaux de $l_{\omega}$ sont des hyperplans affines. Si ce maximum est strictement positif, il est alors atteint sur $\Gamma$, qui est l'ensemble des faces ne contenant pas 0 . S'il est nul alors il est atteint sur une face contenant 0 , or $\Gamma_{-}$contient le support de $f$, donc pour tout $k$ appartenant au support, le produit scalaire $(k \mid \omega)$ est négatif. On obtient alors :

Proposition et notations 3.8. - Soit $\omega$ dans $\mathbb{Z}^{d}$ et $\varphi$ un arc de Laurent de la forme $\left(P_{i}(t) / t^{\omega_{i}}\right)$ où chaque $P_{i}(t)$ est une série formelle inversible. On considère la forme linéaire $\left(\omega \mid\right.$.) définie sur $\mathbb{R}^{d}$. Ainsi :

1. Le maximum de $(\omega \mid$.$) restreinte à \Gamma_{-}$sera noté $m_{\Gamma}(\omega)$. Il est positif et atteint sur une face de $\Gamma_{-}$notée $\gamma(\omega)$. En particulier $(\omega \mid$.$) est constante$ sur $\gamma(\omega)$.

2. Notons $\left(\alpha_{\underline{k}}\right)_{\underline{k}}$ les coefficients du polynôme $f$, et posons pour toute face $\gamma$

$$
\tilde{f}_{\gamma}(\underline{x}, u):=f_{\gamma(\omega)}(\underline{x})+\sum_{\underline{k} \notin \gamma} \alpha_{\underline{k}} u^{m_{\Gamma}(\underline{\omega})-(\underline{k} \mid \omega)} \underline{x}^{\underline{k}} .
$$

On obtient alors l'égalité $f(\varphi(t))=\left(1 / t^{m_{\Gamma}(\omega)}\right) \tilde{f}_{\gamma(\omega)}\left(\underline{P_{i}}(t), t\right)$.

3. L'ordre $\operatorname{ord}_{t} 1 / f(\varphi(t))$ est égal à $m_{\Gamma}(\omega)-\operatorname{ord}_{t} \tilde{f}_{\gamma(\omega)}\left(\underline{P_{i}(0)}, t\right)$. En particulier si l'entier $\operatorname{ord}_{t} 1 / f(\varphi(t))$ est strictement positif alors $m_{\Gamma}(\omega)$ est strictement positif.

4. Lorsque $\left(P_{i}(0)\right)$ n'annule pas $f_{\gamma(\omega)}$, le coefficient angulaire ac $1 / f(\varphi(t))$ vaut $\left.1 / f_{\gamma(\omega)} \underline{\left(P_{i}(0)\right.}\right)$.

5. Pour tout arc $\overline{\varphi(t)}$ égal à $\left(P_{i}(t) / t^{\omega_{i}}\right)$, si l'origine $\varphi(0)$ appartient à $X_{\infty}$ alors l'ordre $\operatorname{ord}_{t} 1 / f(\varphi(t))$ est non nul donc $m_{\Gamma}(\omega)$ est strictement positif.

6. On note $\Omega$ l'ouvert $\left\{\omega \in \mathbb{Z}^{d} \mid m_{\Gamma}(\omega)>0\right\}$. Pour tout $\omega$ dans $\Omega$, la face $\gamma(\omega)$ ne contient pas 0 , elle appartient $\grave{a} \Gamma$.

7. Pour toute face $\gamma$ dans $\Gamma$, on désigne par $\sigma(\gamma)$ l'intérieur, dans son espace vectoriel engendré dans $\mathbb{R}^{d}$, du cône positif engendré par l'ensemble $\{\omega \in$ $\Omega \mid \gamma(\omega)=\gamma\}$. Cet ensemble est un cône polyédral rationnel convexe relativement ouvert. Pour une face $\gamma$ de la forme $\gamma_{1} \cap \cdots \cap \gamma_{s}$ intersections de faces de codimension 1 , en notant $\alpha^{(i)}$ le vecteur normal à $\gamma_{i}$, extérieur $\grave{a} \Gamma$, à coordonnées entières et de plus petite norme, le cône $\sigma(\gamma)$ est égal $\grave{a}$ la somme $\sum_{i=1}^{s} \mathbb{R}_{*}^{+} \alpha^{(i)}$ de dimension $d-\operatorname{dim} \gamma$. 
Démonstration. - Tout est immédiat et classique. Le polyèdre de Newton $\Gamma_{-}$ est compact, la forme linéaire $(\omega \mid$.) est continue et s'annule en 0. Par conséquent le maximum de la restriction est positif. Les lignes de niveaux sont des hyperplans, ce maximum est donc atteint sur une face de $\Gamma_{-}$.

ExemPles 3.9. - Quelques exemples :

1. Soit $\gamma$ une face de $\Gamma_{-}$de codimension 1 et $\vec{n}$ dans $\mathbb{Z}^{d}$ le vecteur normal à $\gamma$ extérieur à $\Gamma_{-}$et de norme minimale. En ce cas, la face $\gamma(\vec{n})$ est la face $\gamma$.

2. Pour un polynôme commode l'ouvert $\Omega$ est égal à $\mathbb{Z}^{d} \backslash \mathbb{Z}_{-}^{d}$.

3. Pour un polynôme de Laurent commode l'ouvert $\Omega$ est égal à $\mathbb{Z}^{d}$.

\subsubsection{Fonction zêta motivique}

DÉfinition 3.10. - Soit $n$ et $\delta$ deux entiers strictement positifs, on pose

$$
\mathscr{X}_{n, U}^{\delta}(1 / \hat{f})=\left\{\begin{array}{l|l}
\varphi \in L\left(X \backslash X_{0}\right) & \begin{array}{l}
\operatorname{ord}_{t} F \varphi \leq n \delta \\
\operatorname{ord}_{t} 1 / \hat{f}(\varphi(t))=n
\end{array}
\end{array}\right\} .
$$

On munit $\mathscr{X}_{n, U}^{\delta}(1 / \hat{f})$ de la flèche $\left(\pi_{0}, a c\right)$, « (origine, coefficient angulaire) » vers $X_{\infty} \times \mathbb{G}_{m}$ qui a un $\operatorname{arc} \varphi$ associe $(\varphi(0)$, ac $1 / \hat{f}(\varphi))$, et de l'action $\sigma$ de $\mathbb{G}_{m}$. On vérifie que les fibres du morphisme $\pi_{0}$ sont invariantes sous l'action de $\sigma$ et $a c$ est homogène de poids $n$ pour $\sigma$. Pour tout entier positif $k$, les tronqués $\left(\pi_{n \delta+k}\left(\mathscr{X}_{n, U}^{\delta}(1 / \hat{f})\right),\left(\pi_{n \delta+k, 0}, a c\right), \sigma\right)$ appartiennent à $\operatorname{Var}_{X \infty \times \mathbb{G}_{m}}^{\mathbb{G}_{m}}$.

Proposition 3.11. - Pour tout couple d'entiers strictement positifs $(n, \delta)$, $\mathscr{X}_{n, U}^{\delta}(1 / \hat{f})$ se réécrit comme suit :

$$
\mathscr{X}_{n, U}^{\delta}(1 / \hat{f})=\left\{\begin{array}{l|l}
\varphi(t)=\left(P_{i}(t) / t^{\omega_{i}}\right) & \begin{array}{l}
P_{i}(t) \in k[[t]]^{*} \\
\left(\omega_{i}\right) \in \Omega \\
\forall i \in\{1, \ldots, d\}\left|\omega_{i}\right| \leq n \delta \\
\operatorname{ord}_{t} 1 / f\left(\underline{P_{i}(t) / t^{\omega_{i}}}\right)=n
\end{array}
\end{array}\right\} .
$$

Démonstration. - Il suffit de remarquer que le fermé $F$ est localement donné par les équations $x_{i, 0}=0$ et $x_{i, 1}=0$ avec $i$ appartenant à $\{1, \ldots, d\}$.

Par la définition 1.3, pour tout entier $\delta$ strictement positif, on considère la fonction zêta motivique modifiée

$$
Z_{1 / \hat{f}, U}^{\delta}(T):=\sum_{n=1}^{\infty} \mu\left(\mathscr{X}_{n, U}^{\delta}(1 / \hat{f})\right) T^{n} \in \mathcal{M}_{X_{\infty} \times \mathbb{G}_{m}}^{\mathbb{G}_{m}}[[T]]
$$

Proposition 3.12. - Décomposition de la fonction zêta motivique: 
- Pour tout couple d'entiers strictement positifs $(n, \delta)$, pour tout $\left(\omega_{i}\right)$ dans $\Omega$, on note

$$
\mathscr{X}_{n, \omega}^{\delta}(f):=\left\{\left(P_{i}(t) / t^{a_{i}}\right) \in \mathscr{X}_{n, U}^{\delta}(f) \mid \omega_{i}=a_{i}\right\} .
$$

Cet espace d'arcs est muni de l'application « origine, coefficient angulaire » et de l'action de $\mathbb{G}_{m}$ induites par celle de $\mathscr{X}_{n, U}^{\delta}(f)$.

- Pour une face $\gamma$ fixée, pour tout entier $\delta$ strictement positif, nous considérons les cônes

$$
C_{\gamma}^{\delta,=}:=\left\{\omega \in \sigma(\gamma)|| \omega_{i} \mid \leq m_{\Gamma}(\omega) \delta\right\}
$$

et

$$
C_{\gamma}^{\delta,<}:=\left\{n \in \mathbb{N}^{*}, \omega \in \sigma(\gamma)\left|\forall i \in\{1, \ldots, d\} m_{\Gamma}(\omega)>n \geq\right| \omega_{i} / \delta \mid\right\} .
$$

Tous deux sont des cônes polyédraux rationnels convexes.

Avec ces notations pour tout entier $\delta$ strictement positif, la fonction zêta se décompose comme suit :

$$
\begin{aligned}
Z_{1 / \hat{f}, U}^{\delta}(T)=\sum_{\gamma \in \Gamma} \sum_{\omega \in C_{\gamma}^{\delta,=} \cap \mathbb{Z}^{d}} \mu\left(\mathscr{X}_{m_{\Gamma}(\omega), \omega}^{\delta}(f)\right) T^{m_{\Gamma}(\omega)} & \\
& +\sum_{\gamma \in \Gamma} \sum_{(n, \omega) \in C_{\gamma}^{\delta,<} \cap \mathbb{Z}^{d+1}} \mu\left(\mathscr{X}_{n, \omega}^{\delta}(f)\right) T^{n} .
\end{aligned}
$$

Démonstration. - Il suffit de classer les arcs suivant leur $\omega$, puis chaque $\omega$ suivant la face $\gamma(\omega)$ qui lui est associée et enfin d'appliquer l'additivité de la mesure.

3.4.3. Stratification des cônes. - On désigne par $\mathcal{E}$ l'ensemble $\{-1,0,1\}^{d}$. Par la suite, à cause de l'action de $\mathbb{G}_{m}$, nous aurons besoin de stratifier :

- $\mathbb{R}^{d}$ en cônes de la forme $C_{\varepsilon}$ où $\varepsilon$ appartient à $\mathcal{E}$ et

$$
C_{\varepsilon}:=\left\{\left(k_{i}\right) \in \mathbb{R}^{d} \mid k_{i}>0 \text { si } \varepsilon_{i}>0, k_{i}<0 \text { si } \varepsilon_{i}<0, k_{i}=0 \text { si } \varepsilon_{i}=0\right\} .
$$

Chaque $C_{\varepsilon}$ est un «quadrant » de $\mathbb{R}^{d}$.

- $\sigma(\gamma)$ en cônes $\sigma_{\gamma, \varepsilon}$ égal à $\sigma(\gamma) \cap C_{\varepsilon}$, pour toute face $\gamma$ de $\Gamma$ et $\varepsilon$ dans $\mathcal{E}$.

- $C_{\gamma}^{\delta,=}$ en cônes $C_{\gamma, \varepsilon}^{\delta,=}$ égal à $C_{\gamma}^{\delta,=} \cap C_{\varepsilon}$ et $C_{\gamma}^{\delta,<}$ en cônes $C_{\gamma, \varepsilon}^{\delta,<}$ de la forme $C^{\delta,<\cap} C_{\varepsilon}$, pour une face $\gamma$ fixée, pour tout entier $\delta$ strictement positif et $\varepsilon$ dans $\mathcal{E}$. 
3.4.4. L'élément $\mathbb{G}_{m}^{d} \backslash X_{\gamma}(0)$ vu dans $\operatorname{Var}_{X_{\infty} \times \mathbb{G}_{m}}^{\mathbb{G}_{m}}$. - Soit $\gamma$ une face de $\Gamma$ et $\omega$ dans $\sigma(\gamma)$.

Avec les notations 2.1.1, on munit $\mathbb{G}_{m}^{d} \backslash X_{\gamma}(0)$ de la flèche $p_{\omega}$ vers $X_{\infty} \times \mathbb{G}_{m}$

$$
p_{\omega}:\left(\underline{a_{i}}\right) \mapsto\left(\left(\left[\underline{x_{i}}\right],[0: 1]\right), 1 / f_{\gamma}\left(\underline{a_{i}}\right)\right)
$$

où $x_{i}$ vaut l'infini si $\omega_{i}$ est strictement positif, 0 si $\omega_{i}$ est strictement négatif et $a_{i}$ si $\omega_{i}$ est nul. Remarquons que $\left(\left[x_{i}\right],[0: 1]\right)$ appartient bien à $X_{\infty}$; par exemple comme origine de l'arc $\left(a_{i} / t^{\omega_{i}}\right)$ de $\mathscr{X}_{m_{\Gamma}(\omega), \omega}^{\delta}$. Pour $\omega^{\prime}$ et $\omega^{\prime \prime}$ appartenant au cone $\sigma(\gamma)$, les applications $p_{\omega^{\prime}}$ et $p_{\omega^{\prime \prime}}$ sont égales si et seulement si pour tout $i$ dans $\{1, \ldots, d\}$, les entiers $\omega_{i}$ et $\omega_{i}^{\prime}$ sont tous les deux nuls, ou ont le même signe. Soit $\omega$ dans $\sigma(\gamma)$, l'application $p_{\omega}$ ne dépend donc que de la strate $C_{\varepsilon}$ contenant $\omega$, on note donc $p_{\gamma, \varepsilon}$ cette application.

On munit de plus $\mathbb{G}_{m}^{d} \backslash X_{\gamma}(0)$ de l'action $\sigma_{\omega}$ du groupe $\mathbb{G}_{m}$ :

$$
\sigma_{\omega}\left(\lambda,\left(\underline{x_{i}}\right)\right)=\left(\underline{\lambda^{-\omega_{i}} x_{i}}\right) .
$$

Notons que par quasi-homogénéité de $f_{\gamma}$, la flèche vers $\mathbb{G}_{m}$ est homogène de poids $m_{\Gamma}(\omega)$. Par opposition à $p_{\gamma, \varepsilon}$ cette action dépend de $\omega$. On obtient alors la variété $\left(\mathbb{G}_{m}^{d} \backslash X_{\gamma}(0) \stackrel{p_{\gamma, \varepsilon}}{\rightarrow} X_{\infty} \times \mathbb{G}_{m}, \sigma_{\omega}\right)$ appartenant à $\operatorname{Var}_{X_{\infty} \times \mathbb{G}_{m}}^{\mathbb{G}_{m}}$. Néanmoins on dispose de la proposition

Proposition 3.13. - Pour une face $\gamma$ dans $\Gamma$, pour $\varepsilon$ dans $\mathcal{E}$, pour $\omega$ et $\omega^{\prime}$ appartenant au cône $\sigma_{\gamma, \varepsilon}$, les classes sont classes sont égales dans l'anneau $\mathcal{M}_{X_{\infty} \times \mathbb{G}_{m}}^{\mathbb{G}_{m}}$

$$
\left[\mathbb{G}_{m}^{d} \backslash X_{\gamma}(0) \stackrel{p_{\gamma, \varepsilon}}{\rightarrow} X_{\infty} \times \mathbb{G}_{m}, \sigma_{\omega}\right]=\left[\mathbb{G}_{m}^{d} \backslash X_{\gamma}(0) \stackrel{p_{\gamma, \varepsilon}}{\rightarrow} X_{\infty} \times \mathbb{G}_{m}, \sigma_{\omega^{\prime}}\right] .
$$

On notera cette classe $\left[\mathbb{G}_{m}^{d} \backslash X_{\gamma}(0) \stackrel{p_{\gamma, \varepsilon}}{\rightarrow} X_{\infty} \times \mathbb{G}_{m}, \sigma_{\gamma}\right]$. De plus, cette classe $\left[\mathbb{G}_{m}^{d} \backslash X_{\gamma}(0) \stackrel{\pi_{\mathbb{G}_{m}} \circ p_{\gamma, \varepsilon}}{\rightarrow} \mathbb{G}_{m}, \sigma_{\gamma}\right]$ ne dépend pas de la strate $\sigma_{\gamma, \varepsilon}$, on la notera $\left[\mathbb{G}_{m}^{d} \backslash X_{\gamma}(0) \stackrel{f_{\gamma}^{-1}}{\rightarrow} \mathbb{G}_{m}, \sigma_{\gamma}\right]$.

Démonstration. - Soit $\omega$ et $\omega^{\prime}$ appartenant au cône $\sigma_{\gamma, \varepsilon}$, montrons l'égalité $\left[\mathbb{G}_{m}^{d} \backslash X_{\gamma}(0) \stackrel{p_{\gamma, \varepsilon}}{\rightarrow} X_{\infty} \times \mathbb{G}_{m}, \sigma_{\omega}\right]=\left[\mathbb{G}_{m}^{d} \backslash X_{\gamma}(0) \stackrel{p_{\gamma, \varepsilon}}{\rightarrow} X_{\infty} \times \mathbb{G}_{m}, \sigma_{\omega^{\prime}}\right] \in \mathcal{M}_{X_{\infty} \times \mathbb{G}_{m}}^{\mathbb{G}_{m}}$.

Dans ce qui suit nous sous entendrons le but $X_{\infty} \times \mathbb{G}_{m}$ dans l'écriture des classes. Notons $\langle\gamma\rangle$ le sous réseau saturé engendré par $\gamma$. On note $r$ son rang. Par application du théorème de la base adaptée il existe une base $\left(e_{1}, \ldots, e_{d}\right)$ de $\mathbb{Z}^{d}$ telle que $\left(e_{1}, \ldots, e_{r}\right)$ soit une base de $\langle\gamma\rangle$. Les facteurs invariants sont égaux à 1 car $\langle\gamma\rangle$ est saturé. Notons $A=\left(a_{i}^{j}\right)$ ( $i$ indiçant les lignes et $j$ les colonnes) la matrice de passage de la base $\left(e_{i}\right)$ à la base canonique de $\mathbb{Z}^{d}$; les colonnes de $A$ sont les vecteurs de la base canonique exprimés dans 
la base $\left(e_{i}\right)$. En particulier, pour tout $\beta$ appartenant à $\gamma, A \beta$ est de la forme ${ }^{t}\left(\delta_{1}, \ldots, \delta_{r}, 0, \ldots, 0\right)$. Considérons alors l'isomorphisme $\Phi$ induit sur $\mathbb{G}_{m}^{d}$ :

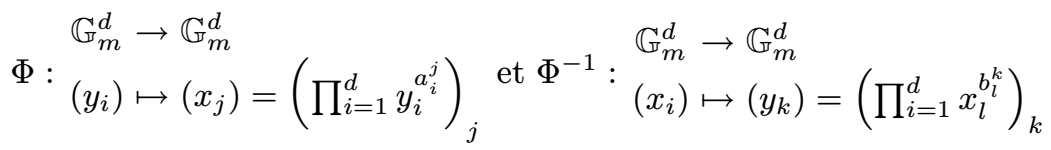

Pour $y$ dans $\mathbb{G}_{m}^{d}$ et $\omega$ dans $\mathbb{Z}^{d}$ on note $y^{\alpha}$ le produit $\prod_{i=1}^{d} y_{i}^{\alpha_{i}}$. Le produit $\Phi(y)^{\beta}$ est égal à $y^{A \beta}$ pour $\beta$ appartenant à $\gamma$. Par conséquent, pour tout $\beta$ appartenant à $\gamma, \Phi(y)^{\beta}$ est un monôme en les variables $y_{1}, \ldots, y_{r}$, donc le polynôme $f_{\gamma} \circ \Phi$ est lui même un polynôme en les variables $y_{1}, \ldots, y_{r}$, il s'écrit $\sum_{\beta \in \gamma} c_{\beta} y^{A \beta}$ pour $f_{\gamma}(x)$ égal à $\sum_{\beta \in \gamma} c_{\beta} x^{\beta}$. Notons alors

$$
g_{\gamma}: \begin{gathered}
\mathbb{G}_{m}^{r} \\
\left(y_{1}, \ldots, y_{r}\right)
\end{gathered} \underset{\left(f_{\gamma} \circ \Phi\right)\left(y_{1}, \ldots, y_{r}, 1, \ldots, 1\right)}{\mathbb{G}_{m}} .
$$

et considérons le diagramme commutatif :

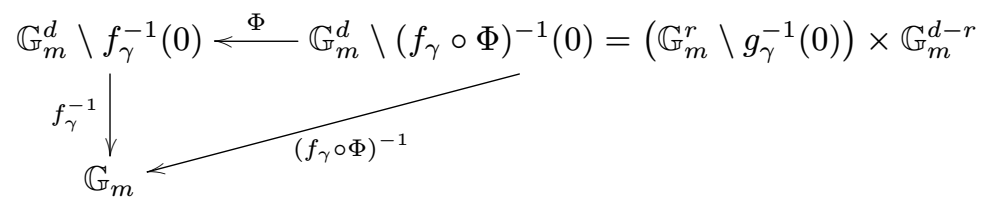

Par l'isomorphisme $\Phi$, l'action $\sigma_{\omega}$ de $\mathbb{G}_{m}$ sur $\mathbb{G}_{m}^{d} \backslash f_{\gamma}^{-1}(0)$ est transformée en l'action $\sigma_{t} A^{-1} \omega$ de $\mathbb{G}_{m}$ sur $\mathbb{G}_{m}^{d} \backslash\left(f_{\gamma} \circ \Phi\right)^{-1}(0)$. En effet, notons $\tilde{\sigma_{\omega}}$ l'action remontée par $\Phi$. Pour tout $\lambda$ dans $\mathbb{G}_{m}$, pour tout $x$ dans $\mathbb{G}_{m}^{d}$, l'action $\tilde{\sigma_{\omega}}$ vérifie nécessairement l'égalité entre $\Phi\left(\tilde{\sigma_{\omega}}\left(\lambda, \underline{x_{i}}\right)\right)$ et $\sigma_{\omega}\left(\lambda, \Phi\left(\underline{x_{i}}\right)\right)$, ou encore entre $\tilde{\sigma_{\omega}}\left(\lambda, \underline{x_{i}}\right)$ et $\Phi^{-1}\left(\sigma_{\omega}\left(\lambda, \Phi\left(\underline{x_{i}}\right)\right)\right.$. On obtient ainsi les égalités :

$$
\begin{aligned}
& \tilde{\sigma_{\omega}}(\lambda, x)=\left(\prod_{l=1}^{d}\left(\lambda^{-\omega_{l}} \prod_{i=1}^{d} x_{i}^{a_{i}^{l}}\right)^{b_{l}^{k}}\right)_{k} \\
& =\left(\lambda^{-\sum_{l=1}^{d} \omega_{l} b_{l}^{k}} \prod_{i=1}^{d} x_{i}^{\sum_{i=1}^{d} a_{i}^{l} b_{l}^{k}}\right)_{k}=\left(\lambda^{-\sum_{l=1}^{d} \omega_{l} b_{l}^{k}} x_{k}\right)_{k}=\lambda^{-t} A^{-1} \omega x .
\end{aligned}
$$

Montrons que pour tout $y$ dans $\mathbb{G}_{m}^{d} \backslash\left(f_{\gamma} \circ \Phi\right)^{-1}(0)$ et pour tout $\lambda$ dans $\mathbb{G}_{m}, \tilde{\sigma_{\omega}}(\lambda, y)$ n'appartient pas à $\left(f_{\gamma} \circ \Phi\right)^{-1}(0)$. Sinon, par application de $\Phi$, $\sigma_{\omega}(\lambda, \Phi(y))$ appartient à $f_{\gamma}^{-1}(0)$. Or pour tout $\lambda$ dans $\mathbb{G}_{m}$, pour tout $x$ dans $\mathbb{G}_{m}^{d}, \sigma_{\omega}(\lambda, x)$ appartient à $f_{\gamma}^{-1}(0)$ si et seulement si $x$ appartient à $f_{\gamma}^{-1}(0)$ car $f_{\gamma}\left(\sigma_{\omega}(\lambda, x)\right)$ est égal à̀ $\lambda^{-m_{\Gamma}(\omega)} f_{\gamma}(x)$. Donc $\Phi(y)$ appartient à $f_{\gamma}^{-1}(0)$ donc $y$ 
appartient à $\left(f_{\gamma} \circ \Phi\right)^{-1}(0)$. Contradiction. Par isomorphisme, nous avons dans $\mathcal{M}_{X_{\infty} \times \mathbb{G}_{m}}^{\mathbb{G}_{m}}$ l'égalité des classes

$$
\left[\mathbb{G}_{m}^{d} \backslash X_{\gamma}(0), p_{\gamma, \varepsilon}, \sigma_{\omega}\right]=\left[\left(\mathbb{G}_{m}^{r} \backslash g_{\gamma}^{-1}(0)\right) \times \mathbb{G}_{m}^{d-r}, \tilde{p}_{\sigma_{\gamma, \varepsilon}}, \tilde{\sigma_{\omega}}\right]
$$

où $\tilde{p}_{\sigma_{\gamma, \varepsilon}}$ est égal à $\left(\pi_{X_{\infty}} \circ p_{\gamma, \varepsilon},\left(f_{\gamma} \circ \Phi\right)^{-1}\right)$ et $\tilde{\sigma_{\omega}}$ est égal à $\sigma_{t} A^{-1} \omega$. Pour montrer que dans l'anneau $\mathcal{M}_{X_{\infty} \times \mathbb{G}_{m}}^{\mathbb{G}_{m}}$, la classe $\left[\mathbb{G}_{m}^{d} \backslash X_{\gamma}(0), p_{\gamma, \varepsilon}, \sigma_{\omega}\right]$ est égale à la classe $\left[\mathbb{G}_{m}^{d} \backslash X_{\gamma}(0), p_{\gamma, \varepsilon}, \sigma_{\omega^{\prime}}\right]$ nous montrons plutôt l'égalité entre les classes $\left[\left(\mathbb{G}_{m}^{r} \backslash g_{\gamma}^{-1}(0)\right) \times \mathbb{G}_{m}^{d-r}, \tilde{p}_{\sigma_{\gamma, \varepsilon}}, \tilde{\sigma_{\omega}}\right]$ et $\left[\left(\mathbb{G}_{m}^{r} \backslash g_{\gamma}^{-1}(0)\right) \times \mathbb{G}_{m}^{d-r}, \tilde{p}_{\sigma_{\gamma, \varepsilon}},{\tilde{\sigma^{\prime}}}^{\prime}\right]$. Notons pour cela $\tilde{\omega}$ le vecteur ${ }^{t} A^{-1} \omega$. Pour tout $y$ appartenant à $\left(\mathbb{G}_{m}^{r} \backslash g_{\gamma}^{-1}(0)\right) \times \mathbb{G}_{m}^{d-r}$ et pour tout $\lambda$ dans $\mathbb{G}_{m}, \tilde{\sigma_{\omega}}(\lambda, y)$ est égal à $\left(\lambda^{-\tilde{\omega}_{i}} y_{i}\right)$. On décompose cette action en un produit $\tilde{\sigma_{\omega}}=\sigma_{\tilde{\omega}}=\sigma_{\pi_{\mathbb{Z}^{r}} \tilde{\omega}} \times \sigma_{\pi_{\mathbb{Z}^{d-r}} \tilde{\omega}}$. La fonction $f_{\gamma} \circ \Phi$ ne dépend que des variables $y_{1}, \ldots, y_{r}$, et nous obtenons le diagramme

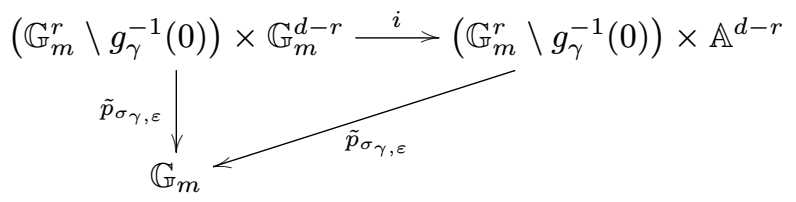

où $i$ est l'injection canonique. L'action $\sigma_{\tilde{\omega}}$ se prolonge naturellement à $\left(\mathbb{G}_{m}^{r} \backslash\right.$ $\left.g_{\gamma}^{-1}(0)\right) \times \mathbb{A}^{d-r}$. Nous sommes dans le cas d'application de l'identification des actions 1.1.2, ainsi la classe $\left[\left(\mathbb{G}_{m}^{r} \backslash g_{\gamma}^{-1}(0)\right) \times \mathbb{A}^{d-r}, \tilde{p}_{\sigma_{\gamma, \varepsilon}}, \sigma_{\tilde{\omega}}=\sigma_{\pi_{\mathbb{Z}^{r} \tilde{\omega}}} \times \sigma_{\pi_{\mathbb{Z}^{d-r}} \tilde{\omega}}\right]$ ne dépend pas de l'action $\sigma_{\pi_{\mathbb{Z}^{d-r}} \tilde{\omega}}$ de $\mathbb{G}_{m}$ sur $\mathbb{A}^{d-r}$. En effet, l'action $\sigma_{\tilde{\omega}}$ relève l'action $\sigma_{\pi_{\mathbb{Z}^{r}}}$ de $\mathbb{G}_{m}$ sur la variété $\mathbb{G}_{m}^{r} \backslash g_{\gamma}^{-1}(0)$ et cela, quelque soit l'action $\sigma_{\pi_{\mathbb{Z}^{d-r}} \tilde{\omega}}$ de $\mathbb{G}_{m}$ sur $\mathbb{A}^{d-r}$. Cette identification se propage alors à la classe $\left[\left(\mathbb{G}_{m}^{r} \backslash g_{\gamma}^{-1}(0)\right) \times \mathbb{G}_{m}^{d-r}, \tilde{p}_{\sigma_{\gamma, \varepsilon}}, \tilde{\sigma_{\omega}}\right]$.

LeMme 3.14. - Si $\left(Y \times \mathbb{G}_{m}^{l}, p, \sigma_{Y} \times \sigma_{\mathbb{G}_{m}^{l}}\right)$ et $\left(Y \times \mathbb{G}_{m}^{l}, p, \sigma_{Y} \times \sigma_{\mathbb{G}_{m}^{l}}^{\prime}\right)$ admettent un prolongement naturel en $\left(Y \times \mathbb{A}^{l}, p, \sigma_{Y} \times \sigma_{\mathbb{G}_{m}^{l}}\right)$ et $\left(Y \times \mathbb{G}_{m}^{l}, p, \sigma_{Y} \times \sigma_{\mathbb{G}_{m}^{l}}^{\prime}\right)$ alors les classes $\left[Y \times \mathbb{G}_{m}^{l}, p, \sigma_{Y} \times \sigma_{\mathbb{G}_{m}^{l}}\right]$ et $\left[Y \times \mathbb{G}_{m}^{l}, p, \sigma_{Y} \times \sigma_{\mathbb{G}_{m}^{l}}^{\prime}\right]$ sont égales.

Démonstration. - La preuve se fait par récurence sur $l$, en utilisant les relations d'additivités, l'égalité des classes $\left[Y \times\{0\}^{k} \times \mathbb{A}^{l-k}, p, \sigma_{Y} \times \sigma_{\mathbb{G}_{m}^{l}}\right]$ et $\left[Y \times\{0\}^{k} \times \mathbb{A}^{l-k}, p, \sigma_{Y} \times \sigma_{\mathbb{G}_{m}^{l}}^{\prime}\right]$, la stratification de $\mathbb{A}_{k}^{d}$ en produit de tores et enfin l'hypothèse de récurence sur les variétés qui sont du type suivant $\left(Y \times\{0\}^{k} \times \mathbb{G}_{m}^{l-k}, p, \sigma_{Y} \times \sigma_{\mathbb{G}_{m}^{l}}\right)$. Le point de départ étant l'égalité entre les classes $\left[Y \times\{0\}^{l}, p, \sigma_{Y} \times \sigma_{\mathbb{G}_{m}^{l}}\right]$ et $\left[Y \times\{0\}^{l}, p, \sigma_{Y} \times \sigma_{\mathbb{G}_{m}^{l}}^{\prime}\right]$.

On obtient ainsi l'égalité

$$
\begin{aligned}
{\left[\left(\mathbb{G}_{m}^{r} \backslash g_{\gamma}^{-1}(0)\right) \times \mathbb{G}_{m}^{d-r},\right.} & \left.\tilde{p}_{\sigma_{\gamma, \varepsilon}}, \sigma_{\pi_{\mathbb{Z}^{r}} \tilde{\omega}} \times \sigma_{\pi_{\mathbb{Z}^{d-r}} \tilde{\omega}}\right] \\
& =\left[\left(\mathbb{G}_{m}^{r} \backslash g_{\gamma}^{-1}(0)\right), \tilde{p}_{\sigma_{\gamma, \varepsilon}}, \sigma_{\pi_{\mathbb{Z}^{r}} \tilde{\omega}}\right] *(\mathbb{L}-1)^{d-r g(<\gamma>)} .
\end{aligned}
$$


Montrons alors que $\left[\left(\mathbb{G}_{m}^{r} \backslash g_{\gamma}^{-1}(0)\right), \tilde{p}_{\sigma_{\gamma, \varepsilon}}, \sigma_{\pi_{\mathbb{Z}^{r}} \tilde{\omega}}\right]$ et $\left[\left(\mathbb{G}_{m}^{r} \backslash g_{\gamma}^{-1}(0)\right), \tilde{p}_{\sigma_{\gamma, \varepsilon}}, \sigma_{\pi_{\mathbb{Z}^{r}} \tilde{\omega}^{\prime}}\right]$ sont égaux. Par adjonction, pour tout $\beta$ dans $\gamma,\left(\pi_{\mathbb{Z}^{r}}\left({ }^{t} A^{-1} \omega\right) \mid \pi_{\mathbb{Z}^{r}}(A \beta)\right)$, $\left({ }^{t} A^{-1} \omega \mid A \beta\right)$ et $(\omega \mid \beta)$ sont égaux car $\pi_{\mathbb{Z}^{d-r}}(\beta)$ est nul. La face $\gamma$ ne contient pas 0 donc $(\omega \mid \beta)$ est égal à $m_{\Gamma}(\omega)$ et donc strictement positif ainsi $\pi_{\mathbb{Z}^{r}}\left({ }^{t} A^{-1} \omega\right)$ est non nul. Les vecteurs $\pi_{\mathbb{Z}^{r}}\left({ }^{t} A^{-1} \omega\right)$ et $\pi_{\mathbb{Z}^{r}}\left({ }^{t} A^{-1} \omega^{\prime}\right)$ sont alors non nuls. Ces vecteurs sont colinéaires dans $\mathbb{Z}^{r}$ : les formes linéaires $\left(\pi_{\mathbb{Z}^{r}}\left({ }^{t} A^{-1} \omega\right) \mid.\right)$ et $\left(\pi_{\mathbb{Z}^{r}}\left({ }^{t} A^{-1} \omega\right) \mid.\right)$ sont constantes sur l'hyperface $A \gamma$ car $\gamma$ ne contient pas 0 . Elles sont donc nulles sur la direction $A \vec{\gamma}$ qui est un hyperplan de $\mathbb{R}^{r}$, les vecteurs sont normaux à cette hyperplan et donc colinéaires de rapports $k$. Le passage de $\sigma_{\omega}$ à $\sigma_{\omega^{\prime}}$ se fait alors par ramification puis par identification des actions 1.1.2. Les résultats suivent en remarquant que par définition $f_{\gamma}^{-1}$ est $\pi_{\mathbb{G}_{m}} \circ p_{\sigma, \varepsilon_{i}}$.

3.4.5. L'élément $X_{\gamma}(0) \times \mathbb{G}_{m}$ vu dans $\operatorname{Var}_{X_{\infty} \times \mathbb{G}_{m}}^{\mathbb{G}_{m}} \cdot$ - On munit $X_{\gamma}(0) \times \mathbb{G}_{m}$ de la flèche $p_{\omega}$ vers $X_{\infty} \times \mathbb{G}_{m}$ :

$$
p_{\omega}:\left(\left(a_{i}\right), \mu\right) \mapsto\left(\left(\underline{\left[x_{i}\right]},[0: 1]\right), \mu\right)
$$

où $x_{i}$ vaut l'infini si $\omega_{i}$ est strictement positif, 0 si $\omega_{i}$ est strictement négatif et $a_{i}$ si $\omega_{i}$ est nul. Remarquons que $\left(\left[x_{i}\right],[0: 1]\right)$ appartient bien à $X_{\infty}$, par exemple comme origine de l'arc $\left(a_{i} / t^{\omega_{i}}\right)$ de $\mathscr{X}_{m, \omega}^{\delta}$, où par la proposition 3.8, $m$ appartient à $\left\{1, \ldots, m_{\Gamma}(\omega)-1\right\}$. Comme dans 3.4.4, la fonction $p_{\omega}$ ne dépend que de $\sigma_{\gamma, \varepsilon}$, on la note donc $p_{\gamma, \varepsilon}$. On munit de plus $X_{\gamma}(0) \times \mathbb{G}_{m}$ de l'action $\tau_{n}$ de $\mathbb{G}_{m}$ définie par $\tau_{n}\left(\lambda,\left(\left(a_{i}\right), \mu\right)\right)$ égal à $\left(\left(a_{i}\right), \lambda^{n} \mu\right)$. L'élément $\left(X_{\gamma}(0) \times \mathbb{G}_{m} \stackrel{p_{\gamma, \varepsilon}}{\rightarrow} X_{\infty} \times \mathbb{G}_{m}, \tau_{n}\right)$ appartient à $\operatorname{Var}_{X_{\infty} \times \mathbb{G}_{m}}^{\mathbb{G}_{m}}$. Par identification des actions via le morphisme fini de $\mathbb{G}_{m} \lambda \mapsto \lambda^{n} 1.1 .2$, la classe de cet objet dans $\mathcal{M}_{X_{\infty} \times \mathbb{G}_{m}}^{\mathbb{G}_{m}}$ ne dépend pas de $n$ :

$$
\left[X_{\gamma}(0) \times \mathbb{G}_{m} \stackrel{p_{\gamma, \varepsilon}}{\rightarrow} X_{\infty} \times \mathbb{G}_{m}, \tau_{n}\right]=\left[X_{\gamma}(0) \times \mathbb{G}_{m} \stackrel{p_{\gamma, \varepsilon}}{\rightarrow} X_{\infty} \times \mathbb{G}_{m}, \tau_{1}\right] .
$$

3.4.6. Preuve du théorème 3.3. - Nous donnons ici la trame de la preuve, les preuves des lemmes seront faîtes en 3.4.7.

Démonstration. - Par la proposition 3.8 remarquons tout d'abord que

Lemme 3.15. - Pour tout $\omega$ dans $\Omega$, l'arc $\left(P_{i}(t) / t^{\omega_{i}}\right)$ noté $\varphi$ appartient $\grave{a}$ $\mathscr{X}_{m_{\Gamma}(\omega), \omega}^{\delta}(f)$ si et seulement si le d-uplet $\left(P_{i}(0)\right)$ appartient $\grave{a} \mathbb{G}_{m}^{d} \backslash X_{\gamma(\omega)}(0)$. En ce cas, le coefficient angulaire ac $1 / f(\varphi)$ est égal à $\left.1 / f_{\gamma(\omega)} \underline{\left(P_{i}(0)\right.}\right)$.

Nous prouverons alors les deux lemmes de mesure suivant :

Lemme 3.16. - Soit $\gamma$ une face de $\Gamma, \varepsilon$ dans $\mathcal{E}, \omega$ dans $\sigma_{\gamma, \varepsilon}$, et $\delta$ un entier strictement positif, la mesure de $\mathscr{X}_{m_{\Gamma}(\omega), \omega}^{\delta}(f)$ vaut

$$
\mathbb{L}^{-\sum_{i=1}^{d}\left|\omega_{i}\right|}\left[\mathbb{G}_{m}^{d} \backslash X_{\gamma}(0), p_{\gamma, \varepsilon}, \sigma_{\gamma}\right] .
$$


Lemme 3.17. - Soit $\gamma$ une face de $\Gamma, \varepsilon$ dans $\mathcal{E}, \omega$ dans $\sigma_{\gamma, \varepsilon}, \delta$ un entier strictement positif, et $n$ dans $\left\{1, \ldots, m_{\Gamma}(\omega)-1\right\}$. Si $\mathscr{X}_{n, \omega}^{\delta}(f)$ est vide alors sa mesure est nulle sinon elle vaut $\left[X_{\gamma}(0) \times \mathbb{G}_{m} \stackrel{p_{\gamma, \varepsilon}}{\rightarrow} X_{\infty} \times \mathbb{G}_{m}, \tau_{1}\right] \mathbb{L}^{-p-\sum_{i=1}^{d}\left|w_{i}\right|}$ où $p$ est l'entier $m_{\Gamma}(\omega)-n$

Dans l'anneau $\mathcal{M}_{X_{\infty} \times \mathbb{G}_{m}}^{\mathbb{G}_{m}}[[T]]$, nous obtenons alors la décomposition suivante

$$
\begin{aligned}
Z_{1 / \hat{f}, U}^{\delta}(T)= & \sum_{\gamma \in \Gamma} \sum_{\varepsilon \in \mathcal{E}}\left[\mathbb{G}_{m}^{d} \backslash X_{\gamma}(0), p_{\gamma, \varepsilon}, \sigma_{\gamma}\right] S_{\gamma, \varepsilon}^{\delta,=}(\mathbb{L}, T) \\
& +\sum_{\gamma \in \Gamma} \sum_{\varepsilon \in \mathcal{E}}\left[X_{\gamma}(0) \times \mathbb{G}_{m}, p_{\gamma, \varepsilon}, \tau_{1}\right] S_{\gamma, \varepsilon}^{\delta,<}(\mathbb{L}, T)
\end{aligned}
$$

où il est noté avec les notations 3.4.3

$$
\begin{aligned}
S_{\gamma, \varepsilon}^{\delta,=}(\mathbb{L}, T) & :=\sum_{\omega \in C_{\gamma, \varepsilon}^{=} \cap \mathbb{Z}^{d}} \mathbb{L}^{-\sum_{i=1}^{d}\left|w_{i}\right|} T^{m_{\Gamma}(\omega)} \\
\text { et } S_{\gamma, \varepsilon}^{\delta,<}(\mathbb{L}, T) & :=\sum_{(n, \omega) \in C_{\gamma, \varepsilon}^{\delta,<} \cap \mathbb{Z}^{d+1}} \mathbb{L}^{-\left(m_{\Gamma}(\omega)-n\right)-\sum_{i=1}^{d}\left|w_{i}\right|} T^{n}
\end{aligned}
$$

Grâce au lemme 1.2 nous obtenons la rationalité et la limite des sommes précédentes :

LEMME 3.18. - La somme $S_{\gamma, \varepsilon}^{\delta,=}(\mathbb{L}, T)$ est rationnelle et sa limite vaut $\chi_{c}\left(C_{\gamma, \varepsilon}^{\delta,=}\right)$.

LEMME 3.19. - La somme $S_{\gamma, \varepsilon}^{\delta,<}(\mathbb{L}, T)$ est rationnelle et sa limite vaut 0.

Par application de ces deux lemmes, par additivité de la caractéristique d'Euler à support compact et par image directe nous obtenons alors l'expression suivante :

$$
S_{f, \infty}=-\sum_{\gamma \in \Gamma} \chi_{c}\left(C_{\gamma}^{\delta,=}\right)\left[\mathbb{G}_{m}^{d} \backslash X_{\gamma}(0), f_{\gamma}^{-1}, \sigma_{\gamma}\right] .
$$

Le lemme suivant conclut la preuve du théorème 3.3 :

Lemme 3.20. - Pour toute face $\gamma$ de $\Gamma$, si $\gamma$ est contenue dans une face contenant 0 alors la caractéristique d'Euler $\chi_{c}\left(C_{\gamma}^{\delta,=}\right)$ est nulle sinon elle vaut $(-1)^{d-\operatorname{dim} \gamma}$. 


\subsubsection{Preuves}

\subsubsection{Preuve du lemme 3.16.}

Démonstration. - Soit $\varphi$ un arc de la forme $\left(P_{i}(t) / t^{\omega_{i}}\right)$ un arc de $\mathscr{X}_{m_{\Gamma}(\omega), \omega}^{\delta}(f)$. Rappelons que $P_{i}(t)$ est une série formelle avec $P_{i}(0)$ non nul. Cet arc correspond à l'arc $\left(\left[x_{i, 0}(t): x_{i, 1}(t)\right],\left[f\left(P_{i}(t) / t^{\omega_{i}}\right)^{-1}: 1\right]\right)$ avec $\left(x_{i, 0}(t), x_{i, 1}(t)\right)$ de la forme $\left(t^{\omega_{i}} P_{i}(t)^{-1}, 1\right)$ pour $\omega_{i}>0,\left(1, P_{i}(t) t^{-\omega_{i}}\right)$ pour $\omega_{i}<0$ et $\left(1, P_{i}(t)\right)$ pour $\omega_{i}$ nul. On se place dans une carte affine contenant l'origine de l'arc et nous considérons $\left(t^{\left|\omega_{i}\right|} Q_{i}(t)\right)$ l'arc associé, où $Q_{i}(t)$ est $P_{i}(t)^{-\varepsilon_{i}}$, pour tout $i$ dans $\{1, \ldots, d\}$. On pose alors

$$
\mathscr{A}_{m_{\Gamma}(\omega), \omega}=\left\{t^{\left|\omega_{i}\right|} Q_{i}(t) \mid Q_{i}(t) \in k[[t]],\left(P_{i}(0)\right) \in \mathbb{G}_{m}^{d} \backslash X_{\gamma}(0)\right\}
$$

muni du morphisme convenable vers $X_{\infty} \times \mathbb{G}_{m}$ et de l'action standard du groupe $\mathbb{G}_{m}$ sur les arcs. Pour tout entier $k, \pi_{k}\left(\mathscr{A}_{m_{\Gamma}(\omega), \omega}\right)$ est isomorphe à $\pi_{k}\left(\mathscr{X}_{m_{\Gamma}(\omega), \omega}^{\delta}(f)\right)$ dans la catégorie $\operatorname{Var}_{X_{\infty} \times \mathbb{G}_{m}}^{\mathbb{G}_{m}}$. Dans la suite on tronque les arcs à l'ordre $n^{\prime}+k$ avec $n^{\prime}$ égal à $\sum_{i=1}^{d}\left|\omega_{i}\right|$ et $k$ dans $\mathbb{N}$. Par conséquent, $Q_{i}$ est tronqué à l'ordre $n^{\prime}+k-\left|\omega_{i}\right|$. Par le théorème [10,7.1] (que l'espace sous jacent soit lisse ou non) on obtient l'égalité

$$
\mu\left(\mathscr{X}_{m_{\Gamma}(\omega), \omega}^{\delta}(f)\right)=\mu\left(\mathscr{A}_{m_{\Gamma}(\omega), \omega}\right)=\lim _{k \rightarrow \infty}\left[\pi_{n^{\prime}+k}\left(\mathscr{A}_{m_{\Gamma}(\omega), \omega}\right)\right] . \mathbb{L}^{-\left(n^{\prime}+k\right) d}
$$

avec $\mathbb{L}$ la classe $\left[\mathbb{A}_{k}^{1} \times X_{\infty} \times \mathbb{G}_{m} \rightarrow X_{\infty} \times \mathbb{G}_{m}, \tau_{1}\right]$ où la flèche est la projection sur $X_{\infty} \times \mathbb{G}_{m}$ et l'action $\tau_{1}$ de $\mathbb{G}_{m}$ est $\tau_{1}(\lambda,(\mu, x, \nu))$ égal à $(\mu, x, \lambda \nu)$, pour tout $\lambda$ dans $\mathbb{G}_{m}$ et pour tout $(\mu, x, \nu)$ dans $\mathbb{A}_{k}^{1} \times X_{\infty} \times \mathbb{G}_{m}$. L'objet $\pi_{n^{\prime}+k}\left(\mathscr{A}_{m_{\Gamma}(\omega), \omega}\right)$ est une sous variété affine de $\mathbb{A}^{\left(n^{\prime}+k+1\right) d-\sum_{i=1}^{d}\left|w_{i}\right|}$ dont les coordonnées sont les coefficients des $Q_{i}$, notés $a_{i, j}$, avec $i$ appartenant à $\{1, \ldots, d\}$ et $j$ à $\left\{0, \ldots, n^{\prime}+\right.$ $\left.k-\left|\omega_{i}\right|\right\}$. On notera $a$ au lieu de $\left(a_{i, j}\right)$. L'action usuelle de $\mathbb{G}_{m}$ sur les arcs se traduit alors en coordonnées par $\sigma\left(\lambda,\left(a_{i, j}\right)\right)$ égal à $\left(\lambda^{j+\left|\omega_{i}\right|} a_{i, j}\right)$. Par conséquent,

$$
\begin{aligned}
F:\left(\pi_{n^{\prime}+k}\left(\mathscr{A}_{m_{\Gamma}(\omega), \omega}\right), \sigma\right) & \rightarrow\left(\mathbb{G}_{m}^{d} \backslash X_{\gamma}(0), \sigma_{\omega}\right) \\
\left(t^{\left|\omega_{i}\right|} Q_{i}(t)\right) & \mapsto \quad\left(P_{i}(0)\right)
\end{aligned}
$$

est un morphisme dans $\operatorname{Var}_{X}^{\mathbb{G}_{m}} \times \mathbb{G}_{m}$ compatible avec les flèches au dessus de $X_{\infty} \times \mathbb{G}_{m}$ et avec les actions $\sigma$ et $\sigma_{\omega}: F(\sigma(\lambda, a)), \sigma_{\omega}(\lambda, F(a))$ et $\left(\lambda^{-\omega_{i}} a_{i, 0}^{-\varepsilon_{i}}\right)$ sont égaux. Dans la catégorie $\operatorname{Var}_{X_{\infty} \times \mathbb{G}_{m}}^{\mathbb{G}_{m}}, F$ est une fibration triviale de fibre $\left(\mathbb{A}^{\left(n^{\prime}+k\right) d-\sum_{i=1}^{d}\left|w_{i}\right|} \times X_{\infty} \times \mathbb{G}_{m}, p_{X_{\infty} \times \mathbb{G}_{m}}, \sigma_{\omega}\right)$ avec $\sigma_{\omega}(\lambda,(a, x, \mu))$ égal à $\left(\left(\lambda^{j+\left|\omega_{i}\right|} a_{i, j}\right), x, \lambda^{m_{\Gamma}(\omega)} \mu\right)$ et $p_{X_{\infty} \times \mathbb{G}_{m}}$ la projection sur $X_{\infty} \times \mathbb{G}_{m}$.

Le morphisme de trivialisation $\Phi$ de $\left(\pi_{n^{\prime}+k}\left(\mathscr{A}_{m_{\Gamma}(\omega), \omega}\right), \sigma\right)$ vers le produit fibré

$$
\left(\mathbb{A}^{\left(n^{\prime}+k\right) d-\sum_{i=1}^{d}\left|w_{i}\right|} \times X_{\infty} \times \mathbb{G}_{m}, p_{X_{\infty} \times \mathbb{G}_{m}}, \sigma_{\omega}\right) \underset{X_{\infty} \times \mathbb{G}_{m}}{\times}\left(\mathbb{G}_{m}^{d} \backslash X_{\gamma}(0) \stackrel{p_{\gamma, \varepsilon}}{\rightarrow} X_{\infty} \times \mathbb{G}_{m}, \sigma_{\omega}\right)
$$


associe à $a$ le quadruplet $\left(a, \pi_{0}(a), f_{\gamma}\left(\left(a_{i, 0}^{-\varepsilon_{i}}\right)\right)^{-1},\left(a_{i, 0}^{-\varepsilon_{i}}\right)\right)$ où $\pi_{0}$ est le morphisme de $\left(\mathscr{A}_{m_{\Gamma}(\omega), \omega}, \sigma\right)$ vers $X_{\infty}$. Ce morphisme est un isomorphisme dans la catégorie $\operatorname{Var}_{X \infty}^{\mathbb{G}_{m} \times \mathbb{G}_{m}}$ d'inverse,

$$
\Phi^{-1}\left(a, \underline{x}, f_{\gamma}(\underline{x})^{-1}, \underline{x}\right)=a
$$

avec pour tout $i, a_{i, 0}^{-\varepsilon_{i}}$ égal à $x_{i}$. Par conséquent, dans l'anneau de Grothendieck $\mathcal{M}_{X_{\infty \times \mathbb{G} m}}^{\mathbb{G} m}$ on obtient l'égalité

$$
\left[\pi_{n^{\prime}+k}\left(\mathscr{A}_{m_{\Gamma}(\omega), \omega}\right), \sigma\right]=\left[\mathbb{A}^{\left(n^{\prime}+k\right) d-\sum_{i=1}^{d}\left|w_{i}\right|} \times X_{\infty} \times \mathbb{G}_{m}, \sigma_{\omega}\right]\left[\mathbb{G}_{m}^{d} \backslash X_{\gamma}(0), \sigma_{\omega}\right] .
$$

Par construction du groupe de Grothendieck $\mathcal{M}_{X_{\infty} \times \mathbb{G}_{m}}^{\mathbb{G}_{m}}$ on dispose de l'égalité

$$
\mathbb{L}^{\left(n^{\prime}+k\right) d-\sum_{i=1}^{d}\left|w_{i}\right|}=\left[\mathbb{A}^{\left(n^{\prime}+k\right) d-\sum_{i=1}^{d}\left|w_{i}\right|} \times X_{\infty} \times \mathbb{G}_{m}, \sigma_{\omega}\right] .
$$

En effet l'action $\sigma_{\omega}$ et l'action $\left.\tau_{m_{\Gamma}(\omega)}:\left(\lambda,\left(a_{j}^{i}\right), x, \mu\right)\right) \rightarrow\left(\left(a_{j}^{i}\right), x, \lambda^{m_{\Gamma}(\omega)} \mu\right)$ relèvent la même action sur $X_{\infty} \times \mathbb{G}_{m}$ donc induisent la même classe 1.1.2. Enfin $\mathbb{A}^{\left(n^{\prime}+k\right) d-\sum_{i=1}^{d}\left|w_{i}\right|} \times X_{\infty} \times \mathbb{G}_{m}$ munie de l'action $\tau_{m_{\Gamma(\omega)}}$ a la même classe que la même variété munie de l'action $\tau_{1}$ car on passe d'une action à l'autre par le morphisme fini de $\mathbb{G}_{m}, \lambda \mapsto \lambda^{m_{\Gamma}(\omega)}$ 1.1.2. Ainsi dans $\mathcal{M}_{X_{\infty} \times \mathbb{G}_{m}}^{\mathbb{G}_{m}}$ la classe $\left[\pi_{n^{\prime}+k}\left(\mathscr{A}_{m_{\Gamma}(\omega), \omega}\right), \sigma\right]$ est égale à $\mathbb{L}^{\left(n^{\prime}+k\right) d-\sum_{i=1}^{d}\left|w_{i}\right|}\left[\mathbb{G}_{m}^{d} \backslash X_{\gamma}(0), p_{\gamma, \varepsilon}, \sigma_{\gamma}\right]$ ce qui prouve le lemme 3.16.

\subsubsection{Preuve du lemme 3.1\%.}

Démonstration. - Soit $\varphi$ un arc de $\mathscr{X}_{n, \omega}^{\delta}(f)$ égal à $\left(P_{i}(t) / t^{\omega_{i}}\right)$. Rappelons que $P_{i}(t)$ est une série formelle avec $P_{i}$ non nul. Cet arc correspond à l'arc tracé sur $X$ noté $\left(\left[x_{i, 0}(t): x_{i, 1}(t)\right],\left[f\left(P_{i}(t) / t^{\omega_{i}}\right)^{-1}: 1\right]\right)$ avec $\left(x_{i, 0}(t), x_{i, 1}(t)\right)$ de la forme $\left(t^{\omega_{i}} P_{i}(t)^{-1}, 1\right)$ pour $\omega_{i}>0,\left(1, P_{i}(t) t^{-\omega_{i}}\right)$ pour $\omega_{i}<0$ et $\left(1, P_{i}(t)\right)$ pour $\omega_{i}$ nul. On se place dans une carte affine contenant l'origine de l'arc et nous considérons $\left(t^{\left|\omega_{i}\right|} Q_{i}(t)\right)$ l'arc associé, où $Q_{i}(t)$ est $P_{i}(t)^{-\varepsilon_{i}}$, pour tout $i$ dans $\{1, \ldots, d\}$ et avec les notations précédentes on pose alors

$$
\mathscr{A}_{n, \omega}=\left\{\begin{array}{l|l}
t^{\left|\omega_{i}\right|} Q_{i}(t) & \begin{array}{l}
Q_{i}(t) \in k[[t]],\left(P_{i}(0)\right) \in X_{\gamma}(0) \\
\text { et } \operatorname{ord}_{t} f\left(P_{i}(t) / t^{\omega_{i}}\right)^{-1}=n
\end{array}
\end{array}\right\}
$$

avec pour convention signe $(0)$ égal à -1 . On le munit du morphisme convenable vers $X_{\infty} \times \mathbb{G}_{m}$ et de l'action standard du groupe $\mathbb{G}_{m}$ sur les arcs. Pour tout entier $k, \pi_{k}\left(\mathscr{A}_{n, \omega}\right)$ est isomorphe à $\pi_{k}\left(\mathscr{X}_{n, \omega}^{\delta}(f)\right)$ dans la catégorie $\operatorname{Var}_{X_{\infty} \times \mathbb{G}_{m}}^{\mathbb{G}_{m}}$. On note $n^{\prime}$ l'entier $n+\sum_{i=1}^{d}\left|w_{i}\right|$, on tronque les arcs au rang $n^{\prime}+k$ avec $k$ dans $\mathbb{N}$ et on dispose des égalités

$$
\mu\left(\mathscr{X}_{n, \omega}^{\delta}(f)\right)=\mu\left(\mathscr{A}_{n, \omega}(f)\right)=\lim _{k \rightarrow \infty}\left[\pi_{n^{\prime}+k}\left(\mathscr{A}_{n, \omega}(f)\right)\right] \mathbb{L}^{-\left(n^{\prime}+k\right) d} \in \mathcal{M}_{X_{\infty} \times \mathbb{G}_{m}}^{\mathbb{G}_{m}} .
$$


Pour tout $i$ dans $\{1, \ldots, d\}$, l'arc $Q_{i}$ est tronqué à l'ordre $n^{\prime}+k-\left|\omega_{i}\right|$. L'objet $\pi_{n^{\prime}+k}\left(\mathscr{A}_{n, \omega}\right)$ est une sous variété affine de $\mathbb{A}^{\left(n^{\prime}+k+1\right) d-\sum_{i=1}^{d}\left|w_{i}\right|}$ dont les coordonnées sont les coefficients des $Q_{i}$, notés $a_{i, j}$, avec $i$ dans $\{1, \ldots, d\}$ et $j$ dans $\left\{0, \ldots, n^{\prime}+k-\left|\omega_{i}\right|\right\}$. L'action usuelle de $\mathbb{G}_{m}$ sur les arcs se traduit alors en coordonnées par $\sigma\left(\lambda,\left(a_{i, j}\right)\right)$ égal à $\left(\lambda^{j+\left|\omega_{i}\right|} a_{i, j}\right)$.

Par le lemme 3.15, pour tout $\left(t^{\left|\omega_{i}\right|} Q_{i}(t)\right)$ appartenant à $\pi_{n^{\prime}+k}\left(\mathscr{A}_{n, \omega}(f)\right)$, $\left(P_{i}(0)\right)$ appartient à $X_{\gamma}(0)$. Par conséquent, dans la catégorie $\operatorname{Var}_{X_{\infty} \times \mathbb{G}_{m}}^{\mathbb{G}_{m}}$, le morphisme

$$
\begin{aligned}
F: \pi_{n^{\prime}+k}\left(\mathscr{A}_{n, \omega}(f)\right) & \rightarrow \quad X_{\gamma}(0) \times \mathbb{G}_{m} \\
t^{\left|\omega_{i}\right|} Q_{i}(t) & \mapsto\left(\left(P_{i}(0)\right), a c\left(f\left(P_{i}(t) / t^{\omega_{i}}\right)^{-1}\right)\right)
\end{aligned}
$$

est compatible avec les flèches et les actions $\sigma$ et $\tau_{n}$. Comme $f$ est non dégénéré pour son polyèdre de Newton à l'infini, $f_{\gamma}$ est lisse sur $\mathbb{G}_{m}^{d}$. Donc pour tout $a$ dans $X_{\gamma}(0)$ il existe $i_{0}$ dans $\{1, \ldots, d\}$ tel que $\frac{\partial f_{\gamma}}{\partial x_{i_{0}}}(a)$ est non nul, donc $\frac{\partial \tilde{f}_{\gamma}}{\partial x_{i_{0}}}(a, 0)$ est non nul. Montrons que dans la catégorie $\operatorname{Var}_{X_{\infty} \times \mathbb{G}_{m}}^{\mathbb{G}_{m}}, F$ est une fibration localement triviale de fibre

$$
\left(\mathbb{A}^{\left(n^{\prime}+k\right) d-\sum_{i=1}^{d}\left|\omega_{i}\right|-p} \times X_{\infty} \times \mathbb{G}_{m}, p r_{X_{\infty} \times \mathbb{G}_{m}}, \sigma\right)
$$

avec comme ouverts de trivialisation les variétés $\left(X_{\gamma}(0) \cap\left(\frac{\partial f_{\gamma}}{\partial x_{i_{0}}} \neq 0\right)\right) \times$ $\mathbb{G}_{m}$ et comme morphismes de trivialisation, les morphismes $\Phi$ de $\{\varphi \in$ $\left.\pi_{n^{\prime}+k}\left(\mathscr{A}_{n, \omega}(f)\right) \mid \frac{\partial f_{\gamma}}{\partial x_{i_{0}}}\left(\left(P_{i}(0)\right)\right) \neq 0\right\}$ que nous notons

$$
\left(\pi_{n^{\prime}+k}\left(\mathscr{A}_{n, \omega}\right) \cap \frac{\partial f_{\gamma}}{\partial x_{i_{0}}} \neq 0, \sigma\right)
$$

vers le produit fibré

$\left(\mathbb{A}^{\left(n^{\prime}+k\right) d-\sum_{i=1}^{d}\left|\omega_{i}\right|-p} \times X_{\infty} \times \mathbb{G}_{m}, p r_{X_{\infty} \times \mathbb{G}_{m}}, \sigma\right) \underset{X_{\infty} \times \mathbb{G}_{m}}{\times}\left(X_{\gamma}(0) \times \mathbb{G}_{m}, p_{\gamma, \varepsilon}, \tau_{n}\right)$.

Il associe à chaque arc tronqué $\left(t^{\left|\omega_{i}\right|} Q_{i}(t)\right)$, écrit en coordonnées $\left(a_{i, j}\right)$, le quadruplet

$$
\left(a_{i, 0}^{-\varepsilon_{i}}, \text { ac } f\left(P_{i}(t) / t^{\omega_{i}}\right)^{-1} ;\left(a_{i, j}\right)_{(i, j) \in I_{i_{0}}}, \pi_{0}\left(a_{i, j}\right), \text { ac } f\left(P_{i}(t) / t^{\omega_{i}}\right)^{-1}\right)
$$

où $\pi_{0}$ est le morphisme de $\left(\pi_{n^{\prime}+k}\left(\mathscr{A}_{n, \omega}\right), \sigma\right)$ vers $X_{\infty}$ et $I_{i_{0}}$ est l'ensemble d'indices

$I_{i_{0}}:=\left\{(i, j) \mid i \neq i_{0}, j \in\left\{1 \ldots n^{\prime}+k-\left|w_{i}\right|\right\}\right\} \cup\left\{\left(i_{0}, j\right) \mid j \in\left\{p+1, \ldots, n^{\prime}+k-\left|w_{i_{0}}\right|\right\}\right\}$.

Montrons que $\Phi$ est un isomorphisme : soit $\left(t^{\left|\omega_{i}\right|} Q_{i}(t)\right)$ dans $\pi_{n^{\prime}+k}\left(\mathscr{A}_{n, \omega}(f)\right)$. Il existe $A$ une série formelle inversible telle que

$$
\frac{1}{f(\varphi(t))}=\frac{t^{m_{\Gamma}(\omega)}}{\tilde{f}_{\gamma}\left(\left(P_{i}(t)\right), t\right)}=A(t) t^{n}, A(0) \neq 0
$$

TOME $140-2012-\mathrm{N}^{\mathrm{O}} 1$ 
On note $p$ l'entier $m_{\Gamma}(a)-n$. En ce cas $\tilde{f}_{\gamma}\left(\left(P_{i}(t)\right), t\right)$ vaut $A^{-1}(t) t^{p}$ et $\tilde{f}_{\gamma}\left(\left(P_{i}(t)\right), t\right)$ est égal à $t^{p} / A(0) \bmod t^{p+1}$. Rappelons que

$$
\tilde{f}_{\gamma}(\underline{x}, u)=f_{\gamma(\omega)}(\underline{x})+\sum_{k \notin \gamma} \alpha_{\underline{k}} u^{m_{\Gamma}(\underline{\omega})-\underline{k} \cdot \underline{\omega}} \underline{x}^{\underline{k}} .
$$

Par le développement de Taylor on peut écrire :

$\tilde{f}_{\gamma}(P(t), t)=\tilde{f}_{\gamma}(P(0), 0)+D \tilde{f}_{\gamma}(P(0), 0)(P(t)-P(0), t)+\cdots=\frac{1}{A(0)} t^{p} \bmod t^{p+1}$.

Or par hypothèse $\tilde{f}_{\gamma}(P(0), 0)$ et $f_{\gamma}(P(0))$ sont nuls, donc en notant $\left(x_{n+1}, P_{n+1}(t)\right)$ le couple de variables $(u, t)$, on dispose l'égalité

$$
\sum_{k=1}^{p} \frac{1}{k !} \sum_{i_{1}, \ldots, i_{k}} \frac{\partial^{k} \tilde{f}_{\gamma}}{\partial x_{i_{1}} \cdots \partial x_{i_{k}}}(P(0), 0) \prod_{j=1}^{k}\left(P_{i_{j}}(t)-P_{i_{j}}(0)\right)=\frac{1}{A(0)} t^{p} \bmod t^{p+1}
$$

Pour tout $i$ dans $\{1, \ldots, n\}, \frac{\partial \tilde{f}_{\gamma}}{\partial x_{i}}(P(0), 0)$ est égal à $\frac{\partial f_{\gamma}}{\partial x_{i}}(P(0))$. En isolant la variable $i_{0}$ nous obtenons l'égalité

$$
\begin{aligned}
\frac{\partial \tilde{f}_{\gamma}}{\partial x_{i_{0}}}( & P(0), 0)\left(P_{i_{0}}(t)-P_{i_{0}}(0)\right) \\
& +\sum_{k=2}^{p} \sum_{i_{1}, \ldots, i_{k-1}} \frac{1}{k !} \frac{\partial^{k} \tilde{f}_{\gamma}}{\partial x_{i_{0}} \cdots \partial x_{i_{k-1}}}(P(0), 0) \prod_{j=0}^{k-1}\left(P_{i_{j}}(t)-P_{i_{j}}(0)\right) \\
= & \frac{1}{\alpha(0)} t^{p}-\sum_{\substack { k=1 \\
\begin{subarray}{c}{i_{1} \neq i_{0} \\
\ldots \neq \\
i_{k} \neq i_{0}{ k = 1 \\
\begin{subarray} { c } { i _ { 1 } \neq i _ { 0 } \\
\ldots \neq \\
i _ { k } \neq i _ { 0 } } }\end{subarray}} \frac{1}{k !} \frac{\partial^{k} \tilde{f}_{\gamma}}{\partial x_{i_{1}} \cdots \partial x_{i_{k}}}(P(0), 0) \prod_{j=1}^{k}\left(P_{i_{j}}(t)-P_{i_{j}}(0)\right) \bmod t^{p+1},
\end{aligned}
$$

ainsi que l'inégalité sur les ordres

$$
\begin{aligned}
& \operatorname{ord}_{t} \frac{\partial \tilde{f}_{\gamma}}{\partial x_{i_{0}}}(P(0), 0)\left(P_{i_{0}}(t)-P_{i_{0}}(0)\right) \\
& \quad<\operatorname{ord}_{t}\left(\sum_{k=2}^{p} \sum_{i_{1}, \ldots, i_{k-1}} \frac{1}{k !} \frac{\partial^{k} \tilde{f}_{\gamma}}{\partial x_{i_{0}} \cdots \partial x_{i_{k-1}}}(P(0), 0) \prod_{j=0}^{k-1}\left(P_{i_{j}}(t)-P_{i_{j}}(0)\right)\right)
\end{aligned}
$$

Donc les $p$ premiers coefficients de $P_{i_{0}}: a_{1}^{i_{0}}, \ldots, a_{p}^{i_{0}}$ s'obtiennent de manière unique à partir des $\left(a_{i, j}\right)_{(i, j) \in I_{i_{0}}}$ comme solutions d'un système triangulaire dont la solution est unique car $\frac{\partial f_{\gamma}}{\partial x_{i_{0}}}(P(0), 0) \neq 0$. Par conséquent, on obtient 
dans l'anneau $\mathcal{M}_{X_{\infty} \times \mathbb{G}_{m}}^{\mathbb{G}_{m}}$ le produit

$$
\begin{aligned}
& {\left[\pi_{n^{\prime}+k}\left(\mathscr{A}_{n, \omega}(f)\right) \cap \frac{\partial f_{\gamma}}{\partial x_{i_{0}}} \neq 0, \sigma\right]} \\
& \quad=\left[X_{\gamma}(0) \times \mathbb{G}_{m}, p_{\gamma, \varepsilon}, \tau_{n}\right]\left[\mathbb{A}^{\left(n^{\prime}+k\right) d-\sum_{i=1}^{d}\left|\omega_{i}\right|-p} \times X_{\infty} \times \mathbb{G}_{m}, \sigma_{\omega}\right] .
\end{aligned}
$$

Comme précédemment par identification des actions nous avons les égalités

$$
\left[\mathbb{A}^{\left(n^{\prime}+k\right) d-\sum_{i=1}^{d}\left|\omega_{i}\right|-p} \times X_{\infty} \times \mathbb{G}_{m}, \sigma_{\omega}\right]=\mathbb{L}^{\left(n^{\prime}+k\right) d-\sum_{i=1}^{d}\left|w_{i}\right|-p}
$$

et $\left[X_{\gamma}(0) \times \mathbb{G}_{m}, p_{\gamma, \varepsilon}, \tau_{n}\right]=\left[X_{\gamma}(0) \times \mathbb{G}_{m}, p_{\gamma, \varepsilon} \times \mathbb{G}_{m}, \tau_{1}\right]$. Ceci prouve le lemme 3.17 .

\subsubsection{Preuve du lemme 3.18}

Démonstration. - Fixons $\gamma$ et $\varepsilon$, le cône $C_{\gamma, \varepsilon}^{\delta,=}$ peut être vide, la somme $S_{\gamma, \varepsilon}^{\delta,=}(\mathbb{L}, T)$ est alors nulle. Ecartons ce cas là. La preuve découle des lemmes 1.2 et 3.21 .

Lemme 3.21. - Pour un polynôme de Laurent, pour un des cônes précédents $C_{\gamma, \varepsilon}$, on considère les formes linéaires $l: \omega \mapsto(a \mid \omega)$ avec a dans $\gamma$ et $\nu: \omega \mapsto$ $\sum_{i=1}^{d} \varepsilon_{i} w_{i}$. La forme linéaire l est strictement positive sur l'adhérence $\overline{C_{\gamma, \varepsilon}} \backslash\{0\}$ et $\nu$ est positive.

Démonstration. - Par définition de $\sigma_{\gamma, \varepsilon}$, pour tout $\omega$ appartenant au cône $C_{\gamma, \varepsilon}, \nu(\omega)$ vaut $\sum_{i=1}^{d}\left|w_{i}\right|$. Par continuité, cette forme linéaire ne s'annule qu'en 0 sur $\overline{C_{\gamma, \varepsilon}}$. Soit $\gamma$ une face du type $\left(\gamma_{l_{1}} \cap \cdots \cap \gamma_{l_{s}}\right) \cap\left(\gamma_{k_{1}} \cap \cdots \cap \gamma_{k_{t}}\right)$ où les $\gamma_{l_{i}}$ sont les faces de codimension 1 contenant 0 et les $\gamma_{k_{j}}$ sont les faces ne contenant pas 0 . Pour $\gamma_{i}$ une face de codimension 1 on note $\vec{\alpha}^{(i)}$ la normale à la face $\gamma_{i}$ extérieure au polyèdre $\Gamma$ et de plus petite norme. Par définition $3.8, \sigma(\gamma)$ est égal à $\sum_{i=1}^{s} \mathbb{R}_{+}^{*} \vec{\alpha}^{\left(l_{i}\right)}+\sum_{i=1}^{t} \mathbb{R}_{+}^{*} \vec{\alpha}^{\left(k_{i}\right)}$ et son adhérence $\overline{\sigma(\gamma)}$ est $\sum_{i=1}^{s} \mathbb{R}_{+} \vec{\alpha}^{\left(l_{i}\right)}+\sum_{i=1}^{t} \mathbb{R}_{+} \vec{\alpha}^{\left(k_{i}\right)}$. L'adhérence du cône $C_{\gamma, \varepsilon}$ est contenue dans $\overline{\sigma(\gamma)}$. Soit $\vec{a}$ dans $\gamma$, pour tout $i$ dans $\{1, \ldots, s\}$, le produit scalaire $\left(\vec{a} \mid \vec{\alpha}^{\left(l_{i}\right)}\right)$ est nul car $\vec{a}$ appartient à $\gamma_{l_{i}}$ qui contient 0 et la forme linéaire $\left(\vec{\alpha}^{\left(l_{i}\right)} \mid\right.$.) atteint son maximum sur la face $\gamma_{l_{i}}$, par 3.9. Par conséquent, tout $\omega$ dans $\sigma(\gamma)$ s'écrit sous la forme $\sum_{i=1}^{s} \lambda_{l_{i}} \vec{\alpha}^{\left(l_{i}\right)}+\sum_{i=1}^{t} \lambda_{k_{i}} \vec{\alpha}^{\left(k_{i}\right)}$, et le produit scalaire $(a \mid \omega)$ est égal à $\sum_{i=1}^{t} \lambda_{k_{i}}\left(\vec{a} \mid \vec{\alpha}^{\left(k_{i}\right)}\right)$. Or pour tout $i$ dans $\{1, \ldots, t\}$, le vecteur $\vec{\alpha}^{\left(k_{i}\right)}$ appartient à $\Omega$ donc le produit scalaire $\left(\vec{a} \mid \vec{\alpha}^{\left(k_{i}\right)}\right)$ vaut $m_{\Gamma}\left(\vec{\alpha}^{\left(k_{i}\right)}\right)$ et est donc strictement positif. Par conséquent $(a \mid \omega)$ est négatif ou nul, et en particulier nul si tous les $\lambda_{k_{i}}$ sont nuls, auquel cas $m_{\Gamma}(\omega)$ est lui même nul. Or pour tout $i$ dans $\{1, \ldots, d\}$ les inégalités $\delta m_{\Gamma}(\omega) \geq \omega_{i} \geq-\delta m_{\Gamma}(\omega)$ restent 
vraies sur l'adhérence du cône donc $\omega_{i}$ est nul, pour tout $i$ dans $\{1, \ldots, d\}$. Par conséquent la forme linéaire $l$ est strictement positive sur $\overline{C_{\gamma, \varepsilon}} \backslash\{0\}$.

Soit $\gamma$ une face du type $\gamma_{k_{1}} \cap \cdots \cap \gamma_{k_{t}}$ où les $\gamma_{k_{j}}$ sont des faces de codimension 1 ne contenant pas 0 . L'adhérence $\overline{\sigma(\gamma)}$ est égale à $\sum_{i=1}^{t} \mathbb{R}_{+} \vec{\alpha}^{\left(k_{i}\right)}$. L'adhérence $\overline{C_{\gamma, \varepsilon}}$ du cône $C_{\gamma, \varepsilon}$ est contenue dans $\overline{\sigma(\gamma)}$. Soit $\vec{a}$ un point de la face $\gamma$ et $\omega$ un élément de $\overline{\sigma(\gamma)}$ que l'on écrit sous la forme $\sum_{i=1}^{t} \lambda_{k_{i}} \vec{\alpha}^{\left(k_{i}\right)}$, en ce cas le produit scalaire $(a \mid \omega)$ vaut $\sum_{i=1}^{t} \lambda_{k_{i}}\left(\vec{a} \mid \vec{\alpha}^{\left(k_{i}\right)}\right)$. Pour tout $i$ dans $\{1, \ldots, t\}$, le vecteur $\vec{\alpha}^{\left(k_{i}\right)}$ appartient à $\Omega$, donc le produit scalaire $\left(\vec{a} \mid \vec{\alpha}^{\left(k_{i}\right)}\right)$ vaut $m_{\Gamma}\left(\vec{\alpha}^{\left(k_{i}\right)}\right)$ et est donc strictement positif. Par conséquent $(a \mid \omega)$ est positif ou nul et en particulier nul si tous les $\lambda_{k_{i}}$ sont nuls c'est à dire $\omega$ nul. La forme linéaire $l$ est strictement positive sur $\overline{C_{\gamma, \varepsilon}} \backslash\{0\}$.

\subsubsection{Preuve du lemme 3.19}

Démonstration. - Montrons tout d'abord que pour tout $\delta>0$, pour tout $\gamma, \varepsilon$ tels que $C_{\gamma, \varepsilon}^{\delta,<}$ soit non vide, le cône $C_{\gamma, \varepsilon}^{\delta,<}$ est polyédral rationnel convexe de caractéristique d'Euler nulle. Nous pouvons supposer que pour tout $i, \varepsilon_{i}$ est égal à 1 , autrement dit $\omega_{i}>0$. Notons $C$ le cône polyédral rationnel convexe relativement ouvert $\left\{n \in \mathbb{N}^{*}, \omega \in \sigma_{\gamma, \varepsilon} \mid \underline{m}_{\Gamma}(\omega)>n\right\}$. Le cône $C_{\gamma, \varepsilon}^{\delta,<}$ est alors égal à $\left\{(n, \omega) \in C \mid \forall i, n \delta \geq \omega_{i}\right\}$. Notons que toutes les inégalités ne sont pas toutes trivialement vérifiées, autrement dit $C_{\gamma, \varepsilon}^{\delta,<}$ est différent de $C$. Ce cône est la réunion disjointe de cônes polyédraux rationnels convexes (parfois vides) de la forme

$$
C_{I, \geq}:=\left\{\begin{array}{l|l}
n, \omega) \in C & \begin{array}{l}
\omega_{i}=\omega_{j} \\
n \delta \geq \omega_{i}>\omega_{k}
\end{array} \forall i \in I, \forall k \notin I
\end{array}\right\}
$$

(pour toute partie $I$ non vide de $\{1, \ldots, d\}$ ) où l'inégalité n'est pas triviale. Ce cône est lui même contenu dans le cône polyédral rationnel convexe $\left\{(n, \omega) \in C \mid \omega_{i}=\omega_{j}>\omega_{k} \forall i, j \in I, \forall k \notin I\right\}$ noté $C_{I}$ et son complémentaire est le cône $\left\{(n, \omega) \in C_{I} \mid n \delta<\omega_{i}, i \in I\right\}$ qui est un cône polyédral rationnel convexe relativement ouvert non vide et de même dimension que $C_{I}$. Ainsi par additivité de la caractéristique d'Euler à support compact, $\chi_{c}\left(C_{I, \geq}\right)$ est nulle. En particulier par sommation, $\chi_{c}\left(C_{\gamma, \varepsilon}^{\delta,<}\right)$ est nulle.

Considérons les formes linéaires $l:(n, \omega) \mapsto n$ et $\nu:(n, \omega) \mapsto\left(m_{\Gamma}(\omega)-n\right)+$ $\sum_{i=1}^{d}\left|\omega_{i}\right|$. Ces deux formes linéaires sont strictement positives sur $\overline{C_{\gamma, \varepsilon}^{\delta,<} \backslash\{0\}}$. Si $n$ est nul par la condition au bord $m_{\Gamma}(\omega)>n \geq\left|\omega_{i}\right| / \delta$ alors tous les $\omega_{i}$ sont nuls. Par conséquent par le lemme 1.2 la somme $S_{\gamma, \varepsilon}^{\delta,<}(\mathbb{L}, T)$ est rationnelle et sa limite vaut 0 . 


\subsubsection{Preuve du lemme 3.20}

Démonstration. - Rappelons que $C_{\gamma}^{\delta,=}$ est le cône $\left\{\omega \in \sigma(\gamma)|| \omega_{i} \mid \leq m_{\Gamma}(\omega) \delta\right\}$.

Soit $\gamma$ une face de $\Gamma$ non contenue dans une face contenant 0 . Montrons les inégalités $-m_{\Gamma}(\omega) \delta \leq \omega_{j} \leq m_{\Gamma}(\omega) \delta$ pour tout $\omega$ dans $\sigma(\gamma)$ et pour tout $j$ dans $\{1, \ldots, d\}$. Cette face est une intersection de faces $\gamma_{j}$ de codimension 1 qui ne contiennent pas 0 , par exemple $\gamma$ est égal à $\gamma_{1} \cap \cdots \cap \gamma_{t}$. Le cône $\sigma(\gamma)$ est alors égal à $\sum_{j=1}^{t} \mathbb{R}_{*}^{+} \alpha^{(j)}$ où $\alpha^{(j)}$ est le vecteur normal de la face $\gamma_{j}$, extérieur à $\Gamma$, à coordonnées entières et de plus petite norme. Soit $\omega$ appartenant à $\sigma(\gamma)$, il existe $\left(\lambda_{j}\right)$ dans $\mathbb{R}_{*}^{+}$tels que $\omega$ s'écrivent $\sum_{j=1}^{t} \lambda_{j} \alpha^{(j)}$. Pour toute face $\gamma_{j}$, pour tout $a$ de la face $\gamma_{j},\left(a \mid \alpha^{(j)}\right)$ vaut $m_{\Gamma}\left(\alpha^{(j)}\right)$ et est donc strictement positif car $\gamma_{j}$ ne contient pas 0 . Par la proposition 3.8, $m_{\Gamma}(\omega)$ est égal à $(a \mid \omega)$ soit à $\sum_{j=1}^{t} \lambda_{j} m_{\Gamma}\left(\alpha^{(j)}\right)>0$ pour un élément $a$ de la face $\gamma$. Si pour tout $i, j, \delta$ est inférieur à $\left|\alpha_{i}^{(j)}\right| / m_{\Gamma}\left(\alpha^{(j)}\right)$, alors $\left|\omega_{i}\right|$ est supérieur à $m_{\Gamma}(\omega) \delta$. Ceci donne un nombre fini de conditions sur $\delta$ qui ne dépendent que de $\Gamma$ et sont vérifiées pour $\delta$ assez grand. Ainsi, pour $\delta$ suffisament grand le cône $C_{\gamma}^{\delta,=}$ est égal à $\sigma(\gamma)$ qui est un cône rationnel relativement ouvert de $\operatorname{dimension} d-\operatorname{dim} \gamma$ et de caractéristique d'Euler $(-1)^{d-\operatorname{dim} \gamma}$.

Soit $\gamma$ une face contenue dans une face contenant 0 , on peut par exemple écrire $\gamma$ sous la forme $\gamma_{1} \cap \cdots \cap \gamma_{l} \cap \gamma_{l+1} \cap \cdots \cap \gamma_{t}$ où les $\gamma_{i}$ sont des faces de codimension 1 et pour $i$ dans $\{1, \ldots, l\}, 0$ appartient à la face $\gamma_{i}$. Le cône $\sigma(\gamma)$ est égal à $\sum_{j=1}^{t} \mathbb{R}_{*}^{+} \alpha^{(j)}$ où $\alpha^{(j)}$ est le vecteur normal de la face $\gamma_{j}$, extérieur à $\Gamma$, à coordonnées entières et de plus petite norme. Soit $\omega$ un élément du cône $\sigma(\gamma)$ il existe $\left(\lambda_{i}\right)$ dans $\left(\mathbb{R}_{+}^{*}\right)^{l}$ et $\left(\mu_{j}\right)$ dans $\left(\mathbb{R}_{+}^{*}\right)^{t-l}$ tels que $\omega$ s'écrive sous la forme $\sum_{j=1}^{l} \lambda_{j} \alpha^{(j)}+\sum_{j=l+1}^{t} \mu_{j} \alpha^{(j)}$. Pour $a$ appartenant à $\gamma, m_{\Gamma}(\omega)$ vaut $\sum_{j=l+1}^{t} \mu_{j}\left(\alpha^{(j)} \mid a\right)$. En particulier comme $\sum_{j=1}^{l} \lambda_{j} \alpha^{(j)}$ n'est pas nul (sinon $\omega$ appartiendrait au cône $\left.\sigma\left(\cap_{i=l+1}^{t} \gamma_{t}\right)\right)$ toutes les conditions $-m_{\Gamma}(\omega) \delta \leq \omega_{i} \leq$ $m_{\Gamma}(\omega) \delta$ ne sont pas trivialement vérifiées pour tout $\omega$ dans $C_{\gamma}^{\delta,=}$ et $i$ dans $\{1, \ldots, d\}$. Montrons alors que le cône $C^{\delta,=}$ est de caractéristique d'Euler nulle. Commençons par le stratifier en sous cône $C_{\gamma, \varepsilon}^{\delta,=}$ avec $\varepsilon$ dans $\mathcal{E}$. Et montrons que pour tout $\varepsilon$ le cône $C_{\gamma, \varepsilon}^{\delta,=}$ est de caractéristique d'Euler nulle. On peut supposer $\omega_{i}>0$ pour tout $i$ et comme en 3.19 on stratifie $C_{\gamma, \varepsilon}^{\delta,=}$ en sous-cônes polyédraux rationnels convexes (parfois vides) :

$$
C_{I, \geq}:=\left\{\begin{array}{ll}
\omega \in \sigma(\gamma, \varepsilon) & \begin{array}{l}
\omega_{i}=\omega_{j} \\
m_{\Gamma}(\omega) \delta \geq \omega_{i}>\omega_{k}
\end{array} \forall i \in I, \forall k \notin I
\end{array}\right\}
$$

(pour toute partie $I$ non vide de $\{1, \ldots, d\}$ ) où l'inégalité n'est pas triviale. Ce cône est lui même contenu dans le cône polyédral rationnel convexe $\left\{\omega \in \sigma(\gamma, \varepsilon) \mid \omega_{i}=\omega_{j}>\omega_{k} \forall i, j \in I, \forall k \notin I\right\}$ noté $C_{I}$ et son complémentaire est le cône $\left\{\omega \in C_{I} \mid m_{\Gamma}(\omega) \delta<\omega_{i}, i \in I\right\}$ qui est un cône polyédral rationnel 
convexe non vide relativement ouvert et de même dimension que $C_{I}$. Ainsi par additivité de la caractéristique d'Euler à support compact, $\chi_{c}\left(C_{I, \geq}\right)$ est nulle. En particulier par sommation $\chi_{c}\left(C_{\gamma, \varepsilon}^{\delta,<}\right)$ puis $\chi_{c}\left(C_{\gamma}^{\delta,<}\right)$ sont nulles.

3.5. Calcul de la fibre de Milnor motivique à l'infini et du spectre à l'infini. - Soit $f$ un polynôme de Laurent appartenant à $k\left[x_{1}, \ldots, x_{d}\right]\left[x_{1}^{-1}, \ldots, x_{d}^{-1}\right]$. Son domaine de définition est noté $\mathcal{U}$. Dans cette partie nous calculons la fibre de Milnor motivique à l'infini de $f: \mathcal{U} \rightarrow \mathbb{G}_{m}$. Nous utilisons le résultat précédent 3.3 et l'additivité de la fibre de Milnor motivique.

Dans l'espace affine $\mathbb{A}_{k}^{d}$ égal à Spec $k\left[x_{1}, \ldots, x_{d}\right]$, on note $E_{i}$ l'hyperplan d'équation $x_{i}=0$ et on désignera par $E$ le diviseur $\sum_{i=1}^{d} E_{i}$. L'espace $\mathbb{A}_{k}^{d}$ est alors union disjointe des $E_{I}^{0}$ où pour tout partie $I$ de $\{1, \ldots, d\}, E_{I}^{0}$ est le constructilble $\cap_{i \in I} E_{i} \backslash \cup_{j \notin I} E_{j}$ et $E_{\varnothing}^{0}$ est le complémentaire du diviseur $\mathbb{A}_{k}^{d} \backslash E$.

Notons $\mathscr{P}(\{1, \ldots, d\})$ l'ensemble des parties de $\{1, \ldots, d\}$. Il existe une partie $I$ de $\mathscr{P}(\{1, \ldots, d\})$ telle que $U$ soit égal à l'union disjointe des $E_{I}^{0}$ où $I$ appartient à $\mathcal{I}$.

Ceci stratifie $i\left(\mathcal{U} \backslash f^{-1}(0)\right)$ en la réunion disjointe $\bigsqcup_{I \in \mathcal{J}} i\left(E_{I}^{0} \backslash f^{-1}(0)\right)$. Rappelons que nous travaillons avec la compactification $(X, i, \hat{f})$ définie en 3.2.

Par additivité du morphisme $S_{1 / \hat{f}}: M_{X \backslash X_{0}} \rightarrow M_{X_{\infty} \times \mathbb{G}_{m}}^{\mathbb{G}_{m}}$, voir (1.5), nous obtenons les égalités

$$
S_{1 / \hat{f}}\left(i\left(\mathcal{U} \backslash f^{-1}(0)\right)\right)=\sum_{I \in \mathcal{I}} S_{1 / \hat{f}}\left(i\left(E_{I}^{0} \backslash f^{-1}(0)\right)\right)=\sum_{I \in \mathcal{I}} S_{1 / \hat{f}}\left(E_{I}^{0} \backslash f^{-1}(0) \stackrel{i}{\rightarrow} X\right)
$$

Proposition 3.22. - Pour tout I dans $\mathcal{J}, S_{1 / \hat{f}}\left(E_{I}^{0} \backslash f^{-1}(0) \stackrel{i}{\rightarrow} X\right)$ est égal à $S_{g_{I}, \infty}$ avec $f_{I}$ le polynôme de Laurent de $k\left[x_{j}, x_{j}^{-1}, j \notin I\right]$ restreint au tore et égal à $f\left(\alpha_{1} x_{1}, \ldots, \alpha_{d} x_{d}\right)$, où $\alpha_{i}$ est nul pour $i$ dans $I$ et vaut 1 sinon. En particulier il ne dépend pas de la compactification.

Démonstration. - Considérons $I$ dans $\mathscr{I}$ et $(Z, E, h)$ une résolution plongée du couple $\left(\overline{i\left(E_{I}^{0} \backslash f^{-1}(0)\right)}, i\left(E_{I}^{0} \backslash f^{-1}(0)\right)\right)$. Notons $i_{Z}$ la composée $h^{-1} \circ i_{\mid E_{I}^{0} \backslash f^{-1}(0)}$. Le morphisme $h$ est propre et $i_{Z}\left(E_{I}^{0} \backslash f^{-1}(0)\right)$ est un ouvert dense de $Z$. Par le théorème $1.5, S_{1 / \hat{f}}\left(E_{I}^{0} \backslash f^{-1}(0) \stackrel{i}{\rightarrow} X\right)$ est alors égal à $h_{!} S_{1 / \hat{f} \circ h}\left(E_{I}^{0} \stackrel{i_{Z}}{\rightarrow} Z\right)$. Considérons $f_{I}$, égal à $f\left(\alpha_{1} x_{1}, \ldots, \alpha_{d} x_{d}\right)$, où $\alpha_{i}$ est nul pour $i$ dans $I$ et vaut 1 sinon. Remarquons alors que $\left(Z, i_{Z}, \hat{f} \circ h\right)$ est une compactification de $f_{I}$, en passant à l'image directe nous obtenons le résultat voulu.

Proposition 3.23. - Pour tout I dans I , le polyèdre de Newton de $f_{I}$, noté $\Gamma_{I}$, est l'intersection du polyèdre de Newton de $f$ avec $E_{I}$. Le polynôme $f_{I}$ est non dégénéré pour $\Gamma_{I}$ et commode pour $\Gamma_{I}$ si $f$ est commode pour $\Gamma$.

On applique donc la formule précédente 3.3 pour obtenir : 
ThÉORÈme 3.24. - Pour un polynôme de Laurent $f$ appartenant à $k\left[x_{1}, \ldots, x_{d}\right]\left[x_{1}^{-1}, \ldots, x_{d}^{-1}\right]$, non dégénéré pour son polyèdre de Newton $\Gamma$, la fibre de Milnor motivique à l'infini vaut

$$
S_{f, \infty}=-\sum_{I \in \mathcal{J}} \sum_{\gamma \in \Gamma_{I}^{\circ}}(-1)^{d-|I|-\operatorname{dim} \gamma}\left[\mathbb{G}_{m, I}^{d-|I|} \backslash f_{\gamma}^{-1}(0), f_{\gamma}^{-1}, \sigma_{\gamma}\right] .
$$

Si de plus $f$ est un polynôme de Laurent commode alors I est vide, $\Gamma_{\varnothing}$ est égal $\grave{a} \Gamma$ et la fibre de Milnor motivique à l'infini vaut

$$
S_{f, \infty}=-\sum_{\gamma \in \Gamma}(-1)^{d-\operatorname{dim} \gamma}\left[\mathbb{G}_{m}^{d} \backslash f_{\gamma}^{-1}(0), f_{\gamma}^{-1}, \sigma_{\gamma}\right]
$$

Si $f$ est un polynôme commode alors I est $\mathscr{P}(\{1, \ldots, d\})$ et la fibre de Milnor motivique à l'infini vaut

$$
S_{f, \infty}=-\sum_{I \in \mathcal{J}} \sum_{\gamma \in \Gamma_{I}^{\circ}}(-1)^{d-|I|-\operatorname{dim} \gamma}\left[\mathbb{G}_{m, I}^{d-|I|} \backslash f_{\gamma}^{-1}(0), f_{\gamma}^{-1}, \sigma_{\gamma, I}\right],
$$

où pour $I$ contenu dans $\mathscr{P}(\{1, \ldots, d\}), \mathbb{G}_{m, I}^{d-|I|}$ est le tore de dimension $d-|I|$ contenu dans $E_{I}, \Gamma_{I}$ et $\Gamma_{I}^{\circ}$ sont les intersections $\Gamma \cap E_{I}$ et $\Gamma^{\circ} \cap E_{I}^{0}, \sigma_{\gamma, I}$ est l'action de $\mathbb{G}_{m}$ sur $\mathbb{G}_{m, I}^{d-|I|} \backslash f_{\gamma}^{-1}(0)$ où $\sigma_{\gamma, I}(\lambda, x)$ vaut $\left(\lambda^{-\omega_{i}} x_{i}\right)$ pour $\omega$ fixé dans le cône dual de la face $\gamma$ du polyèdre $\Gamma_{I}$.

On obtient une expression du spectre à l'infini :

ThÉORÈme 3.25. - Lorsque $k$ est le corps des nombres complexes, pour un polynôme de Laurent $f$ appartenant à l'anneau $\mathbb{C}\left[x_{1}, x_{1}^{-1}, \ldots, x_{d}, x_{d}^{-1}\right]$ et non dégénéré pour $\Gamma$ son polyèdre de Newton, le spectre à l'infini de $f$ vaut

$$
\operatorname{Sp}_{f, \infty}=-\sum_{I \in \mathcal{J}} \sum_{\gamma \in \Gamma_{I}^{\circ}}(-1)^{d-|I|-\operatorname{dim} \gamma} \operatorname{Sp}\left[f_{\gamma}^{-1}(1), \sigma_{\hat{\mu}, \gamma}\right]
$$

où $\sigma_{\hat{\mu}, \gamma}$ est l'action du groupe $\hat{\mu}$ sur $f_{\gamma}^{-1}(1)$ où $\sigma_{\hat{\mu}, \gamma}\left(\lambda,\left(\underline{x_{i}}\right)\right)$ vaut $\left(\lambda^{-\omega_{i}} x_{i}\right)$ pour $\omega$ est un élément fixé du cône dual de la face $\gamma$ du polyèdre $\Gamma_{I}$.

\section{Fibres de Milnor motiviques complètes}

Soit $U$ une variété lisse sur $k$ un corps de caractéristique 0 et $f: U \rightarrow \mathbb{A}_{k}^{1}$ un morphisme dominant. 
4.1. Fibre de Milnor motivique complète. - Considérons $(X, i, \hat{f})$ une compactification de $f$. Comme en (2.1.1), pour tout $a$ dans $\mathbb{A}^{1}$ nous notons par $\hat{f}-a$ la fonction définie sur $X$ qui prolonge $f-a$.

Pour tout couple d'entiers strictement positifs $n$ et $\gamma$, pour tout $a$ dans $\mathbb{A}_{k}^{1}$, considérons l'espace d'arcs

$$
\mathscr{X}_{n}^{\gamma}(\hat{f}-a)=\left\{\begin{array}{l|l}
\varphi \in L(X) \backslash L(F) & \begin{array}{l}
\operatorname{ord}_{t} F \cdot \varphi \leq n \gamma \\
\operatorname{ord}_{t}(\hat{f}-a)(\varphi(t))=n
\end{array}
\end{array}\right\} .
$$

Cet espace d'arcs est mesurable, même si la compactification est singulière. L'ordre de contact des arcs avec le lieu singulier étant borné, sa mesure appartient à l'anneau de Grothendieck $\mathcal{M}_{X_{a} \times \mathbb{G}_{m}}^{\mathbb{G}_{m}}$ sans nécessité de complétion [10, lemme 4.1]. On considère alors la fonction zêta motivique modifiée :

$$
Z_{\hat{f}-a, U_{X}}^{\gamma}(T)=\sum_{n \geq 1} \mu\left(\mathscr{X}_{n}^{\gamma}(\hat{f}-a)\right) T^{n} \in \mathcal{M}_{X_{a} \times \mathbb{G}_{m}}^{\mathbb{G}_{m}}[[T]] .
$$

De même qu'en 2.3, on dispose de résultats de rationalité et d'indépendance :

ThÉORÈme 4.1. - Soit $f: U \rightarrow \mathbb{A}_{k}^{1}$ un morphisme à source lisse. Pour toute compactification $(X, i, \hat{f})$, lisse ou non, pour toute valeur a dans $\mathbb{A}_{k}^{1}$, et pour $\gamma$ suffisament grand, la fonction zêta modifiée $Z_{\hat{f}-a, U_{X}}^{\gamma}(T)$ est rationnelle et sa limite ne dépend pas de $\gamma$. L'opposé de cette limite est $S_{\hat{f}-a}\left(U_{X}\right)$ et appartient à l'anneau $\mathcal{M}_{X_{a} \times \mathbb{G}_{m}}^{\mathbb{G}_{m}}$. De plus, si le triplet $(Z, E, h)$ est une log-résolution $d u$ couple $\left(X, X_{a} \cup\left(X \backslash U_{X}\right)\right)$ alors $S_{\hat{f}-a, U_{X}}$ est égal à $h_{!} S_{\hat{f}-a \circ h, h^{-1}\left(U_{X}\right)}$.

THÉORÈme 4.2. - Soit $f: U \rightarrow \mathbb{A}_{k}^{1}$ un morphisme à source lisse. Pour $\left(X, i_{X}, f_{X}\right)$ et $\left(Y, i_{Y}, f_{Y}\right)$ deux compactifications et pour toute valeur a appartenant à $\mathbb{A}_{k}^{1}$, après image directe on obtient l'égalité

$$
f_{Y !} S_{f_{Y}-a, U_{Y}}=f_{X !} S_{f_{X}-a, U_{X}}=: S_{f, a} \in \mathcal{M}_{\{a\} \times \mathbb{G}_{m}}^{\mathbb{G}_{m}} .
$$

Cette valeur est indépendante de la compactification et est appelée fibre de Milnor motivique complète de $f$ pour la valeur a par opposition à $S_{f-a}$, la fibre de Milnor motivique « affine » classique de $f$ pour la valeur a.

Démonstration. - La preuve se fait de la même manière que pour le théorème 2.4.

REMARQUE 4.3. - Remarquons que pour toute compactification $\left(X, i_{X}, f_{X}\right)$, la fibre de Milnor motivique $S_{f-a}$ est égale à $i_{X}^{-1}\left(S_{f_{X}-a, U_{X}}\right)$ la restriction à l'espace affine de la fibre de Milnor motivique $S_{f_{X}-a, U_{X}}$. En effet prendre une telle restriction revient à ne considérer que des arcs dont l'origine appartient à $U$. 
4.2. Cycles évanescents motiviques à l'infini et globaux. - Par la définition des cycles évanescents motiviques 1.7 et par le théorème 4.2 , nous obtenons

Corollaire 4.4. - Soit $f: U \rightarrow \mathbb{A}_{k}^{1}$ un morphisme à source lisse. Pour $\left(X, i_{X}, f_{X}\right)$ et $\left(Y, i_{Y}, f_{Y}\right)$ deux compactifications et pour toute valeur a dans $\mathbb{A}_{k}^{1}$, on dispose de l'égalité

$$
f_{Y !} S_{f_{Y}-a, U_{Y}}^{\Phi}=f_{X !} S_{f_{X}-a, U_{X}}^{\Phi}=: S_{f, a}^{\Phi} \in \mathcal{M}_{\mathbb{G}_{m}}^{\mathbb{G}_{m}}
$$

Cette valeur est indépendante de la compactification et est appelée cycles évanescents motiviques globaux de $f$ pour la valeur a. De plus pour toute valeur a dans $\mathbb{A}_{k}^{1}$, on pose

$$
S_{f, a}^{\Phi, \infty}:=S_{f, a}^{\Phi}-f_{!} S_{f-a}^{\Phi} \in \mathcal{M}_{\mathbb{G}_{m}}^{\mathbb{G}_{m}} .
$$

Cette valeur ne dépend pas de la compactification et est appelée cycles évanescents motiviques à l'infini de $f$ pour la valeur a, par opposition à $S_{f-a}^{\Phi}$, les cycles évanescents motiviques "affines" de $f$ pour la valeur a.

REMARQUE 4.5. - Remarquons que les cycles évanescents motiviques à l'infini $S_{f, a}^{\Phi, \infty}$ s'expriment aussi comme la différence des cycles proches motiviques $S_{f, a}-f_{!} S_{f-a}$.

\subsection{Fonctions motiviquement modérées et ensemble de bifurcation motivique}

DÉFInItions 4.6. - On dit que a est une valeur motiviquement typique si et seulement si il n'existe pas de cycles évanescents motiviques à l'infini. Autrement dit, $S_{f, a}^{\Phi, \infty}$ est nul. On dit que a est motiviquement atypique dans le cas contraire. On dit que $f$ est motiviquement modérée si et seulement si toutes les valeurs de $f$ sont motiviquement typiques. On appelle ensemble de bifurcation motivique de $f$, l'union des valeurs critiques de $f$ et de l'ensemble des valeurs $a$ telles que $S_{f, a}^{\Phi}$ est non nul. On note $\mathcal{B}_{f}^{\text {mot }}$ cet ensemble.

Remarque 4.7. - Sabbah [36] et Parusiński [31] considèrent des morphismes cohomologiquement modérés. Un morphisme $f: U \rightarrow \mathbb{A}^{1}$ est cohomologiquement modéré s'il existe une extension $\hat{f}: X \rightarrow \mathbb{C}$ de $f$ avec $\hat{f}$ propre, $X$ quasi-projective, telle que pour toute valeur $c$, le support du faisceau des cycles évanescents de la fonction $\hat{f}-c$ à coefficients dans le faisceau $R i_{!} \mathbb{Q}_{U}$ ne rencontre pas $X \backslash U$ (où $i$ est l'inclusion de $U$ dans $X$ ). En particulier la restriction $i_{X \backslash F}^{-1} \psi_{1 / \hat{f}}\left(R i_{1} \mathbb{Q}_{U}\right)$ est nulle, sa classe dans le groupe de Grothendieck $K_{0}\left(D_{c}^{b}\left(\hat{f}^{-1}(c) \backslash i(U)\right)\right)$ est nulle. Notons que par le théorème de réalisation 1.8 si $f$ est cohomologiquement modéré alors pour toute valeur $c$, cette classe est nulle. On peut ainsi dire que la propriété motiviquement modéré est l'analogue motivique de la propriété cohomologiquement modéré, bien qu'il n'existe pas d'implication évidente entre ces deux notions. 
THÉORÈme 4.8. - Si $f$ est un polynôme non dégénéré et commode pour son polyèdre de Newton à l'infini alors $f$ est motiviquement modéré.

Démonstration. - Soit $f$ un polynôme appartenant à $k\left[x_{1}, \ldots, x_{d}\right]$. Rappelons que $\Gamma_{-}$est l'enveloppe convexe de $\operatorname{supp}(f) \cup\{0\}$. Nous supposons $f$ commode, autrement dit, le support de $f$ rencontre chaque axe de coordonnées. Dans ce cas là, $\Gamma_{-}$est égal à $\mathbb{Z}^{d} \backslash \mathbb{Z}_{-}^{d}$. Nous supposerons $f$ non dégénéré pour son polyèdre de Newton. Considérons pour le moment sa restriction au tore $f: \mathbb{G}_{m}^{d} \rightarrow$ $\mathbb{A}_{k}^{1}$. On montre que $S_{f, a}^{\Phi, \infty}$ est nul pour toute valeur $a$ dans $\mathbb{A}_{k}^{1}$. Remarquons que le polyèdre de Newton du polynôme ne dépend pas du terme initial, par conséquent pour deux valeurs distinctes $a$ et $b, f-a$ et $f-b$ ont le même polyèdre de Newton à l'infini. Montrons alors que 0 est motiviquement typique c'est à dire $S_{f, 0}^{\Phi, \infty}$ est nul. Pour cela reconsidérons la compactification de la partie 3 et notons $F$ le fermé à l'infini. On ne s'intéresse qu'a des $\operatorname{arcs}\left(P_{i}(t) / t^{\omega_{i}}\right)$ dont l'origine est à l'infini, par conséquent $\omega$ appartient à $\mathbb{Z}^{d} \backslash \mathbb{Z}_{-}^{d}$ et par commodité la face $\gamma(\omega)$ appartient à $\Gamma$. Notons par ce qui précède 4.5 que $S_{f, 0}^{\Phi, \infty}$ est égal à $f_{X !}\left(\lim _{T \rightarrow \infty} \sum_{n=1}^{\infty} \mu\left(\mathscr{X}_{n, \varphi(0) \in F}^{\delta}\right) T^{n}\right)$ avec pour tout couple d'entiers $n$ et $\delta$ strictement positifs

$$
\begin{aligned}
\mathscr{X}_{n, \varphi(0) \in F}^{\delta} & :=\left\{\begin{array}{l|l}
\varphi(X) & \begin{array}{l}
\operatorname{ord}_{t} \hat{f} \varphi=n \\
\varphi(0) \in F \\
\operatorname{ord}_{t} F . \varphi \leq n \delta
\end{array}
\end{array}\right\} \\
& =\left\{\begin{array}{ll}
\left(P_{i}(t) / t^{\omega_{i}}\right) & \begin{array}{l}
\operatorname{ord}_{t} f\left(P_{i}(t) / t^{\omega_{i}}\right)=n \\
\left(\omega_{i}\right) \in[-n \delta, n \delta]^{d} \backslash \mathbb{Z}_{-}^{d}
\end{array}
\end{array}\right\} .
\end{aligned}
$$

muni de l'action standard de $\mathbb{G}_{m}$ sur les arcs et de la flèche vers $X_{0} \times \mathbb{G}_{m}$ qui a un $\operatorname{arc} \varphi$ associe $(\varphi(0), \operatorname{ac} \hat{f} \varphi)$.

Nous procédons comme dans la partie 3 : nous classons tous les arcs via le polyèdre de Newton de $f$ à l'infini et par commodité, la fonction zêta s'écrit

$$
Z_{\hat{f}, U}^{\delta, \infty}(T)=\sum_{\gamma \in \Gamma} \sum_{(n, \omega) \in \mathscr{C}^{\delta} \cap \mathbb{Z}^{d+1}} \mu\left(\mathscr{X}_{n, \omega}(f)\right) T^{n}
$$

où $\mathscr{C}^{\delta}$ est le cône $\left\{n \in \mathbb{R}_{+}^{*}, \omega \in \sigma(\gamma) \mid \omega \in[-n \delta, n \delta]^{d} \backslash \mathbb{R}_{-}^{d}\right\}$ et pour $\omega$ appartenant à $\mathscr{C}^{\delta}$, l'ensemble $\mathscr{X}_{n, \omega}(f)$ est l'espace des arcs de la forme $\left(P_{i}(t) / t^{\omega_{i}}\right)$ tel que l'ordre $\operatorname{ord}_{t} f\left(P_{i}(t) / t^{\omega_{i}}\right)$ est égal à $n$. Cet ensemble est muni de la flèche « origine, coefficient angulaire » vers $X_{0} \times \mathbb{G}_{m}$ et de l'action standard de $\mathbb{G}_{m}$. Par 3.8, l'évaluation $f\left(P_{i}(t) / t^{\omega_{i}}\right)$ est égale à $t^{-m_{\Gamma}(\omega)} \tilde{f}_{\gamma}\left(P_{i}(t), t\right)$, pour tout arc $\left(P_{i}(t) / t^{\omega_{i}}\right)$. Or l'ordre de $f\left(P_{i}(t) / t^{\omega_{i}}\right)$ vaut aussi $n$, par conséquent l'ordre de $\tilde{f}_{\gamma}\left(P_{i}(t), t\right)$ vaut $m_{\Gamma}(\omega)+n$ soit de la forme $M+n$ avec $M$ strictement positif. Notons que si $f_{\gamma}\left(P_{i}(0)\right)$ est non nul, $\tilde{f}_{\gamma}\left(P_{i}(t), t\right)$ est alors d'ordre nul, ce cas là ne se produit donc pas. Par conséquent, $\left(P_{i}(0)\right)$ appartient à $f_{\gamma}^{-1}(0)$ 
noté $X_{\gamma}(0)$. Par non dégénérescence $\tilde{f}_{\gamma}$ est lisse sur $\mathbb{G}_{m}^{d} \times\{0\}$, donc par la preuve de $3.17, \mathscr{X}_{n, \omega}(f)$ est de mesure $\left[X_{\gamma}(0) \times \mathbb{G}_{m}, p, \tau_{1}\right] \mathbb{L}^{-n-m_{\Gamma}(\omega)-\sum_{i=1}^{d}\left|w_{i}\right|}$ où $p$ est une application convenable voir 3.17. Par la même preuve qu'en 3.19 le cône $C^{\delta}$ est de caractéristique d'Euler nulle. Donc par le lemme 1.2 (les conditions sur les formes linéaires étant directement vérifiées) les sommes $\sum_{(n, \omega) \in \mathscr{C}^{\delta} \cap \mathbb{Z}^{d+1}} \mu\left(\mathscr{X}_{n, \omega}(f)\right) T^{n}$ sont rationnelles de limite nulle. De la même manière qu'en 3.5 , le résultat s'étend alors par additivité à $\mathbb{A}_{k}^{d}$.

4.4. Invariant global. - Soit $(X, i, \hat{f})$ une compactification.

DÉfinition 4.9. - Pour tout couple d'entiers $n$ et $\gamma$ strictement positifs, on définit

$\mathscr{X}_{n, U_{X}}^{\gamma, \text { global }}(\hat{f}):=\left\{\varphi \in L\left(X \backslash X_{\infty}\right) \mid \operatorname{ord}_{t}(\hat{f}(\varphi(t))-\hat{f}(\varphi(0)))=n, \operatorname{ord}_{t} F . \varphi \leq n \gamma\right\}$.

On munit cet espace d'arc de l'application « origine, coefficient angulaire » vers $X \times \mathbb{G}_{m}$, qui a un arc $\varphi$ associe le couple $(\varphi(0), a c(\hat{f}(\varphi(t))-\hat{f}(\varphi(0))))$. On munit cet espace d'arcs de $\sigma$ l'action usuelle du groupe multiplicatif $\mathbb{G}_{m}$ sur les arcs. Les tronqués $\pi_{k} \mathscr{X}_{n, U_{X}}^{\gamma, \text { global }}(\hat{f})$ sont des éléments de $\operatorname{Var}_{X \times \mathbb{G}_{m}}^{\mathbb{G}_{m}}$. On considère alors la fonction zêta globale de $\hat{f}$ sur l'ouvert $U_{X}$

$$
Z_{\hat{f}, U_{X}}^{\gamma, \text { global }}(T):=\sum_{n=1}^{\infty} \mu\left(\mathscr{X}_{n, U_{X}}^{\gamma, \text { global }}(\hat{f})\right) T^{n} \in \mathcal{M}_{X \times \mathbb{G}_{m}}^{\mathbb{G}_{m}}[[T]] .
$$

On dispose du théorème de rationalité

THÉORÈme 4.10. - Soit $f: U \rightarrow \mathbb{A}_{k}^{1}$ un morphisme à source lisse et $(X, i, \hat{f})$ une compactification. On note $U_{X}$ l'ouvert dense $i(U)$. Il existe un entier $\gamma_{0}$ tel que pour tout $\gamma$ supérieur à $\gamma_{0}$, la série $Z_{\hat{f}, U_{X}}^{\gamma \text {,global }}(T)$ est rationnelle et sa limite ne dépend pas de l'entier $\gamma$. L'opposé de cette limite est noté $S G_{\hat{f}, U_{X}}$ ou $S G_{\hat{f}}$ et appartient à l'anneau $\mathcal{M}_{X \times \mathbb{G}_{m}}^{\mathbb{G}_{m}}$. On note $S G_{\hat{f}}^{\Phi}$ et on appelle cycles évanescents motiviques globaux la quantité $(-1)^{d-1}\left(S G_{\hat{f}}-\left[U_{X} \times \mathbb{G}_{m}, i d, \tau\right]\right)$ où $d$ est la dimension de la variété $X$. Pour $(Z, E, h)$ une log-résolution $d u$ couple $\left(X,\left(X \backslash U_{X}\right) \cup \hat{f}^{-1}(\operatorname{disc}(f))\right)$ on dispose de l'égalité

$$
S G_{\hat{f}}=\left[U_{X} \backslash f^{-1}\left(\mathcal{V}_{X}\right) \times \mathbb{G}_{m}, i d, \tau\right]+\sum_{a \in V_{X}} S_{\hat{f}-a, U_{X}}
$$

où $V_{X}$ est l'union du discriminant de $f$ avec les discriminants des restrictions des composées $\hat{f} \circ h$ aux strates $E_{I}^{0}$. Le symbole $\tau$ désigne ici l'action du groupe multiplicatif sur le produit $U_{X} \backslash f^{-1}\left(V_{X}\right) \times \mathbb{G}_{m}$ où pour tout $(x, \mu)$ dans $U_{X} \backslash$ $f^{-1}\left(V_{X}\right) \times \mathbb{G}_{m}$ et pour tout $\lambda$ dans $\mathbb{G}_{m}, \tau(\lambda,(x, \mu))$ est égal à $(x, \lambda \mu)$. Enfin pour tout a dans $\mathbb{A}_{k}^{1}$, la restriction $i_{X_{a}}^{-1} S G_{\hat{f}}$ vaut $S_{\hat{f}-a, U_{X}}$. 
Démonstration. - On note $F$ le fermé $X \backslash U_{X}$. Soit $(Z, E, h)$ une log-résolution du couple $\left(X, F \cup \hat{f}^{-1}(\operatorname{disc}(f))\right)$. Remarquons là encore que si $X$ est singulière son lieu singulier est contenu dans le fermé $F$ et cela n'a pas d'incidence sur la preuve. Notons $\left(E_{i}\right)_{i \in A}$ l'ensemble des composantes irréductibles (indicées par un ensemble $A$ ). On considère la stratification usuelle du diviseur : $E$ est la réunion disjointe $\bigsqcup_{\varnothing \neq I \subset A} E_{I}^{0}$ où pour toute partie non vide $I$ de $A, E_{I}^{0}$ est égal à $\bigcap_{i \in I} E_{i} \backslash \bigcup_{j \notin I} E_{j}$. Pour toute partie $I$ non vide de $A$, le discriminant de la restriction $(\hat{f} \circ h)_{\mid E_{I}^{0}}$ est un nombre fini de points. Lorsque la strate $E_{I}^{0}$ est un point, c'est à dire $I$ est égal à $d$, nous considèrerons toujours le morphisme $(\hat{f} \circ h)_{\mid E_{I}^{0}}$ comme singulier. Notons $V_{X}$ l'union du discriminant de $f$ avec les discriminants des restrictions des composées $\hat{f} \circ h$ aux strates $E_{I}^{0}$. Pour tout entier $n$ non nul on note $\mathscr{X}_{n, U_{X}, \varphi(0) \in X \backslash\left(F \cup \hat{f}^{-1}\left(V_{X}\right)\right)}^{\gamma}$ l'ensemble des arcs $\varphi$ de $\mathscr{X}_{n, U_{X}}^{\gamma}$ dont l'origine appartient à $X \backslash\left(F \cup \hat{f}^{-1}\left(V_{X}\right)\right)$. On définit de même $\mathscr{X}_{n, U_{X}, \varphi(0) \in F \backslash \hat{f}^{-1}\left(\mathcal{V}_{X}\right)}^{\gamma}$. Par additivité de la mesure, on décompose la fonction zêta comme suit

$$
\begin{aligned}
Z_{\hat{f}, U_{X}}^{\gamma, \text { global }}(T)= & \sum_{n=1}^{\infty} \mu\left(\mathscr{X}_{n, U_{X}, \varphi(0) \in X \backslash\left(F \cup \hat{f}^{-1}\left(\mathcal{V}_{X}\right)\right)}^{\gamma}\right) T^{n} \\
& +\sum_{n=1}^{\infty} \mu\left(\mathscr{X}_{n, U_{X}, \varphi(0) \in F \backslash \hat{f}^{-1}\left(\mathcal{V}_{X}\right)}^{\gamma}\right) T^{n}+\sum_{a \in V_{X}} Z_{\hat{f}-a, U_{X}}^{\gamma}(T) .
\end{aligned}
$$

Pour la première fonction zêta la restriction $\hat{f}_{\mid X \backslash\left(F \cup \hat{f}^{-1}\left(V_{X}\right)\right)}$ est une fonction lisse, de plus il n'y a pas de conditions aux bords, par conséquent cette série génératrice est rationnelle et a pour limite la classe

$$
\left[U_{X} \backslash\left(F \cup \hat{f}^{-1}\left(V_{X}\right)\right) \times \mathbb{G}_{m}, i, \tau\right]
$$

où $i$ est l'injection de $U_{X}$ dans $X$.

Montrons la rationalité de la deuxième fonction zêta et calculons sa limite. Rappelons que $h$ est un morphisme propre, par application du critère de propreté $h$ induit une bijection entre $L(Y) \backslash L(|E|)$ et $L(X) \backslash L\left(\left|F \cup \hat{f}^{-1}(\operatorname{disc}(f))\right|\right)$. Pour tout arc $\varphi$ non tracé dans $F \cup \hat{f}^{-1}(\operatorname{disc}(f))$ on note $\tilde{\varphi}$ le relevé de $\varphi$. Notons $V_{X}^{*}$ l'union $V_{X} \cup\{\infty\}$. Pour toute valeur critique $a$ appartenant à $V_{X}^{*}$, l'image inverse $(\hat{f} \circ h)^{-1}(a)$ est un diviseur à croisements normaux contenu dans $E$. Par conséquent, l'image inverse $(\hat{f} \circ h)^{-1}\left(\mathcal{V}_{X}^{*}\right)$ est un diviseur à croisements normaux contenu dans $E$. Nous travaillons ici au dessus du complémentaire de $V_{X}^{*}$, et en particulier $h^{-1}\left(F \backslash \hat{f}^{-1}\left(V_{X}^{*}\right)\right)$ est un diviseur à croisements normaux contenu dans $E$ qui ne rencontre pas $(\hat{f} \circ h)^{-1}\left(V_{X}^{*}\right)$. Notons $\mathcal{I}$ l'ensemble des parties de $A$ telles que $E_{I}^{0}$ est une strate du diviseur $h^{-1}\left(F \backslash \hat{f}^{-1}\left(V_{X}^{*}\right)\right)$. Pour tout élément non vide $I$ de $\mathscr{I}$ et pour toute famille $\left(k_{i}\right)_{i \in I}$ d'entiers strictement 
positifs introduisons alors l'espace d'arcs

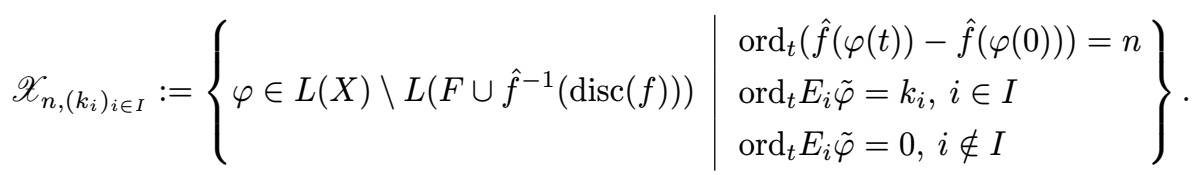

Comme $I$ appartient à $\mathscr{I}$, l'origine $\varphi(0)$ de l'arc $\varphi$ n'appartient ni à $X_{\infty}$ ni à $\hat{f}^{-1}\left(V_{X}\right)$. On obtient alors la décomposition

$$
\sum_{n \geq 1} \mu\left(\mathscr{X}_{n, U_{X}, \varphi(0) \in F \backslash \hat{f}^{-1}\left(V_{X}\right)}^{\gamma}\right) T^{n}=\sum_{\substack{\varnothing \neq I \subset A \\|I| \neq d}} \sum_{(n, \underline{k}) \in C^{\gamma} \cap \mathbb{N}^{*}|I|} \mu\left(\mathscr{X}_{n,\left(k_{i}\right)_{i \in I}}\right) T^{n}
$$

où $C^{\gamma}$ est le cône $\left\{\left(n, k_{i}\right) \in \mathbb{R}^{*|I|+1} \mid \sum_{i \in I} k_{i} N_{i}\left(\mathcal{J}_{F}\right) \leq n \gamma\right\}$, où les coefficients $N_{i}\left(J_{F}\right)$ sont définis par le diviseur $h^{-1}(F)$ égal à $\sum_{i \in A} N_{i}\left(J_{F}\right) E_{i}$ et sont strictement positifs pour tout $i$ appartenant à un ensemble d'indices $I$ contenu dans I (car chaque strate associée $E_{I}^{0}$ est contenue dans le diviseur à croisements normaux $\left.h^{-1}\left(F \backslash \hat{f}^{-1}\left(\mathcal{V}_{X}^{*}\right)\right)\right)$.

Calculons la mesure de $\mathscr{X}_{n,\left(k_{i}\right)_{i \in I}}$. Pour cela on note $\mathscr{Y}_{n,\left(k_{i}\right)_{i \in I}}$ l'image inverse $h^{-1}\left(\mathscr{X}_{n,\left(k_{i}\right)_{i \in I}}\right)$. On obtient alors l'égalité

$$
\mathscr{Y}_{n,\left(k_{i}\right)_{i \in I}}=\left\{\begin{array}{l|l}
\varphi \in L(Y) \backslash L(E) & \begin{array}{l}
\operatorname{ord}_{t}(\hat{f}(h(\varphi(t)))-\hat{f}(h(\varphi(0))))=n \\
\operatorname{ord}_{t} E_{i} \varphi=k_{i}, i \in I \\
\operatorname{ord}_{t} E_{i} \varphi=0, i \notin I
\end{array}
\end{array}\right\} .
$$

Comme $I$ appartient à $\mathcal{I}$, l'image $h(\varphi(0))$ n'appartient ni à $X_{\infty}$ ni $\hat{f}^{-1}\left(\mathcal{V}_{X}\right)$. On note $\pi_{n \gamma}$ le morphisme de troncation des arcs à l'ordre $n \gamma$. Dans la catégorie $\operatorname{Var}_{X \times \mathbb{G}_{m}}^{\mathbb{G}_{m}}$, pour toute partie $I$ appartenant à $\mathscr{f}$ de cardinal strictement inférieur à $d$, le morphisme de $\pi_{n \gamma}\left(\mathscr{Y}_{\left.n,\left(k_{i}\right)_{i \in I}\right)}\right)$ vers $E_{I}^{0} \times \mathbb{G}_{m}$, qui a un arc $\varphi$ associe le couple $(\varphi(0), \operatorname{ac}(\hat{f} \circ h(\varphi(t))-\hat{f} \circ h(\varphi(0))))$ est une fibration localement triviale de fibre $\mathbb{A}^{n \gamma d-\sum_{i \in I} k_{i}-n} \times \mathbb{G}_{m}^{|I|}$. En effet, soit $y$ appartenant à $E_{I}^{0}$ et $\left(y_{i}\right)_{i \in A}$ des équations locales des composantes irréductibles $E_{i}$ du diviseur au voisinage de $y$. Ces équations forment une suite régulière et par hypothèse $\hat{f}(h(y))$ n'appartient pas au discriminant de la restriction de $\hat{f} \circ h$ à la strate $E_{I}^{0}$. Par conséquent, la famille $\left(y_{i}\right)_{i \in I}, f \circ h$ peut être complétée en une application étale $\Phi$ définie sur un ouvert $\Omega$ de $Y$ car $Y$ est lisse en $y$ et le cardinal de $I$ est strictement inférieur à $d$. Ordonnons les éléments de $I$, et notons les $\left(i_{k}\right)_{k \in\{1, \ldots,|I|\}}$. Par application du lemme $[9,4.2], \mathscr{Y}_{n,\left(k_{i}\right)_{i \in I}}$ est alors isomorphe au produit fibré

$$
\begin{aligned}
& \left(\Omega \cap E_{I}^{0}\right) \times_{\mathbb{A}^{d}}\left\{\begin{array}{l|l}
\psi \in L_{n \gamma}\left(\mathbb{A}^{d}\right) & \begin{array}{l}
\psi_{j}(t)=a_{j} t^{k_{i_{j}}}+\cdots+* t^{n \gamma}, j \in\{1, \ldots,|I|\} \\
\psi_{|I|+1}(t)=\psi_{|I|+1}(0)+a_{|I|+1} t^{n}+\cdots+* t^{n \gamma}
\end{array}
\end{array}\right\} \\
& \text { TOME } 140-2012-\mathrm{N}^{\mathrm{O}} 1
\end{aligned}
$$


où les $a_{i}$ sont non nuls et les images $\Phi(y)$ et $\psi(0)$ sont égales pour deux éléments $(y, \psi)$ du but. Celui-ci est muni d'une action de $\mathbb{G}_{m}$ qui a $(\lambda,(y, \psi(t)))$ associe $(y, \psi(\lambda t))$ et d'une flèche vers $X \times \mathbb{G}_{m}$ à fibres $\mathbb{G}_{m}$-invariantes qui a $(y, \psi)$ associe $a c\left(\psi_{|I|+1}(t)-\psi_{|I|+1}(0)\right)$. On déduit de cela la fibration dans la catégorie $\operatorname{Var}_{X \times \mathbb{G}_{m}}^{\mathbb{G}_{m}}$. Par conséquent la mesure de $\mathscr{Y}_{n,\left(k_{i}\right)_{i \in I}}$ vaut $\left[E_{I}^{0} \times \mathbb{G}_{m}, i d, \tau\right] \mathbb{L}^{-\sum_{i \in I} k_{i}-n}$ dans l'anneau $\mathcal{M}_{Y \times \mathbb{G}_{m}}^{\mathbb{G}_{m}}$ et par application de la formule de changement de variables de Denef et Loeser [10, 3.3], la mesure $\mu\left(\mathscr{X}_{n,\left(k_{i}\right)_{i \in I}}\right)$ vaut $\left[E_{I}^{0} \times \mathbb{G}_{m}, i d, \tau\right] \mathbb{L}^{-\sum_{i \in I} \nu_{i} k_{i}-n}$ où les $\nu_{i}$ sont définis par l'égalité $\operatorname{Jac}(h)=\sum_{i \in A}\left(\nu_{i}-1\right) E_{i}$.

Enfin nous sommes amenés à montrer la rationalité de la série $\sum_{(n, \underline{k}) \in C^{\gamma \cap \mathbb{N}^{*}|I|+1}} \mathbb{L}^{-\sum_{i \in I} k_{i}-n} T^{n}$. L'ensemble d'indices $I$ est contenu dans $\mathscr{J}$ donc $N_{i}\left(\mathscr{I}_{F}\right)$ est strictement positif. Le complémentaire du cône $C^{\gamma}$ polyédral rationnel convexe dans $\mathbb{R}^{*|I|+1}$ est défini par l'inégalité $\sum_{i \in I} k_{i} N_{i}\left(\mathcal{I}_{F}\right)>n \gamma$. Il est donc relativement ouvert et de caractéristique d'Euler à support compact égale à $(-1)^{d+1}$. Par additivité de la caractéristique d'Euler, le cône $C^{\gamma}$ est de caractéristique d'Euler nulle. La forme linéaire $l$ qui associe $n$ à chaque couple $(n, \underline{k})$ est strictement positive sur $\overline{C^{\gamma}} \backslash\{0\}$. En effet les coefficients $N_{i}\left(\mathscr{I}_{F}\right)$ sont strictement positifs donc par prolongement de l'inégalité définissant le cône, par passage à la limite, la nullité de $n$ entraîne la nullité des $k_{i}$. La forme linéaire qui associe $-\sum_{i \in I} \nu_{i} k_{i}-n$ à tout couple $(n, \underline{k})$ du cône $C^{\gamma}$ est elle aussi strictement positive sur $\overline{C^{\gamma}} \backslash\{0\}$. Par application du lemme 1.2, la série $\sum_{(n, \underline{k}) \in C^{\gamma \cap \mathbb{N}^{*}|I|+1}} \mathbb{L}^{-\sum_{i \in I} k_{i}-n} T^{n}$ est rationnelle et de limite nulle.

Enfin pour toute valeur $a$ dans $V_{X}$ la fonction zêta $Z_{\hat{f}-a, U_{X}}^{\gamma}(T)$ est rationnelle. En effet, le lieu singulier de $X$ est contenu dans le fermé à l'infini $F$, on peut donc agir comme en 2.3. L'existence de $\gamma_{0}$ vient de ce théorème de rationalité. Pour toute valeur $a$ dans $\mathbb{A}_{k}^{1}$, on déduit des définitions l'égalité $i_{X_{a}}^{-1} S G_{\hat{f}}=S_{\hat{f}-a, U_{X}}$.

On dispose alors du théorème d'indépendance suivant :

ThÉORÈme 4.11. - Soit $f: U \rightarrow \mathbb{A}_{k}^{1}$ un morphisme à source lisse. Pour deux compactifications $\left(X, i_{X}, f_{X}\right)$ et $\left(Y, i_{Y}, f_{Y}\right)$, les objets $f_{X !} S G_{f_{X}}$ et $f_{Y !} S G_{f_{Y}}$ sont égaux dans $\mathcal{M}_{\mathbb{A}_{k}^{1} \times \mathbb{G}_{m}}^{\mathbb{G}_{m}}$. On note $S G_{f}$ cet invariant et on l'appelle invariant global de $f$. Ceci induit l'égalité entre $f_{X !} S G_{f_{X}}^{\Phi}$ et $f_{Y !} S G_{f_{Y}}^{\Phi}$. On note $S G_{f}^{\Phi}$ cet invariant et on l'appelle cycles évanescents motiviques globaux de $f$. Enfin en notant $i_{a}$ l'injection du singleton $\{a\}$ dans la droite $\mathbb{A}_{k}^{1}$ pour tout a dans $\mathbb{A}_{k}^{1}$, les objets $i_{a}^{-1} S G_{f}$ et $S_{f, a}$ sont égaux tout comme $i_{a}^{-1} S G_{f}^{\Phi}$ et $S_{f, a}^{\Phi}$.

Démonstration. - Soit $\left(X, i_{X}, f_{X}\right)$ et $\left(Y, i_{Y}, f_{Y}\right)$ deux compactifications. Les immersions $i_{X}$ et $i_{Y}$ induisent chacune un isomorphisme entre $X \backslash\left(F_{X} \cup\right.$ $\left.f_{X}^{-1}(\operatorname{disc} f)\right)$ et $Y \backslash\left(F_{Y} \cup f_{Y}^{-1}(\operatorname{disc} f)\right)$ avec l'ouvert $U \backslash f^{-1}(\operatorname{disc}(f))$. On applique 
alors le théorème de coiffage 2.8 à $\left(X, F_{X} \cup f_{X}^{-1}(\operatorname{disc} f)\right)$ et $\left(Y, F_{Y} \cup f_{Y}^{-1}(\operatorname{disc} f)\right)$. On obtient ainsi un quadruplet $\left(Z, E, h_{X}, h_{Y}\right)$ tel que $\left(Z, E, h_{X}\right)$ est une logrésolution de $\left(X, F_{X} \cup f_{X}^{-1}(\operatorname{disc} f)\right)$ et $\left(Z, E, h_{Y}\right)$ est une log-résolution de $\left(Y, F_{Y} \cup f_{Y}^{-1}(\operatorname{disc} f)\right)$. En particulier $f_{X} \circ h_{X}$ est égal à $f_{Y} \circ h_{Y}$. Par le théorème précédent $f_{X !} S G_{f_{X}}$ est égal à $f_{Y !} S G_{f_{Y}}$. L'égalité évanescente en découle.

DÉFInition 4.12. - Soit $f: U \rightarrow \mathbb{A}^{1}$ une fonction à source lisse. On appelle discriminant universel et on note $\mathscr{D}_{f}$, l'ensemble formé du discriminant de $f$ et des valeurs $a$ telles que pour toute compactification $(X, i, \hat{f})$, pour toute logrésolution $(Z, E, h)$ du couple $\left(X, F \cup \hat{f}^{-1}(\operatorname{disc}(f))\right)$, il existe une strate $E_{I}^{0}$ du diviseur pour laquelle $a$ appartient au discriminant de $(\hat{f} \circ h)_{\mid E_{I}^{0}}$.

ThÉORÈme 4.13. - Soit $f: U \rightarrow \mathbb{A}_{k}^{1}$ un morphisme à source lisse. L'ensemble de bifurcation motivique est contenu dans le discriminant universel $\mathscr{D}_{f}$. De plus on dispose de l'égalité

$$
S G_{f}^{\Phi}=\sum_{a \in B_{f}^{\text {mot }}} S_{f, a}^{\Phi} \in \mathcal{M}_{\mathbb{A}_{k}^{1} \times \mathbb{G}_{m}}^{\mathbb{G}_{m}}
$$

Les cycles évanescents motiviques globaux sont supportés par l'ensemble de bifurcation motivique.

Démonstration. - Notons que $\mathscr{D}_{f}$ est l'intersection $\bigcap_{\left(X, h_{X}\right)} V_{X}$. Cette intersection est faite sur l'ensemble des compactifications $(X, i, \hat{f})$ de $f$ et sur l'ensemble des log-résolutions de $\left(X, F \cup \hat{f}^{-1}(\operatorname{disc}(f))\right)$; avec $V_{X}$ l'union du discriminant de $f$ avec les discriminants des restrictions des composées $\hat{f} \circ h$ aux strates $E_{I}^{0}$. Par indépendance des compactifications et des log-résolutions et par conditions de supports, les cycles évanescents motiviques globaux $S G_{f}^{\Phi}$ se décomposent dans $\mathcal{M}_{\mathbb{A}_{k}^{2} \times \mathbb{G}_{m}}^{\mathbb{G}_{m}}$ sous la forme $\sum_{a \in \mathscr{D}_{f} \cap B_{f}^{\text {mot }}} S_{f, a}^{\Phi}$. En effet, pour deux compactifications $\left(X, i_{X}, f_{X}\right)$ et $\left(Y, i_{Y}, f_{Y}\right)$, deux log-résolutions $\left(X^{\prime}, E_{X}^{\prime}\right)$ et $\left(Y^{\prime}, E_{Y}^{\prime}\right)$ des couples $\left(X, F_{X} \cup f_{X}^{-1}(\operatorname{disc}(f))\right)$ et $\left(Y, F_{Y} \cup f_{Y}^{-1}(\operatorname{disc}(f))\right)$ le théorème d'indépendance montre que $S G_{f}^{\Phi}, f_{X !} \sum_{a \in V_{X}} S_{f_{X}-a, U_{X}}^{\Phi}$ et $f_{Y !} \sum_{a \in V_{Y}} S_{f_{Y}-a, U_{y}}^{\Phi}$ sont égaux dans $\mathcal{M}_{\mathbb{A}_{k}^{1} \times \mathbb{G}_{m}}^{\mathbb{G}_{m}}$. Notons alors que pour toute valeur $a$ appartenant à l'union $V_{X} \cup V_{Y}$ mais pas à l'intersection $V_{X} \cap V_{Y}$ les objets $i_{a}^{-1} S G_{f}^{\Phi}, f_{X !} S_{f_{X}-a, U_{X}}^{\Phi}$ et $f_{Y !} S_{f_{Y}-a, U_{Y}}^{\Phi}$ sont égaux dans l'anneau $\mathcal{M}_{\mathbb{G}_{m}}^{\mathbb{G}_{m}}$. Or $a$ n'appartient pas à l'intersection $V_{X} \cap V_{Y}$, par conséquent l'un des membres de l'égalité est nulle.

Si une valeur $a$ n'appartient pas à $\tilde{B}$ alors il existe une compactification $(X, i, \hat{f})$ et une log-résolution $(Z, E, h)$ de $\left(X, F \cup \hat{f}^{-1}(\operatorname{disc}(f))\right)$ telles que pour toutes strates $E_{I}^{0}, a$ n'est pas une valeur critique de la resctriction $\left(f_{X} \circ h\right)_{\mid E_{I}^{0}}$. En ce cas $a$ n'appartient pas à $V_{X}$ et par ce qui précède $f_{X !} S_{f_{X}-a, U_{X}}^{\Phi}$ est nul. Or $f_{X !} S_{f_{X}-a, U_{X}}^{\Phi}$ est égal à $S_{f, a}^{\Phi}$ donc $S_{f, a}^{\Phi}$ est nul. Le discriminant de $f$ est 
contenu dans $\mathscr{D}_{f}$. Par conséquent, pour toute valeur $a$ n'appartenant pas à $\mathscr{D}_{f}, S_{f-a}^{\Phi}$ est nul, $S_{f, a}^{\Phi, \infty}$ est alors nul et $a$ n'est pas motiviquement atypique. Ainsi, l'ensemble de bifurcation motivique est contenu dans $\mathscr{D}_{f}$.

THÉORÈme 4.14. - Soit $f: U \rightarrow \mathbb{A}_{k}^{1}$ un morphisme à source lisse. Si $f$ est motiviquement modéré alors l'ensemble de bifurcation motivique est égal au discriminant de $f$.

Démonstration. - En effet, si $f$ est motiviquement modéré, les cycles évanescents motiviques de $f$ sont alors supportés par l'ouvert lisse $U$. Or dans l'ouvert lisse $U$ il n'y a existence de cycles évanescents motiviques que là où $f$ n'est pas lisse. Par conséquent si $f$ est motiviquement modéré l'ensemble de bifurcation motivique contient et est contenu dans le discriminant de $f$, il lui est donc égal.

On obtient alors directement le théorème

THÉORÈme 4.15. - Soit $f: U \rightarrow \mathbb{A}_{k}^{1}$ un morphisme à source lisse. Si $f$ est motiviquement modéré alors

$$
S G_{f}^{\Phi}=\sum_{a \in \operatorname{disc}(f)} p_{!}\left(S_{f-a}^{\Phi}\right) .
$$

Si $f$ est à singularités isolées et motiviquement modéré alors

$$
S G_{f}^{\Phi}=\sum_{a \in \operatorname{disc}(f)} \sum_{x \in \operatorname{Sing}\left(f^{-1}(a)\right)}\left(S_{f-a}^{\Phi}\right)_{x} .
$$

REMARQUe 4.16. - Les formules obtenues dans cette partie sont des analogues motiviques de la formule d'Iversen [21], on pourra consulter à ce propos $[13,6.2 .5]$.

REMARQUE 4.17. - Kouchnirenko [22], puis Broughton [4], [5] ont montré que l'ensemble de bifurcation d'un polynôme $f$, commode et non dégénéré pour son polyèdre de Newton à l'infini est le discriminant de $f$. Dans ce cas là l'ensemble de bifurcation motivique et l'ensemble de bifurcation sont égaux.

\subsection{Cas d'un polynôme homogène}

Proposition 4.18. - Soit $f$ un polynôme homogène en $d$ variables. L'ensemble de bifurcation motivique $\mathcal{B}_{f}^{\text {mot }}$ est réduit à $\{0\}$. 
Démonstration. - On peut considérer la compactification $(X, i, \hat{f})$ de $f$ dans $\mathbb{P}^{d} \times \mathbb{P}^{1}$

$$
X=\left\{\left[x_{0}: \cdots: x_{d}\right],[\alpha: \beta] \mid \alpha f\left(x_{1}, \ldots, x_{d}\right)=\beta x_{0}^{\operatorname{deg}(f)}\right\}
$$

avec $i$ l'injection $\left(x_{1}, \ldots, x_{d}\right) \mapsto\left(\left[1: x_{1}: \ldots: x_{d}\right],\left[1: f\left(x_{1}, \ldots, x_{d}\right)\right]\right)$ et $\hat{f}$ la projection de $X$ sur $\mathbb{P}^{1}$. Par homogénéité de $f$ et la relation d'Euler, 0 est l'unique valeur critique de $f$, et si une valeur $a$ non nulle est motiviquement atypique alors par homogénéité toutes les valeurs sont motiviquement atypiques. Or l'ensemble de bifurcation motivique est un ensemble fini ce qui entraîne une contradiction.

Dans le cas homogène nous pouvons relier la fibre de Milnor motivique complète en 0 et la fibre de Milnor motivique à l'infini comme suit :

DÉfinition 4.19. - Notons inv l'opération sur $\mathcal{M}_{\mathbb{G}_{m}}^{\mathbb{G}_{m}}$ qui a une classe $[A, p, \sigma]$ associe la classe $[A$, inv $\circ p, \sigma]$ induite par l'inversion sur $\mathbb{G}_{m}$ notée encore inv qui à $x$ associe $x^{-1}$.

ThÉorème 4.20. - Pour $f$ un polynôme homogène $S_{f, 0}$ et $\operatorname{inv}\left(S_{f, \infty}\right)$ sont égaux dans $\mathcal{M}_{\mathbb{G}_{m}}^{\mathbb{G}_{m}}$.

Démonstration. - Nous calculons la fibre de Milnor motivique à l'infini du polynôme homogène $f$ et sa fibre de Milnor motivique complète au dessus de la valeur 0 , avec la compactification $(X, i, \hat{f})$ précédente. Pour tout couple d'entiers $(\gamma, n)$ strictement positifs on considére

$$
\mathscr{X}_{n}^{\gamma}(\hat{f}, 0)=\left\{\begin{array}{l|c}
{\left[\underline{x_{i}(t)}\right],[\alpha(t): \beta(t)]} & \begin{array}{c}
\alpha(t) \\
\underline{\left.x_{j}(t)\right)}=\beta(t) x_{0}(t)^{\operatorname{deg}(f)} \\
\operatorname{ord}_{t} \beta(t) / \alpha(t)=n \\
\operatorname{ord}_{t} x_{0}(t) \leq n \gamma
\end{array}
\end{array}\right\}
$$

On munit cet espace d'arcs de l'action usuelle de $\mathbb{G}_{m}$ sur les arcs, $\sigma(\lambda, \varphi)$ égal à $\varphi(\lambda t)$ et de l'application «origine, coefficient angulaire». Pour tout $k$, les tronqués $\pi_{k} \mathscr{X}_{n}^{\gamma}(\hat{f}, 0)$ sont des objets de la catégorie $\operatorname{Var}_{X \times \mathbb{G}_{m}}^{\mathbb{G}_{m}}$. Pour tout couple d'entiers $(\gamma, n)$ strictement positifs on considère

$$
\mathscr{X}_{n(2 \operatorname{deg}(f)-1)}^{\gamma}(\hat{f}, \infty)=\left\{\begin{array}{l|c}
{\left[\underline{\left.x_{i}(t)\right]},[\alpha(t): \beta(t)]\right.} & \begin{array}{c}
\alpha(t) f\left(x_{j}(t)\right)=\beta(t) x_{0}(t)^{\operatorname{deg}(f)} \\
\operatorname{ord}_{t} \alpha(t) / \beta(t)=n(2 \operatorname{deg}(f)-1) \\
\operatorname{ord}_{t} x_{0}(t) \leq n(\gamma+2)
\end{array}
\end{array}\right\}
$$

On munit cet espace d'arcs de l'action usuelle de $\mathbb{G}_{m}$ sur les arcs, $\sigma(\lambda, \varphi)$ égal à $\varphi(\lambda t)$ et de l'application « origine, coefficient angulaire ». Pour tout $k$, ses tronqués sont des objets de la catégorie $\operatorname{Var}_{X \times \mathbb{G}_{m}}^{\mathbb{G}_{m}}$. Notons que pour tout polynôme $f, 2 \operatorname{deg}(f)-1$ est un entier strictement positif. Pour tout arc $\left(\left[x_{i}(t)\right],[\alpha(t): \beta(t)]\right)$ appartenant à $\mathscr{X}_{n}^{\gamma}(\hat{f}, 0), \beta(0)$ est nul, $\alpha(0)$ est non nul et 
l'arc $\beta(t) / \alpha(t)$ est égal à $f\left(\left(x_{j}(t) / x_{0}(t)\right)\right)$. De même pour tout arc $\left(\left[x_{i}(t)\right],[\alpha(t)\right.$ : $\beta(t)])$ appartenant à $\mathscr{X}_{n(2 \operatorname{deg}(f)-1)}^{\gamma}(\hat{f}, \infty)$, l'origine $\alpha(0)$ est nulle, $\beta(0)$ est non nulle et l'arc $\alpha(t) / \beta(t)$ est égal à $f\left(\left(x_{j}(t) / x_{0}(t)\right)\right)^{-1}$.

REMARQUe 4.21. - Rappelons que la fonction zêta modifiée de $f$ en 0 par rapport à l'ouvert $U_{X}$, notée $Z_{f, 0, U_{X}}^{\gamma}(T)$, est la série génératrice $\sum_{n \geq 1} \mu\left(\mathscr{X}_{n}^{\gamma}(\hat{f}, 0)\right) T^{n}$. Cette série est rationnelle et converge quand $T$ tend vers l'infini vers $-S_{\hat{f}, U_{X}}$. Considérons la série génératrice $\sum_{n \geq 1} \mu\left(\mathscr{X}_{n(2 \operatorname{deg}(f)-1)}^{\gamma}(\hat{f}, \infty)\right) T^{n(2 \operatorname{deg}(f)-1)}$, elle est légèrement différente de la série $Z_{f, \infty, U_{X}}^{\gamma}(T)$ égale à $\sum_{n \geq 1} \mu\left(\mathscr{X}_{n}^{\gamma}(\hat{f}, \infty)\right) T^{n}$ mais la preuve de (3.8) [19] montre que ces deux séries sont rationnelles et convergent vers la même limite $-S_{\hat{f}, \infty}$. L'argument clé étant que les cônes utilisés ont la même caractéristique d'Euler.

Lemme 4.22. - Pour tout couple d'entiers $(\gamma, n)$ strictement positifs,

$$
\begin{aligned}
& \Phi: \mathscr{X}_{n}^{\gamma}(\hat{f}, 0) \rightarrow \operatorname{inv}\left(\mathscr{X}_{2 n(\operatorname{deg}(f)-1)}^{\gamma}(\hat{f}, \infty)\right) \\
&\left(\underline{\left.\left[x_{i}(t)\right],\left[x_{0}(t)^{\operatorname{deg}(f)}: f\left(\underline{x_{j}(t)}\right)\right]\right)} \mapsto\left(\left[t^{2 n} x_{0}(t): \underline{x_{j}(t)}\right],\left[\left(t^{2 n} x_{0}(t)\right)^{\operatorname{deg}(f)}: f\left(\underline{x_{i}(t)}\right)\right]\right)\right.
\end{aligned}
$$

est un isomorphisme dans la catégorie $\operatorname{Var}_{X \times \mathbb{G}_{m}}^{\mathbb{G}_{m}}$

Démonstration. - Par homogénéité de $f$, on vérifie que $\Phi$ est bien définie et inversible, d'inverse

$$
\begin{aligned}
\Psi: \operatorname{inv}\left(\mathscr{X}_{n(2 \operatorname{deg}(f)-1), U_{X}}^{\gamma}(\hat{f}, \infty)\right) & \rightarrow \mathscr{X}_{n, U_{X}}^{\gamma}(\hat{f}, 0) \\
\left(\left[\underline{\left.x_{i}(t)\right]}\right],\left[x_{0}(t)^{\operatorname{deg}(f)}: f\left(\underline{x_{i}(t)}\right)\right]\right) & \mapsto\left(\left[x_{0}(t): \underline{t^{2 n} x_{i}(t)}\right],\left[\left(x_{0}(t)\right)^{\operatorname{deg}(f)}: f\left(\underline{t^{2 n} x_{i}(t)}\right)\right]\right)
\end{aligned}
$$

Rappelons que $\left[t^{2 n} x_{i}(t)\right]=\left[\underline{x_{i}}\right]$. Pour tout $\operatorname{arc} \varphi,\left(\operatorname{inv} \circ p_{\mathbb{G}_{m}}\right) \Phi(\varphi)$ est bien

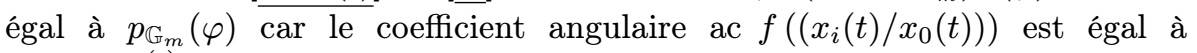
$\left(\operatorname{ac}\left(f\left(\frac{x_{i}(t)}{t^{n} x_{0}(t)}\right)^{-1}\right)\right)^{-1}$. On vérifie directement la compatibilité de $\Phi$ avec l'action $\sigma$ : pour tout scalaire non nul $\lambda$, pour tout $\operatorname{arc} \varphi, p_{\mathbb{G}_{m}}(\sigma(\lambda, \varphi))$ est égal à (inv $\left.\circ p_{\mathbb{G}_{m}} \circ \Phi\right)(\sigma(\lambda, \varphi))$.

Par conséquent, pour tout couple $(n, \gamma)$ d'entiers strictement positifs, on obtient dans l'anneau $\mathcal{M}_{X \times \mathbb{G}_{m}}^{\mathbb{G}_{m}}$, l'égalité des mesures $\mu\left(\mathscr{X}_{n}^{\gamma}(\hat{f}, 0)\right)$ et $\mu\left(\operatorname{inv}\left(\mathscr{X}_{n(2 \operatorname{deg}(f)-1)}^{\gamma}(\hat{f}, \infty)\right)\right)$. D'où l'expression de la fonction zêta $Z_{f, 0, U_{X}}^{\gamma}\left(T^{2 \operatorname{deg}(f)-1}\right)$ et $\sum_{n \geq 1} \mu\left(\operatorname{inv}\left(\mathscr{X}_{n(2 \operatorname{deg}(f)-1), U_{X}}^{\gamma}(\hat{f}, \infty)\right)\right) T^{n(2 \operatorname{deg}(f)-1)} \cdot \operatorname{Par}$ la remarque 4.21 et l'égalité des limites $\lim _{T \rightarrow \infty} Z_{f, 0, U_{X}}^{\gamma}(T)$ et $\lim _{T \rightarrow \infty} Z_{f, 0, U_{X}}^{\gamma}\left(T^{2 \operatorname{deg}(f)-1}\right)$ en passant à l'image directe on obtient l'égalité des fibres de Milnor motiviques cherchée. 


\section{BIBLIOGRAPHIE}

[1] F. BitTneR - « On motivic zeta functions and the motivic nearby fiber », Math. Z. 249 (2005), p. 63-83.

[2] T. BRÉLIVET - «Variance of the spectral numbers and Newton polygons », Bull. Sci. Math. 126 (2002), p. 332-342.

[3] _ , « Sur les paires spectrales de polynômes à deux variables », in Singularités Franco-Japonaises, Sémin. Congr., vol. 10, Soc. Math. France, 2005, p. 39-59.

[4] S. A. Broughton - « On the topology of polynomial hypersurfaces », in Singularities, Part 1 (Arcata, Calif., 1981), Proc. Sympos. Pure Math., vol. 40, Amer. Math. Soc., 1983, p. 167-178.

[5] _ « Milnor numbers and the topology of polynomial hypersurfaces », Invent. Math. 92 (1988), p. 217-241.

[6] P. Cassou-Noguès \& A. Dimca - « Topology of complex polynomials via polar curves », Kodai Math. J. 22 (1999), p. 131-139.

[7] B. ConRAD - « Deligne's notes on Nagata compactifications », J. Ramanujan Math. Soc. 22 (2007), p. 205-257.

[8] P. Deligne - « Théorie de Hodge. III », Publ. Math. I.H.É.S. 44 (1974), p. $5-77$.

[9] J. Denef \& F. Loeser - «Motivic Igusa zeta functions », J. Algebraic Geom. 7 (1998), p. 505-537.

[10] _ « Germs of arcs on singular algebraic varieties and motivic integration », Invent. Math. 135 (1999), p. 201-232.

[11] _ « Geometry on arc spaces of algebraic varieties », in European Congress of Mathematics, Vol. I (Barcelona, 2000), Progr. Math., vol. 201, Birkhäuser, 2001, p. 327-348.

[12] A. DimcA - «Monodromy and Hodge theory of regular functions », in New developments in singularity theory (Cambridge, 2000), NATO Sci. Ser. II Math. Phys. Chem., vol. 21, Kluwer Acad. Publ., 2001, p. 257-278.

[13] _ Sheaves in topology, Universitext, Springer, 2004.

[14] R. García López \& A. NÉmethi - « On the monodromy at infinity of a polynomial map », Compositio Math. 100 (1996), p. 205-231.

[15] « Hodge numbers attached to a polynomial map », Ann. Inst. Fourier (Grenoble) 49 (1999), p. 1547-1579.

[16] _ « On the monodromy at infinity of a polynomial map. II », Compositio Math. 115 (1999), p. 1-20.

[17] G. GuiberT - «Espaces d'arcs et invariants d'Alexander », Comment. Math. Helv. 77 (2002), p. 783-820. 
[18] G. Guibert, F. Loeser \& M. Merle - « Nearby cycles and composition with a nondegenerate polynomial », Int. Math. Res. Not. 2005 (2005), p. $1873-1888$.

[19] _ « Iterated vanishing cycles, convolution, and a motivic analogue of a conjecture of Steenbrink », Duke Math. J. 132 (2006), p. 409-457.

[20] H. HironakA - « Resolution of singularities of an algebraic variety over a field of characteristic zero. I, II », Ann. of Math. 79 (1964), p. 109-203, 205-326.

[21] B. IVERSEN - « Critical points of an algebraic function », Invent. Math. 12 (1971), p. 210-224.

[22] A. G. Kouchnirenko - « Polyèdres de Newton et nombres de Milnor », Invent. Math. 32 (1976), p. 1-31.

[23] F. Loeser - «Seattle lectures on motivic integration », in Algebraic geometry-Seattle 2005. Part 2, Proc. Sympos. Pure Math., vol. 80, Amer. Math. Soc., 2009, p. 745-784.

[24] E. Looijenga - « Motivic measures », Astérisque 276 (2002), p. 267-297, Séminaire Bourbaki, vol. 1999/2000, exp. n 874 .

[25] Y. Matsui \& K. TAKeUChi - « Monodromy at infinity of polynomial map and mixed hodge modules $\gg$.

[26] _ _ «onodromy zeta functions at infinity, newton polyhedra and constructible sheaves », prépublication arXiv:0809.3149.

[27] A. Némethi \& C. SABbAh - «Semicontinuity of the spectrum at infinity », Abh. Math. Sem. Univ. Hamburg 69 (1999), p. 25-35.

[28] A. NÉmethi \& A. Zaharia - « On the bifurcation set of a polynomial function and Newton boundary », Publ. Res. Inst. Math. Sci. 26 (1990), p. 681-689.

[29] _ « Milnor fibration at infinity», Indag. Math. (N.S.) 3 (1992), p. 323-335.

[30] A. PARUSIŃSKI - «On the bifurcation set of complex polynomial with isolated singularities at infinity », Compositio Math. 97 (1995), p. 369384 .

[31] _ « A note on singularities at infinity of complex polynomials », in Symplectic singularities and geometry of gauge fields (Warsaw, 1995), Banach Center Publ., vol. 39, Polish Acad. Sci., 1997, p. 131-141.

[32] C. A. M. Peters \& J. H. M. Steenbrink - Mixed Hodge structures, Ergebn. Math. Grenzg., vol. 52, Springer, 2008.

[33] F. PHAM - «Vanishing homologies and the $n$ variable saddlepoint method », in Singularities, Part 2 (Arcata, Calif., 1981), Proc. Sympos. Pure Math., vol. 40, Amer. Math. Soc., 1983, p. 319-333. 
[34] M. Raibaut - «Fibre de Milnor motivique à l'infini », C. R. Math. Acad. Sci. Paris 348 (2010), p. 419-422.

[35] C. SabBah - «Monodromy at infinity and Fourier transform », Publ. Res. Inst. Math. Sci. 33 (1997), p. 643-685.

[36] _ «ypergeometric periods for a tame polynomial», Port. Math. (N.S.) 63 (2006), p. 173-226.

[37] , « Monodromy at infinity and Fourier transform. II », Publ. Res. Inst. Math. Sci. 42 (2006), p. 803-835.

[38] M. Saito - « Mixed Hodge modules», Publ. Res. Inst. Math. Sci. 26 (1990), p. 221-333.

[39] D. Siersma \& M. TiBĂR - « Singularities at infinity and their vanishing cycles », Duke Math. J. 80 (1995), p. 771-783.

[40] J. Steenbrink \& S. ZuckeR - « Variation of mixed Hodge structure. I », Invent. Math. 80 (1985), p. 489-542.

[41] M. TIBĂR - «Asymptotic equisingularity and topology of complex hypersurfaces », Int. Math. Res. Not. 1998 (1998), p. 979-990.

[42] _ Polynomials and vanishing cycles, Cambridge Tracts in Mathematics, vol. 170, Cambridge Univ. Press, 2007.

[43] A. N. VARČENKO - « Theorems on the topological equisingularity of families of algebraic varieties and families of polynomial mappings », Izv. Akad. Nauk SSSR Ser. Mat. 36 (1972), p. 957-1019.

[44] J.-L. Verdier - «Stratifications de Whitney et théorème de BertiniSard », Invent. Math. 36 (1976), p. 295-312.

[45] H. H. Vui \& L. D. TRÁNG - « Sur la topologie des polynômes complexes », Acta Math. Vietnam. 9 (1984), p. 21-32 (1985).

[46] A. ZAHARIA - «On the bifurcation set of a polynomial function and Newton boundary. II », Kodai Math. J. 19 (1996), p. 218-233. 